\title{
(
}

UNIVERSIDAD PERUANA DE CIENCIAS APLICADAS

FACULTAD DE INGENIERÍA

CARRERA DE INGENIERÍA CIVIL

\section{Análisis y diseño estructural de un tanque elevado de concreto armado con capacidad de $2000 \mathrm{m3}$}

Tesis

para optar el Título Profesional de Ingeniero Civil

Autor:

Coral Carrera, Kevin Mijail (0000-0003-1899-5352)

Fernández Arana, Shelly Beatriz (0000-0003-3262-6377)

Asesor:

Ing. Victor Fernandez Dávila (0000-0002-1333-8989)

Lima, Agosto de 2018 
DEDICATORIA

Esta Tesis se la dedicamos a nuestros padres por su apoyo incondicional, ya que ellos son el principal cimiento en la construcción de nuestra vida profesional. A nuestra alma mater por incentivarnos las buenas prácticas en nuestro rubro ya que nos guiaron para desarrollarnos de manera óptima en esta carrera. 


\section{AGRADECIMIENTOS}

Agradecemos al Ing. Víctor Fernández Dávila, asesor de nuestra tesis, por su inestimable ayuda y paciencia desde los primeros pasos en la elaboración de esta tesis. Sus aportaciones han sido de mucha importancia, hemos podido contar con un asesor que ha sabido transmitirnos su experiencia y profesionalismo en el campo de la ingeniería estructural.

Un agradecimiento de manera especial al Ing. Jhojan Castaño, por habernos brindado la oportunidad de recurrir a su capacidad, experiencia y conocimiento científico, así como también haber tenido la paciencia de realizar las críticas constructivas a la tesis. 


\section{RESUMEN}

Los tanques elevados para el almacenamiento de agua desempeñan un rol muy importante en los sistemas de distribución de agua. Es importante realizar un adecuado análisis y diseño sismoresistente debido a que el Perú se encuentra en una zona sísmica por la interacción de la placa de Nazca y la Sudamericana.

En el Perú no se cuenta con normas necesarias ni específicas para el correcto análisis y diseño de este tipo de estructuras no convencionales. Por lo cual, además de la Norma de Diseño Sismoresistente (E.030) y Diseño de Concreto Armado (E.060) del Reglamento Nacional de Edificaciones, se usaron las Normas Internacionales Seismic Design of Liquid - Containing Concrete Structures and Commentary (ACI 350.3 - 06) y American Society of Civil Engineers (ASCE 7-05), así mismo, para el modelo dinámico de interacción del líquido con la estructura se usó el modelo propuesto por G.W.Housner. En este modelo la masa total del líquido se clasifica en Masa Impulsiva y Masa Convectiva. Para el modelamiento se usó el programa de elementos finitos SAP2000 Versión 18.

Para el análisis dinámico se usó el mapa de isoaceleraciones espectrales con el cual se calculó un espectro de respuesta sísmico dando como resultado un periodo de $\mathrm{T}=4.01 \mathrm{~s}$ y una cortante de $V=1496.68$ Ton-f. Para fines de cálculo se consideró la ubicación en la ciudad de Lima y el tipo de suelo rígido.

Finalmente, se propone el uso de anillos deflectores en la cuba, las cuales estarán adosadas en la pared interna en forma de viga. Esta propuesta es un sistema de amortiguamiento sísmico que disminuye las fuerzas producidas por la masa del agua hasta en un 30\%. La idealización de la propuesta, cálculos y resultados se verán con mayor detalle en la presente tesis.

Palabras clave: Diseño sísmico; Estructuras; Dispositivo antioleaje; Efecto Pedelta; Modelamiento estructural de tanque. 


\section{ABSTRACT}

Elevated water storage tanks constitute an important role in water distribution systems. It is important to conduct a proper analysis and earthquake resistant design, because Peru is in a seismic zone caused by the interaction of the Nazca plate and the South American plate.

In Peru there are no adequate nor specific norms for the correct analysis and design of this type of unconventional structures. Therefore, in addition to the Sismo Resistant Design Standard (E.030) and Armed Concrete Design (E.060) of the National Building Regulations, the International Standards Seismic Design of Liquid Concrete Structures and Comments (ACI 350.3 - 06) and American Society of Civil Engineers (ASCE 7-05), likewise, for the dynamic model of interaction of the liquid with the structure the model proposed by GW Housner was used. In this model, the total mass of the liquid is classified as Impulsive Mass and Convective Mass. For modeling, the finite element program SAP2000 Version 18 is used.

For the dynamic analysis, the map of spectral isoacelerations was used, with which a seismic response spectrum was calculated, resulting in a period of $\mathrm{T}=4.01 \mathrm{~s}$ and a shear of $\mathrm{V}=1496.68$ Ton-f. For the purposes of calculation, the location in the city of Lima and the type of rigid floor.

Finally, it is proposed to use baffle rings in the tank, which are attached to the internal wall in the form of a beam. This proposal is a system of seismic damping that reduces the forces produced by the mass of water by up to $30 \%$. The idealization of the proposal, calculations and results will be seen in greater detail in this thesis.

Keywords: Seismic design; Structures; Anti-collapse device; Pedelta effect; Structural tank modeling 


\section{Indice de contenido}

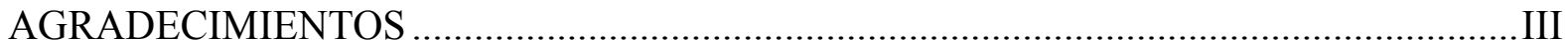

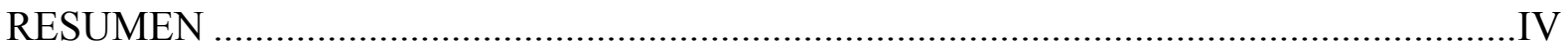

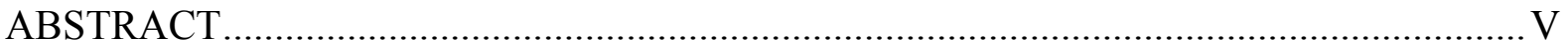

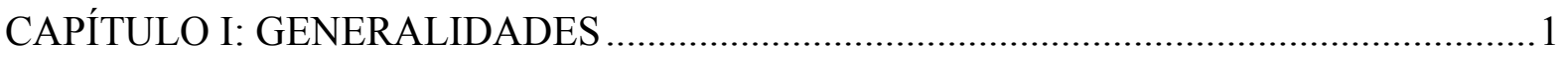

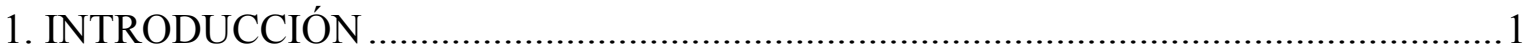

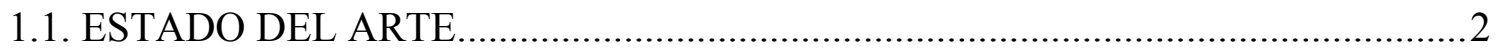

1.2. PLANTEAMIENTO Y FORMULACIÓN DEL PROBLEMA .................................

1.3. OBJETIVOS DE LA INVESTIGACIÓN …..........................................................

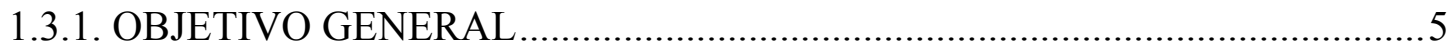

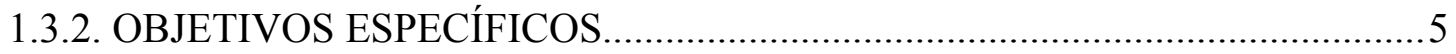

1.4. JUSTIFICACIÓN DE LA INVESTIGACIÓN ...........................................................

1.5. IMPORTANCIA DEL SISTEMA ESTRUCTURAL ............................................

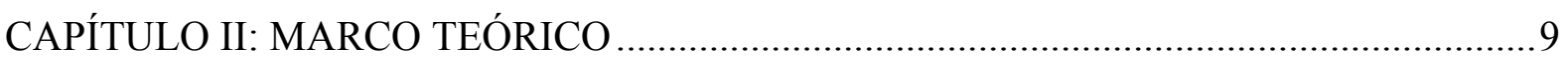

2.1. ELEMENTOS DEL SISTEMA ESTRUCTURAL ..............................................

2.2. DEFINICIÓN DEL SISTEMA MECÁNICO EQUIVALENTE .............................11

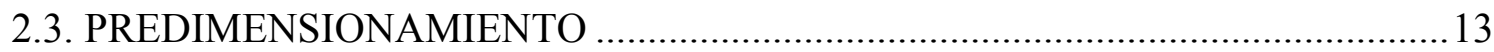

2.3.1. CÁLCULO DE DIMENSIONES PRINCIPALES ........................................... 14

2.4. PARÁMETROS PARA EL DISEÑO SEGÚN LA NORMA ACI 350 .................... 19

2.5. CRITERIOS GENERALES DE ESTRUCTURACIÓN .........................................26

2.5.1. CÁLCULO DE MASAS Y PESO DE LA ESTRUCTURA ..............................26

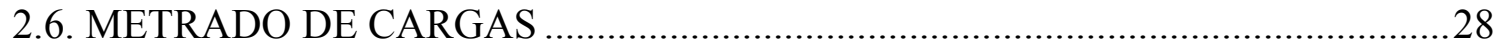

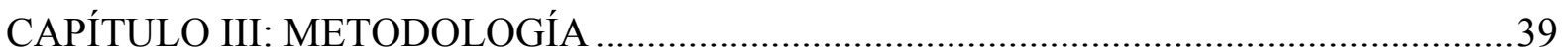

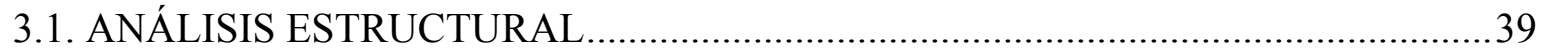

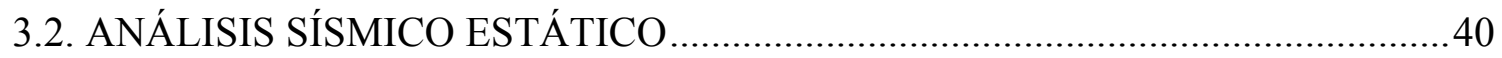

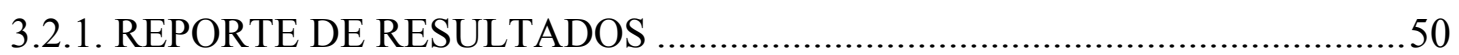

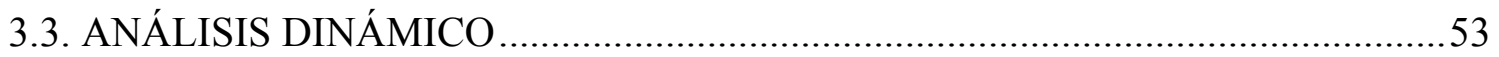

3.3.1. Espectro Dinámico con Factor de Escala..........................................................59

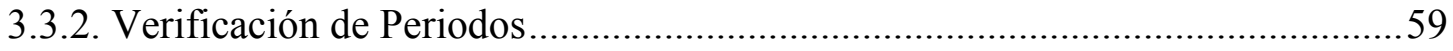

3.3.3. Verificación de Desplazamientos ......................................................................62 


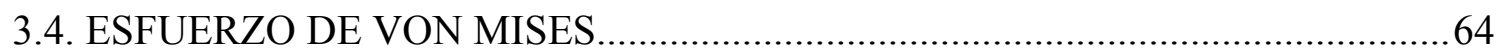

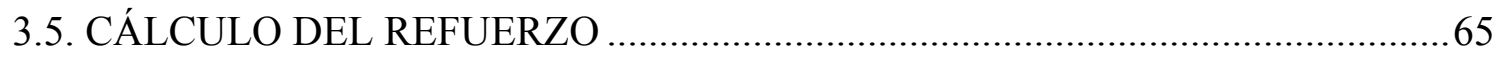

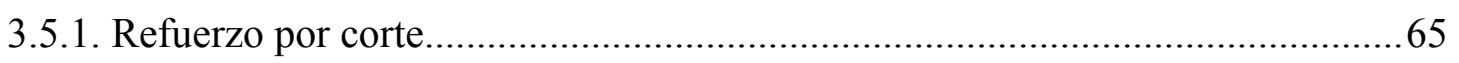

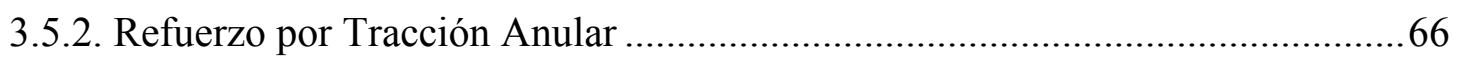

3.5.3. Refuerzo por Compresión en muros de carga...................................................67

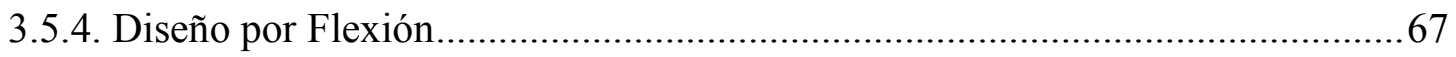

3.5.5. Espaciamiento Máximo Por Fisuramiento ........................................................69

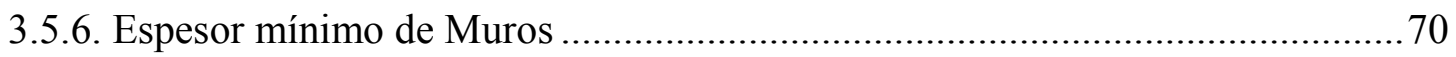

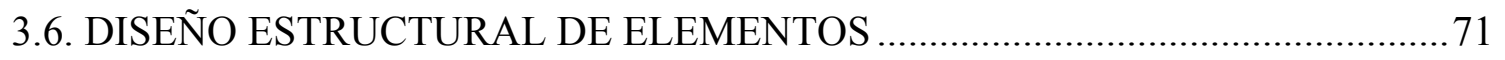

3.6.1. DISEÑO DE CÚPULA SUPERIOR ….......................................................... 73

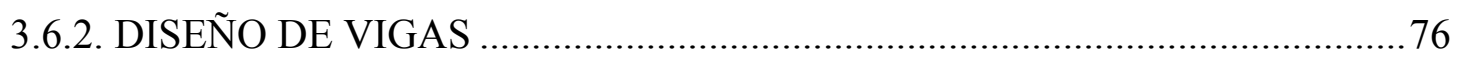

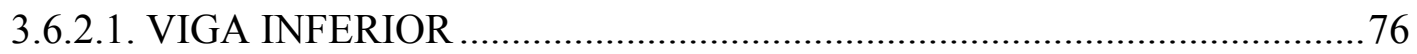

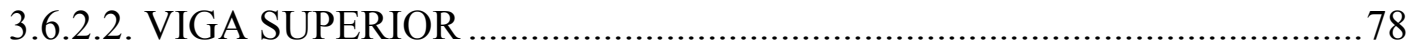

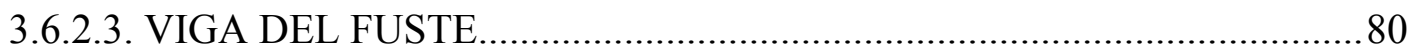

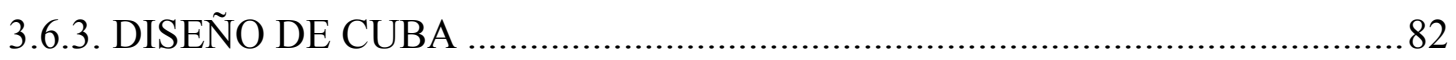

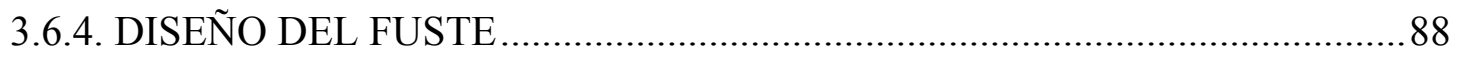

3.6.5. DISEÑO DE FONDO TRONCOCÓNICO .......................................................94

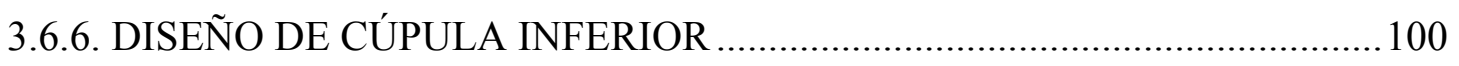

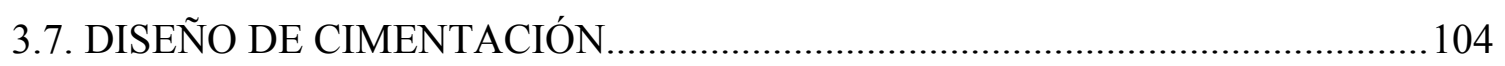

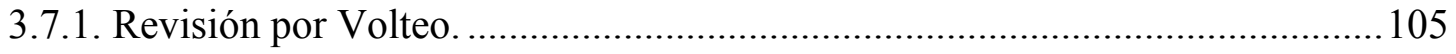

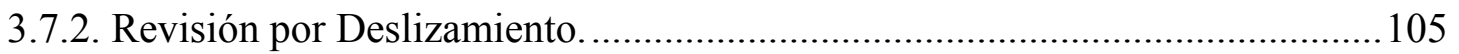

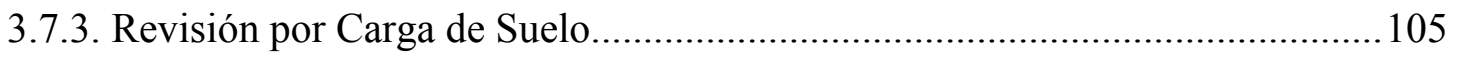

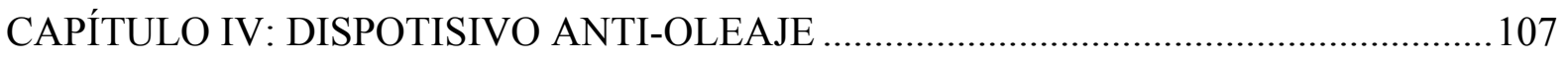

4.1. DISPOSITIVO ANTI-SLOSHING PARA LA ESTRUCTURA ............................... 107

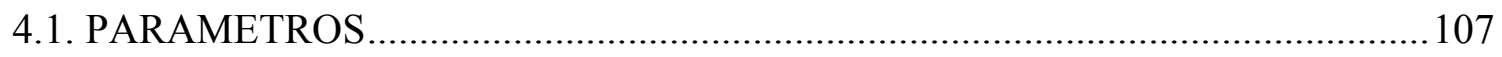

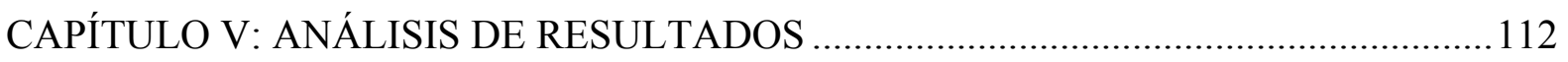

5.1. ANÁLISIS DE RESULTADOS CON EL USO DE ANILLOS DEFLECTORES ...112

5.1.1. COMPARACIÓN DE FUERZAS ACTUANTES EN LA ESTRUCTURA .......112

5.1.2. COMPARACÍON DE DESPLAZAMIENTOS ............................................... 113

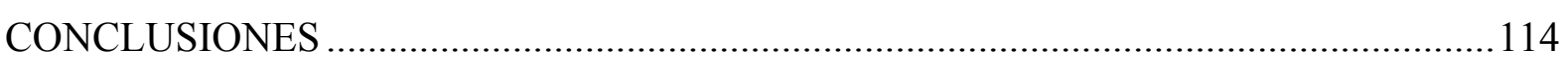

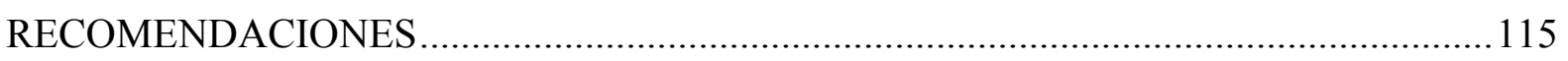

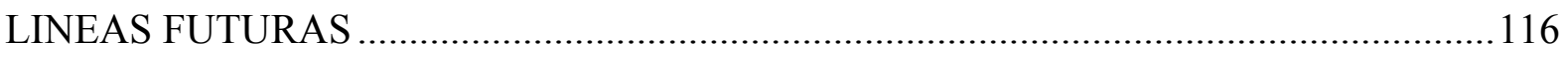




\section{Índice de Imágenes}

Figura 1-I: Cantidad de reservorios por capacidad ........................................................

Figura 2-II: Elementos Estructurales del Tanque Elevado. ................................................ 10

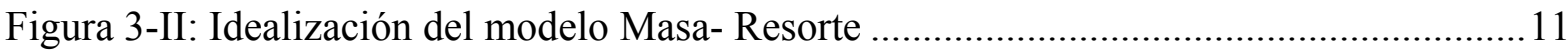

Figura 4-II: Sistema Mecánico Equivalente de G.W. Housner ............................................ 12

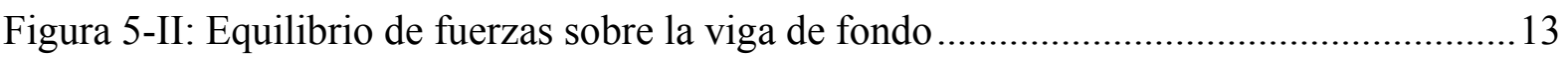

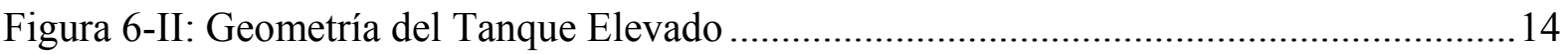

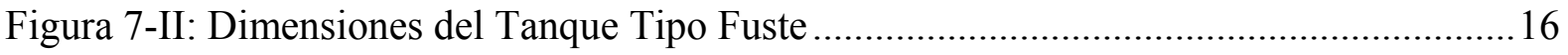

Figura 8-II: Alturas efectivas, convectivas e impulsivas.................................................. 17

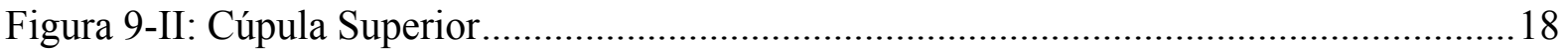

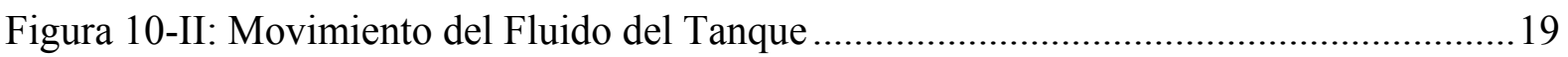

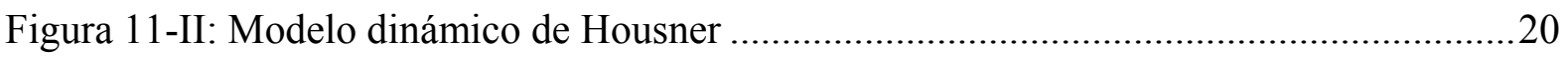

Figura 12-II: Razón de Masa Impulsiva y Convectiva vs D/HL..........................................23

Figura 13-II: Razón de altura de masa impulsiva y Convectiva vs D/HL.............................23

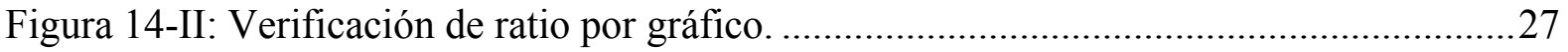

Figura 15-II: Verificación de he/HLy hi/HL por gráfico.....................................................28

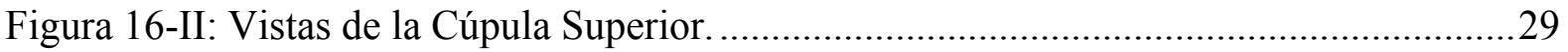

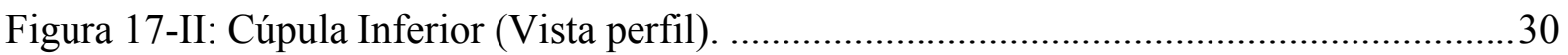

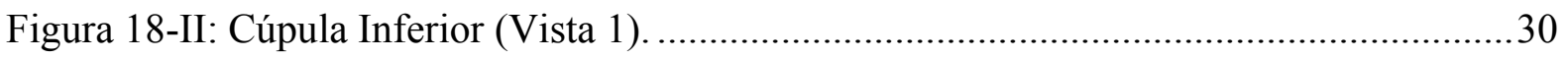

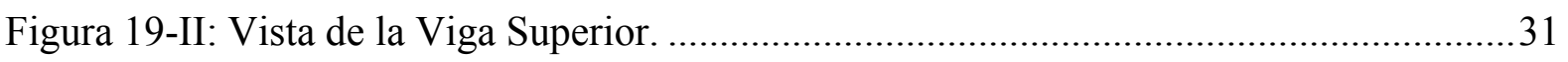

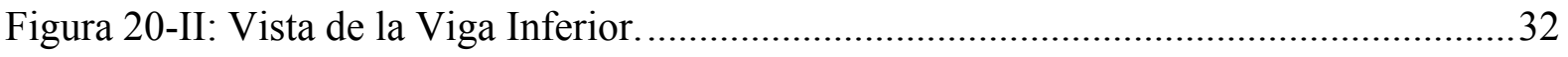

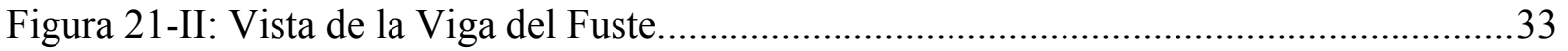

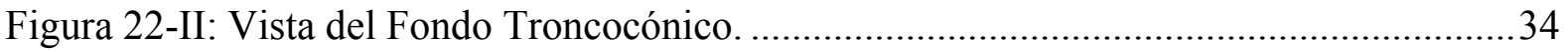

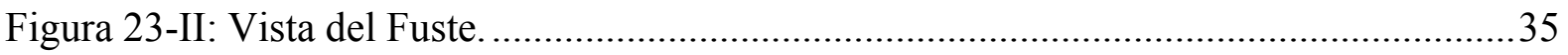

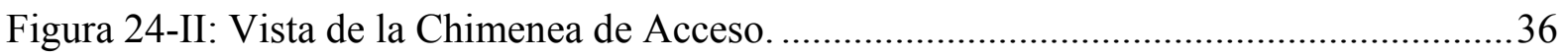

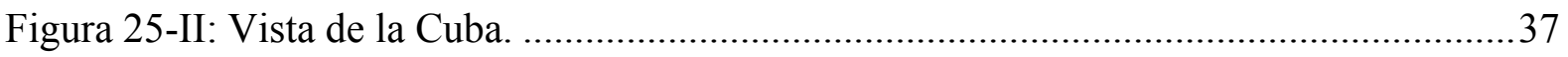

Figura 26-III: Cargas hidráulicas en la cuba .................................................................... 40

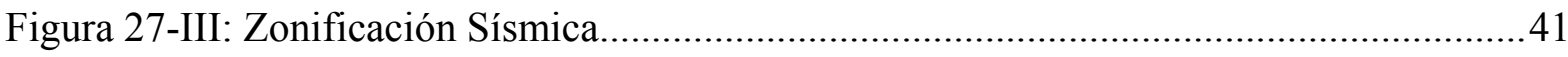

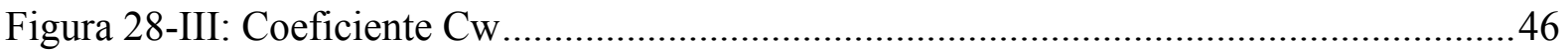

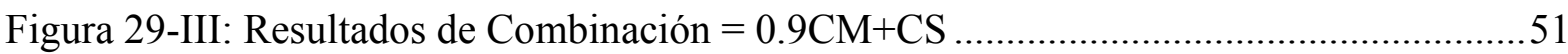




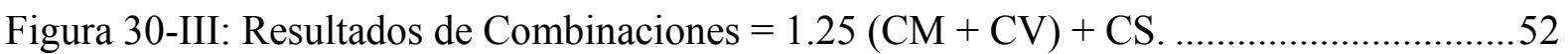

Figura 31 - III: Aceleración espectral debido a los parámetros sísmicos de la estructura.......56

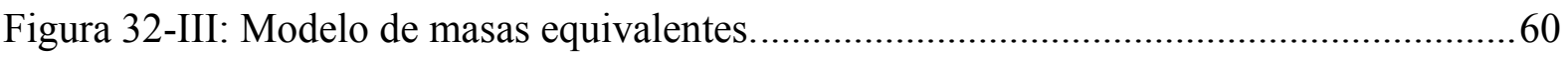

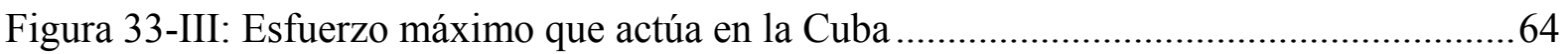

Figura 34: Deformación del acero $\square$ t para flexión positiva......................................................68

Figura 35: Área efectiva de la zona a tracción...................................................................... 70

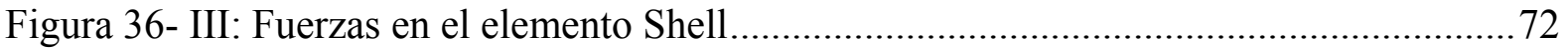

Figura 37- III: Momentos en el elemento Shell ..................................................................... 72

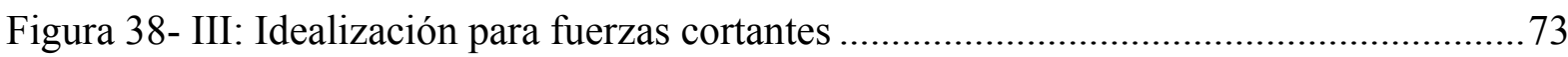

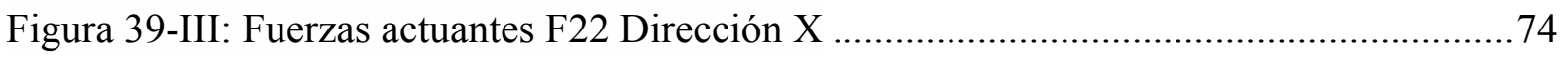

Figura 40-III: Fuerzas actuantes F11 Dirección "Y" .............................................................. 74

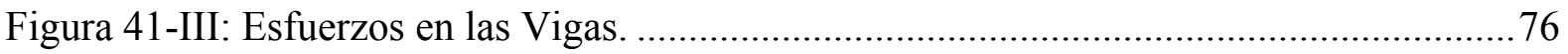

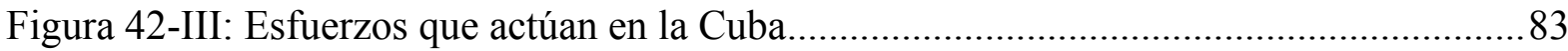

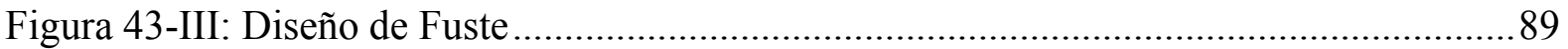

Figura 44-III: Esfuerzos en Fondo Troncocónico....................................................................94

Figura 45 - IV: cálculo del coeficiente C2 en función de la altura del tanque y el radio. ......109

Figura 46-IV: Modelo de rigideces y deflectores que influyen en la masa del fluido...........110

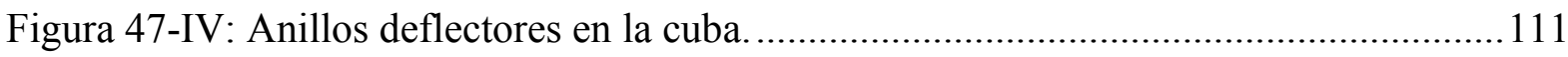




\section{Índice de Tablas}

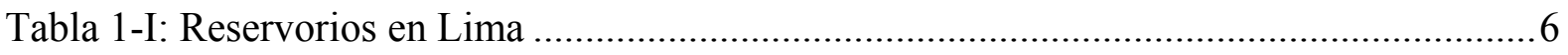

Tabla 2-I: Evaluación de reservorios tras el sismo en Pisco.................................................

Tabla 3-II: Resultados del dimensionamiento del tanque elevado ....................................... 16

Tabla 4-II: Tabla de Coeficientes de Reducción...................................................................25

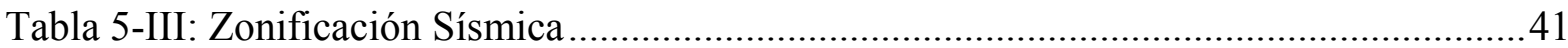

Tabla 6-III: Factor "S" suelo para determinación de parámetros sísmicos .............................42

Tabla 7-III: Parámetros para la determinación de periodos fundamentales. ...........................42

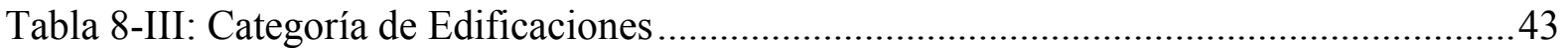

Tabla 9-III: Tabla de Coeficientes de Reducción. ....................................................................44

Tabla 10-III: Distribución de Fuerzas que actúan Sobre el Sistema Estructural. ....................49

Tabla 11-III: Resultados de desplazamientos máximos en el eje X (U2) para la combinación

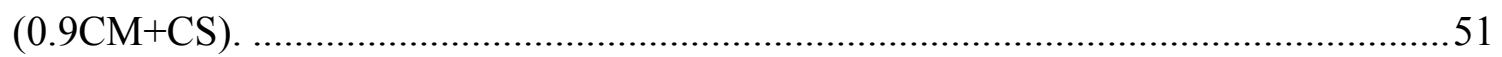

Tabla 12-III: Resultados de desplazamientos máximos en el eje X (U3) para la combinación

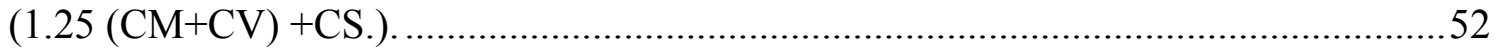

Tabla 13-III: Reporte de desplazamientos y análisis de resultados .....................................53

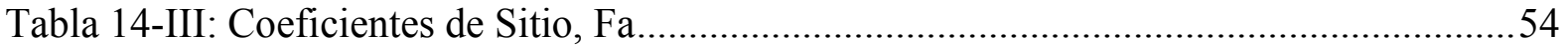

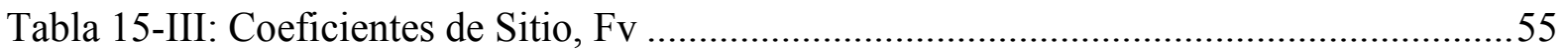

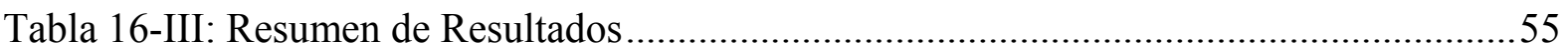

Tabla 17 - III: Cálculo de C para el espectro de respuesta ...................................................56

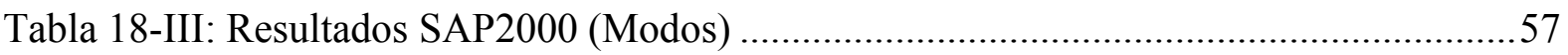

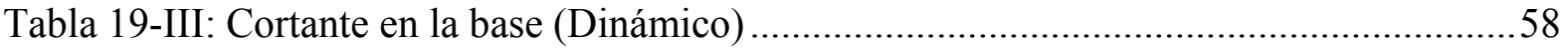

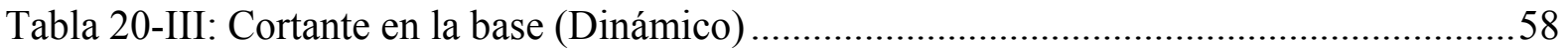

Tabla 21-III: Reporte de Desplazamientos para el estado Dinámico ......................................58

Tabla 22: Resultados SAP2000 (Modos) - Con Factor de Escala ...........................................59

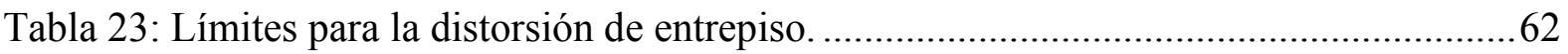

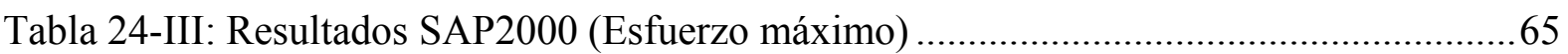

Tabla 25-III: Fuerzas que Actúan en La viga Inferior ....................................................... 76

Tabla 26-III: Fuerzas que actúan en la Viga Superior ............................................................ 79

Tabla 27-III: Fuerzas que actúan en la Viga del Fuste ........................................................... 80 
Tabla 28-III: Fuerzas y momentos que actúan sobre el elemento Cuba .83

Tabla 29: Fuerzas actuantes en el Fondo Troncocónico .......................................................95

Tabla 30-III: Fuerzas que Actúan en la Cúpula Inferior................................................... 100

Tabla 31-IV: Valores de Coeficiente Numérico "C" para la Estimación de Amortiguamiento

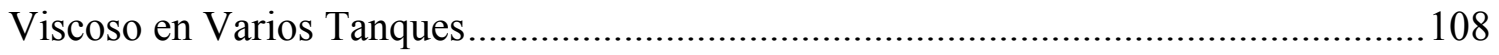

Tabla 32-IV: Comparación de Fuerzas Actuantes en la Estructura ......................................112

Tabla 33-IV: Comparación de desplazamientos para el análisis dinámico. ..........................113 


\section{CAPÍTULO I: GENERALIDADES}

\section{INTRODUCCIÓN}

La presente tesis tiene como objetivo realizar el análisis y diseño de un tanque elevado tipo intze de concreto armado para almacenamiento de agua. El aumento de la población y la necesidad de urbanizar nuevos espacios han dado lugar a proyectos de saneamiento, los tanques elevados para el almacenamiento de agua constituyen una parte muy importante en la red de distribución de agua, por ello es importante conocer el adecuado diseño y análisis de este sistema estructural hidráulico, tanto el diseño estático como el dinámico, cabe resaltar que el Perú se encuentra en una zona sísmica debido a la interacción de la placa de Nazca y la Sudamericana, se tienen antecedentes de sismos que han causado la destrucción total o parcial de este tipo de estructuras. Aunque se conoce la importancia de este tipo de estructuras, la normativa peruana no es suficiente para poder realizar un adecuado análisis y diseño, por lo cual también se hace uso de la normativa extranjera ACI. Es importante mencionar que los tanques son estructuras que requieren el uso de criterios de análisis sísmicos diferentes de aquellos utilizados en estructuras convencionales; debido a su comportamiento se necesita considerar además de los efectos inerciales impulsivos, los efectos hidrodinámicos convectivos sobre las paredes y el fondo de la cuba, para este análisis vamos a tomar como modelo a un sistema mecánico equivalente de G.W. Housner propuesto en 1963, el cual considera una masa fija y solidaria al movimiento de las paredes del tanque (masa impulsiva), y el resto de la masa se modela como una masa móvil unida al tanque por medio de resortes (masa convectiva). Para el modelamiento se va a hacer uso de un software llamado SAP2000, con la ayuda de esta herramienta computacional podremos interpretar los esfuerzos y realizar el diseño del tanque elevado de concreto armado. 


\subsection{ESTADO DEL ARTE}

Muchos investigadores han realizado estudios para el análisis del comportamiento hidrodinámico del agua almacenada en reservorios y tanques.

Las primeras investigaciones para el análisis de las ondas de los líquidos almacenados en contenedores, los cuales son sometidos a movimientos forzados fueron realizadas por Lamb en $1879 .^{1}$

Luego, en el año 1933 Westergaard determinó las presiones en un contenedor rectangular, cuando el contenedor está sometido a una aceleración horizontal. ${ }^{2}$

Graham y Rodríguez en el año 1952, consideran que las presiones dinámicas del líquido sobre las paredes del contenedor pueden separarse en dos masas; la masa convectiva y la masa impulsiva. ${ }^{3}$

G.W. Housner, en 1954 desarrolla en forma más detallada la teoría de las presiones sísmicas en los tanques de almacenamiento líquido y establece un modelo simplificado, en la parte media e inferior una componente llamada masa impulsiva, la cual está unida rígidamente a las paredes del contenedor; en la parte media y superior, la masa convectiva, la cual se une mediante resortes de rigidez “ $k$ " al contenedor y representa la acción del oleaje. En 1963 G.W.Housner desarrolla unas modificaciones en cuanto a las alturas en las cuales se ubican la masa convectiva e impulsiva. ${ }^{4}$

En 1974, Veltestos propuso un procedimiento para evaluar las fuerzas dinámicas en un contenedor, en el cual incorpora la flexibilidad de las paredes del tanque. ${ }^{5}$

${ }^{1}$ Cfr: Compagnoni, Curadelli y Martínez 2013: 2007

${ }^{2}$ Cfr: Housner 1954: 1

${ }^{3}$ Cfr: Flores 2009: 7

${ }^{4}$ Cfr: Compagnoni, Curadelli y Martínez 2013: 2007

${ }^{5}$ Cfr: Flores 2009: 7 
Método ASCE, propuesto por M.A. Haroun en 1997, este método al igual que los anteriores está basado en el modelo mecánico propuesto por Housner para tanques rígidos. El procedimiento considera dos modos de respuesta del líquido contenido en el recipiente, el impulsivo y el convectivo. El modelo mecánico considera tres masas que contribuyen a la respuesta sísmica de la estructura. ${ }^{6}$

Finalmente, de muchos de los estudios realizados se ha demostrado que la diferencia de los resultados obtenidos para tanques rígidos y flexibles no es sustancial, por lo cual se recomienda el uso de los parámetros que corresponden a los tanques con pared rígida para todos los tipos de tanques.

El modelo más usado para el diseño de tanques es el modelo analítico equivalente de G.W. Housner, la aproximación de este método no considera con exactitud la determinación del esfuerzo impulsivo, se considera un contenedor de paredes rígidas.

${ }^{6}$ Cfr: Haroum y Tayel 1985: 329 


\subsection{PLANTEAMIENTO Y FORMULACIÓN DEL PROBLEMA}

La Norma Peruana de Diseño Sismoresistente E.030 no contempla los factores necesarios para el correcto análisis de este tipo de estructuras. Se conoce que en el Perú son frecuentes y han causado daños importantes como los más recientes ocurridos en la región Sur (Arequipa y Moquegua) en el año 2001 y el sismo del 2007 en Pisco. ${ }^{7}$

Así mismo, para el análisis del tanque elevado se debe tomar en cuenta la interacción del líquido y la estructura que lo contiene, la consideración de cargas tales como, la carga muerta, carga viva, la carga del fluido y carga de sismo. Lo más relevante en este punto es el análisis sísmico-hidrodinámico.

Para llevar a cabo el análisis y diseño de este tanque elevado de concreto armado, se emplea el sistema mecánico equivalente propuesto por G.W Housner en el año de 1963, en el cual los efectos hidrodinámicos del agua se incluyen en el análisis con una masa que oscila dentro del tanque.

La idealización del sistema según Housner es interpretar la masa del fluido en dos estados una masa impulsiva (la parte inferior de la estructura) y otra masa convectiva (parte superior de la estructura). La masa impulsiva, se comporta y modela de manera muy rígida la cual genera, por la ley de Hooke, que las deformaciones del sistema sean mínimas y además que los periodos de oscilación, para este caso, sean menores. Por otra parte, la masa convectiva ocurre lo contrario ya que la rigidez es mucho menor y genera el oleaje (sloshing) la cual genera oscilaciones con periodos mayores, se modela como una masa móvil conectada a la estructura mediante resortes. ${ }^{8}$

\footnotetext{
${ }^{7}$ Cfr. Instituto Geofísico del Perú 2007:5

${ }^{8}$ Cfr: Rivera 1984: 20
} 


\subsection{OBJETIVOS DE LA INVESTIGACIÓN}

\subsubsection{OBJETIVO GENERAL}

Realizar el análisis y diseño estructural de un tanque elevado de concreto armado con capacidad de $2000 \mathrm{~m}^{3}$ para el almacenamiento de agua.

\subsubsection{OBJETIVOS ESPECÍFICOS}

- Realizar el predimensionamiento de los elementos estructurales del tanque elevado tipo intze.

- Realizar el análisis sísmico estático y dinámico, considerando para el análisis dinámico el modelo de G.W Housner.

- Realizar el análisis estructural haciendo uso de una herramienta computacional (SAP2000).

- Diseñar los elementos estructurales que conforman el tanque elevado de concreto armado para el almacenamiento de agua de acuerdo con la normativa peruana y extranjera vigente. Sólo se realizará el cálculo del acero de refuerzo para cada elemento estructural, ya que se está considerando un tanque de manera general. Para un proyecto específico se deberá realizar el análisis poblacional para hallar las dimensiones del tanque y se deberán actualizar los factores para el análisis y diseño, además del EMS para el diseño de cimentación.

- Realizar el análisis de un disipador que permita disminuir los efectos de la fuerza sísmica en la estructura.

\subsection{JUSTIFICACIÓN DE LA INVESTIGACIÓN}

El presente proyecto de investigación tiene diversas razones por las cuales es necesario que se desarrolle.

Primero, la tesis pretende ser un manual aplicativo para el análisis y diseño de un tanque elevado de concreto armado ya que las normativas peruanas no son suficientes para realizar dicho análisis, debido a esto nos basamos en la norma extranjera ACI y al Reglamento Nacional de Edificaciones presentándolas en este proyecto y orientándolas de acuerdo a los distintos requerimientos, con el objetivos de que futuros tesistas, estudiantes de pregrado o cualquier profesional de ingeniería pueda acceder a la información directa y específica concerniente al 
análisis y diseño de tanques elevados de concreto armado y a las cargas, esfuerzos a los que están sometidos, y analizar el comportamiento y detalles que sugieren los códigos anteriormente mencionados.

Segundo, debido al incremento de la población en el Perú y en consecuencia el incremento de los proyectos de saneamiento, dan lugar a que en zonas con terreno llano se requiera la construcción de tanques elevados de concreto armado para poder dotar de servicio de agua potable a estas nuevas urbanizaciones. Se sabe también que el Perú se encuentra en una zona sísmica y que por tanto el diseño de este tipo de estructuras debe asegurar el adecuado comportamiento ante las solicitaciones sísmicas.

Tercero. Como punto final, la investigación se aplicará a un ejemplo de diseño a partir de la cual se mostrarán las consideraciones para el análisis y diseño de un tanque elevado, el cual también servirá de mucho para profesionales involucrados en el área del cálculo estructural.

En la siguiente tabla se puede observar que debido al incremento poblacional y la necesidad de abastecimiento de agua da lugar a la construcción de nuevos reservorios elevados en la misma zona.

Tabla 1-I: Reservorios en Lima

\begin{tabular}{|l|l|c|c|}
\hline \multicolumn{1}{|c|}{ CÓDIGO } & \multicolumn{1}{|c|}{ UBICACIÓN } & ESTADO & CAPACIDAD (m3) \\
\hline RP-3 Los Cedros & A.H. Los Cedros de Ventanilla & Operativo & 700 \\
\hline RP-2 Los Cedros & A.H. Los Cedros de Ventanilla & Operativo & 700 \\
\hline R-3 Villa Los Reyes & A.H. Villa Los Reyes & Operativo & 650 \\
\hline R-1 Villa Los Reyes & A.H. Villa Los Reyes & Operativo & 600 \\
\hline R-3 Pachacutec & Mz. I-7 lote 13 Sector C Pachacutec & Operativo & 1300 \\
\hline R-7 Pachacutec & Mz. LL-11 A.H. 12 de Octubre & Operativo & 1300 \\
\hline R-6 Pachacutec & Mz. LL-11 A.H. 31 de Diciembre & Operativo & 600 \\
\hline RP-3 & A.H. Tupac Amaru & Operativo & 100 \\
\hline RP-4 & A.H. Tupac Amaru & Operativo & 100 \\
\hline CR-273 & A.H. Tupac Amaru & Operativo & 100 \\
\hline RP-1 & A.H. Tupac Amaru (CR-274) & Operativo & 400 \\
\hline CR-116 & Horacio Zevallos & Operativo & 600 \\
\hline CR-200 & Horacio Zevallos & Operativo & 100 \\
\hline R-2 & Horacio Zevallos & Operativo & 100 \\
\hline R-2' & Horacio Zevallos & Operativo & 100 \\
\hline R-4 & Horacio Zevallos & Operativo & 50 \\
\hline CR-199 & Horacio Zevallos & Operativo & 50 \\
\hline
\end{tabular}

Fuente: Sedapal 2017. 
En el siguiente gráfico se muestra las dimensiones de los tanques elevados en Lima, siendo los más comunes los reservorios para volúmenes de 100 a $480 \mathrm{~m}^{3}$, llegando hasta volúmenes de más de $3000 \mathrm{~m}^{3}$ con reservorios apoyados. Para el caso de reservorios elevados, los volúmenes más grandes de almacenamiento son los de $2000 \mathrm{~m}^{3}$ y $3000 \mathrm{~m}^{3}$.

Figura 1-I: Cantidad de reservorios por capacidad

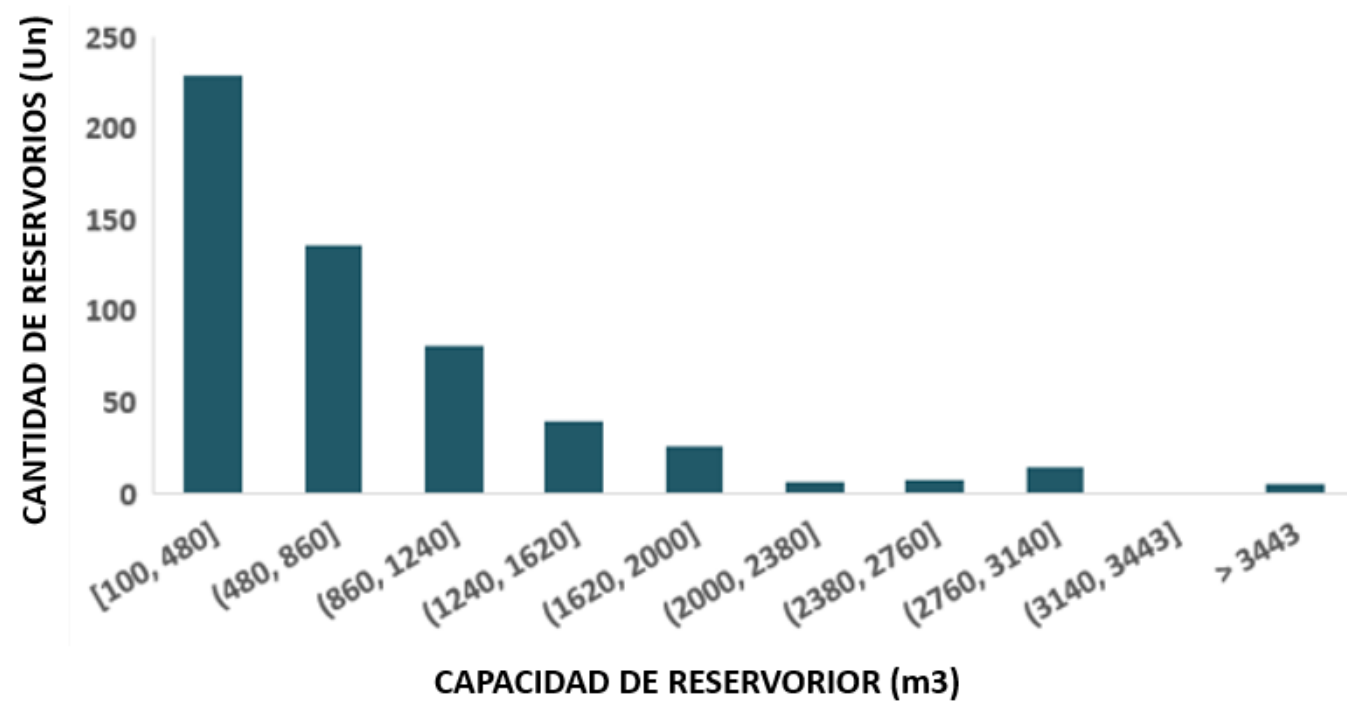

Fuente: Sedapal 2017. 


\subsection{IMPORTANCIA DEL SISTEMA ESTRUCTURAL}

Este sistema estructural de almacenamiento es una fundamental obra de arte hidráulica debido a que contempla la matriz principal de abastecimiento de agua potable a una población, por tanto, la construcción, el análisis y diseño de esta misma es esencial. Además, este sistema es de uso público, por lo cual el estudio de la demanda de la población debe ser de tal forma que esta pueda abastecer a toda esta localidad sin problemas de escasez de agua potable. ${ }^{9}$

Así mismo, es necesario diseñar esta estructura de manera que cumpla un desempeño óptimo, la cual incluya aspectos generales y términos que incorporen los comportamientos críticos que sean originadas por una eventualidad sísmica, teniendo como principal factor que el funcionamiento hidráulico del sistema y que la funcionalidad de esta no se vea afectada estructuralmente después del sismo, dado que al ser una estructura importante, es fundamental que esta se afecte de tal manera que la gestión de este recurso no sea un problema más cuando suceda una eventualidad sísmica. En la siguiente tabla se puede observar el estado de los reservorios después del sismo de Pisco ocurrido el 15 de agosto del 2007.

Tabla 2-I: Evaluación de reservorios tras el sismo en Pisco.

\begin{tabular}{|c|c|c|c|c|}
\hline ITEM & RESERVORIO & $\begin{array}{c}\text { CAPACIDAD } \\
(\mathbf{m} 3)\end{array}$ & ESTADO & TIPO \\
\hline 1 & ANGOSTURA LIMÓN & 300 & REGULAR & ELEVADO \\
\hline 2 & ANGOSTURA LIMÓN & 400 & REGULAR & APOYADO \\
\hline 3 & URB. SAN JOAQUÍN & 1000 & REGULAR & ELEVADO \\
\hline 4 & BALNEARIO HUACACHINA & 375 & MAL ESTADO & APOYADO \\
\hline 5 & URB. SAN ISIDRO & 350 & MAL ESTADO & ELEVADO \\
\hline 6 & URB. JOSÉ DE LA TORRE U. & 1500 & MAL ESTADO & ELEVADO \\
\hline 7 & ESTADIO JOSÉ PICASSO P. & 1500 & BUENO & ELEVADO \\
\hline 8 & AA.HH. ADICSA & 1000 & MAL ESTADO & APOYADO \\
\hline 9 & URB. ANGOSTURA ALTA & 600 & BUENO & ELEVADO \\
\hline 10 & CASERIO DE CACHICHE & 20 & MAL ESTADO & ELEVADO \\
\hline 11 & P.J. SANTA ROSA DE LIMA & 500 & REGULAR & APOYADO \\
\hline 12 & RESERVORIO CENTRAL & 1200 & MAL ESTADO & ELEVADO \\
\hline 13 & P.J. SEÑOR DE LUREN & 160 & REGULAR & APOYADO \\
\hline
\end{tabular}

Fuente: J. Kuroiwa 2012

\footnotetext{
${ }^{9}$ Cfr.: Hickey 2008: 67
} 


\section{CAPÍTULO II: MARCO TEÓRICO}

Para el análisis estructural estático y dinámico, a continuación, se presentarán los elementos que conforman la estructura, así como también la idealización de la estructura con el modelo mecánico equivalente planteado por G.W. Housner. Para realizar el análisis se tomará en consideración lo propuesto en la norma ACI-350 (Diseño Sísmico de Estructuras Contenedoras de Líquido) y la norma Peruana E 030 y E 060.

\subsection{ELEMENTOS DEL SISTEMA ESTRUCTURAL}

Los elementos estructurales que conforman el tanque elevado de concreto armado son:

Cúpula Esférica Superior: Es el elemento estructural que está ubicada en la parte superior del tanque elevado de concreto armado, este elemento tiene la forma de un casquete esférico. En este elemento se analizan los esfuerzos que producen el peso propio de la cúpula, este elemento no tiene contacto con el agua ni soporta grandes cargas.

Cúpula Esférica Inferior: A diferencia de la cúpula anterior, este elemento está en la base de la estructura contenedora de agua, por lo que está en interacción con el líquido.

Cuba: La cuba o recipiente que contiene el agua, tiene que estar diseñado para soportar las fuerzas de interacción con el agua (hidrostáticas e hidrodinámicas), sirve también para proteger al agua de la contaminación del ambiente externo. La cuba debe ser diseñada para resistir las fuerzas axiales, cortantes y momentos que se determinarán del análisis basado en la combinación de cargas.

Fuste Cilíndrico: Es la estructura de soporte de la cuba, el líquido y las cúpulas. El fuste está sometido a flexo compresión, el fuste tiene poca ductilidad ante un evento sísmico por ser un elemento esbelto y hueco. El fuste debe ser diseñado para proporcionar una resistencia suficiente, la conexión del fuste y la cuba (fondo troncocónico) se debe diseñar para resistir las fuerzas axiales, cortantes y momentos, los cuales serán hallados con el análisis de cargas.

Chimenea de Acceso: Es una pared cilíndrica interna con el diámetro necesario para la salida y llegada de tuberías, también para el acceso de una persona. 
Figura 2-II: Elementos Estructurales del Tanque Elevado.

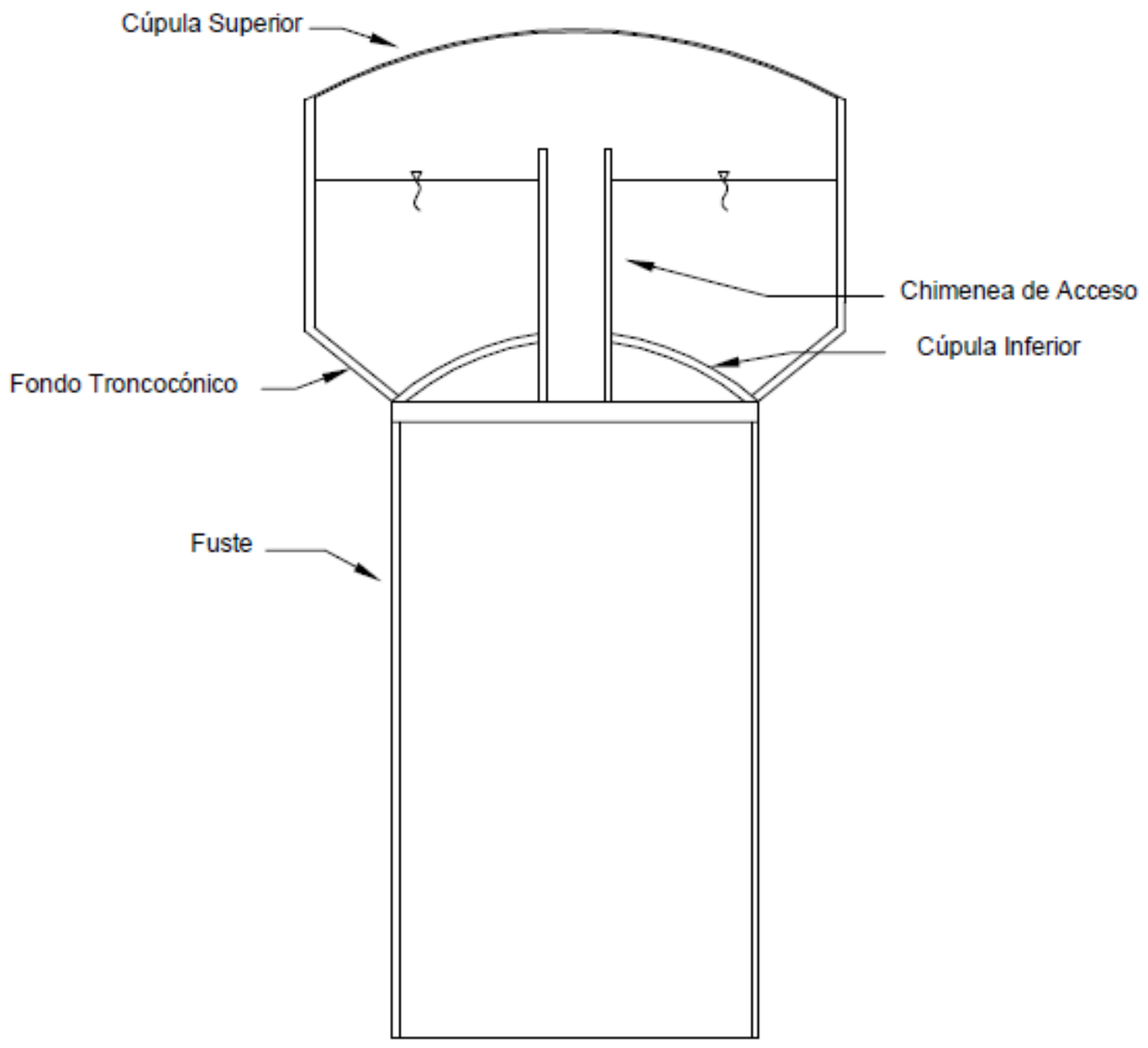

Fuente: Elaboración Propia.

En la figura 1-II; se muestra los elementos estructurales que conforman el tanque elevado. 


\subsection{DEFINICIÓN DEL SISTEMA MECÁNICO EQUIVALENTE}

El modelo usado para el desarrollo será el propuesto por G.W. Housner en 1963, el fluido es representado por una masa llamada masa impulsiva $m_{i}$ la cual se representa unida rígidamente a la pared del contenedor o cuba, y por otra masa llamada masa convectiva $m_{c}$ la cual se conecta a la pared de la cuba mediante resortes con una rigidez que se determinará " $k$ ". Se podría considerar una serie de masas convectivas las cuales simulan las frecuencias naturales del oleaje o también llamado sloshing, pero estas tienen una influencia despreciable para el análisis de las fuerzas ejercidas sobre el contenedor o cuba. Es por esto que para el diseño práctico del tanque elevado de concreto armado en esta tesis se usará el modelo simplificado, el cual permite una formulación matemática adecuada y de fácil entendimiento, y de esta manera poder obtener una respuesta. ${ }^{10}$

A continuación, en la figura 2-II se muestra la idealización de las masas convectivas-resorte y masa impulsivas.

Figura 3-II: Idealización del modelo Masa- Resorte

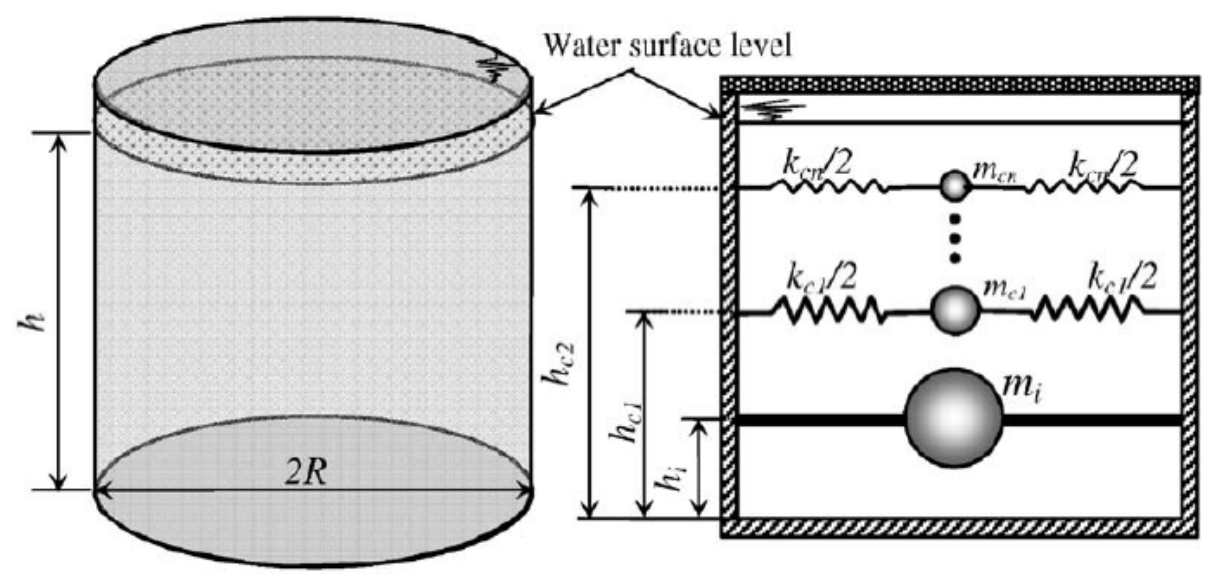

Fuente: "Simplified Seismic Analysis Procedures for elevated tanks considering fluidstructure - soil interaction”, R.Livaoglu y A.Donangün, p.423.

${ }^{10}$ Cfr: Compagnoni, Curadelli y Martínez 2013: 2009 
Figura 4-II: Sistema Mecánico Equivalente de G.W. Housner

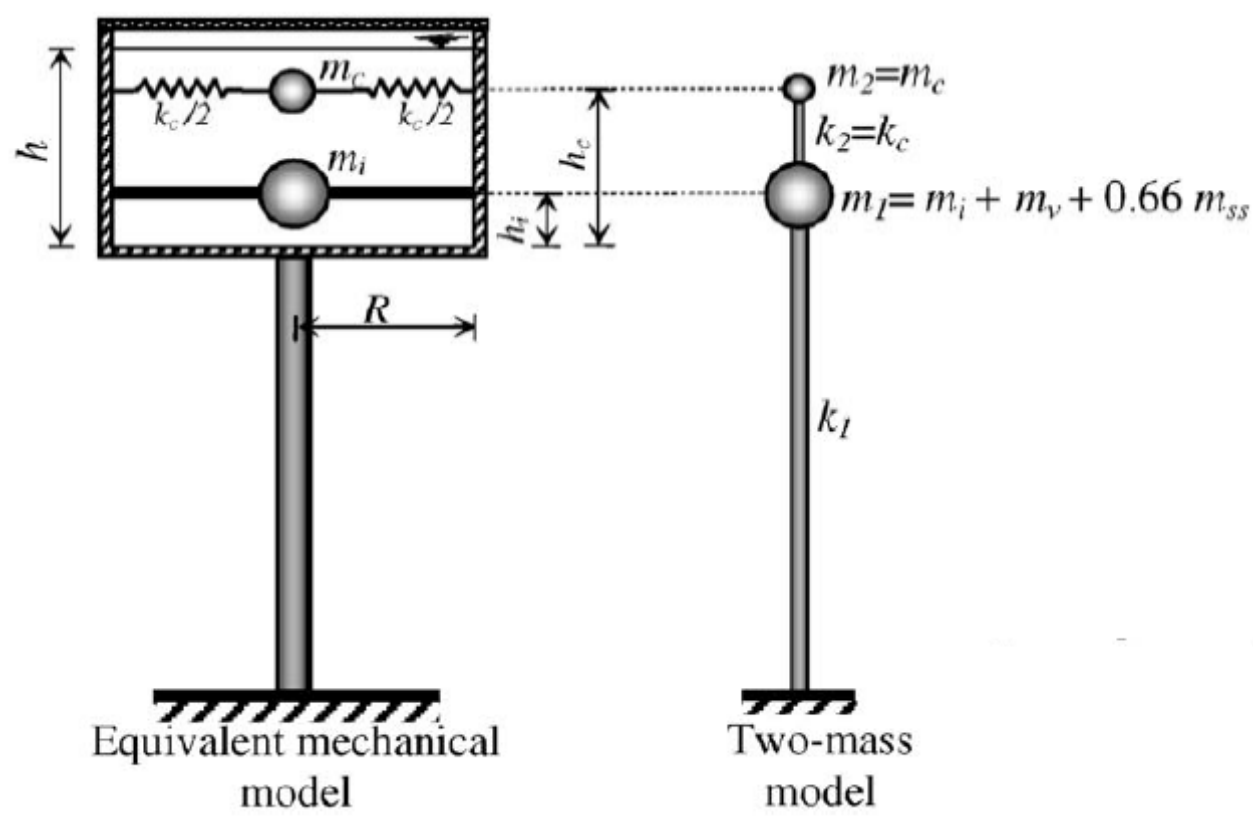

Fuente: "Simplified Seismic Analysis Procedures for elevated tanks considering fluidstructure - soil interaction”, R.Livaoglu y A.Donangün, p.424.

En la figura 3-II se muestra el sistema mecánico equivalente propuesto por G. W. Housner simplificado para el análisis.

Donde:

mv es la masa de la cuba vacía.

$\mathrm{m}_{\text {ss }}$ es la masa de la estructura portante (fuste).

$\mathrm{k} 1$ es la rigidez de la estructura portante.

$\mathrm{k}_{2}$ es igual a $\mathrm{k}_{\mathrm{c}}$ (rigidez de la masa convectiva).

$\mathrm{R}$ es radio de la cuba.

$h_{i}$ es la altura a la cual está ubicada la masa impulsiva.

mi es la masa impulsiva. 
$h_{c}$ es la altura a la cual está ubicada la masa convectiva.

$\mathrm{m}_{\mathrm{c}}$ es la masa convectiva.

h es la altura del líquido contenido.

La masa $\mathrm{m}_{1}$ constituye la masa impulsiva del líquido, la masa del peso de la cuba y una parte del peso de la estructura portante (fuste), el ACI 371R recomienda considerar los dos tercios del peso de la estructura portante. ${ }^{11}$

\subsection{PREDIMENSIONAMIENTO}

Se propuso como volumen de diseño tenemos 2,000 metros cúbicos, para la cual se realizará el predimensionamiento.

Los tanques elevados tipo INTZE se deben dimensionar de tal forma que los empujes de la viga circular de fondo que une la pared cónica con la esférica se anulen.

Figura 5-II: Equilibrio de fuerzas sobre la viga de fondo

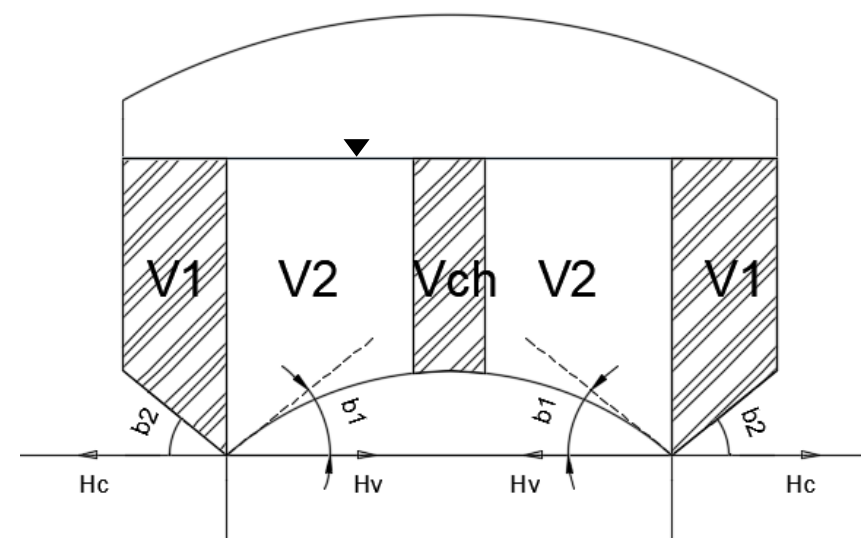

Fuente: Elaboración Propia

Las presiones Pc (Presión de la cúpula) y Pv (Presión del voladizo cónico) en toda la longitud $2 \pi \mathrm{b}$ es como la mostrada en la Ecuación 1:

${ }^{11}$ Cfr: .Livaoglu y Donangün 2006: 424 


$$
P c=\frac{\gamma \cdot V_{2}}{\operatorname{Sen}(b 1)} \quad ; \quad P v=\frac{\gamma \cdot V_{1}}{\operatorname{Sen}(b 2)}
$$

Así mismo, las componentes longitudinales serán como las mostradas en la ecuación 2:

$$
H c=\frac{\gamma \cdot V_{1}}{\operatorname{Tan}(b 2)} \quad ; \quad H v=\frac{\gamma \cdot V_{2}}{\operatorname{Tan}(b 1)}
$$

Teniendo en cuenta la condición de fondo para el reservorio tipo INTZE:

$$
\mathrm{Hc}=\mathrm{Hv}
$$

\subsubsection{CÁLCULO DE DIMENSIONES PRINCIPALES}

Figura 6-II: Geometría del Tanque Elevado

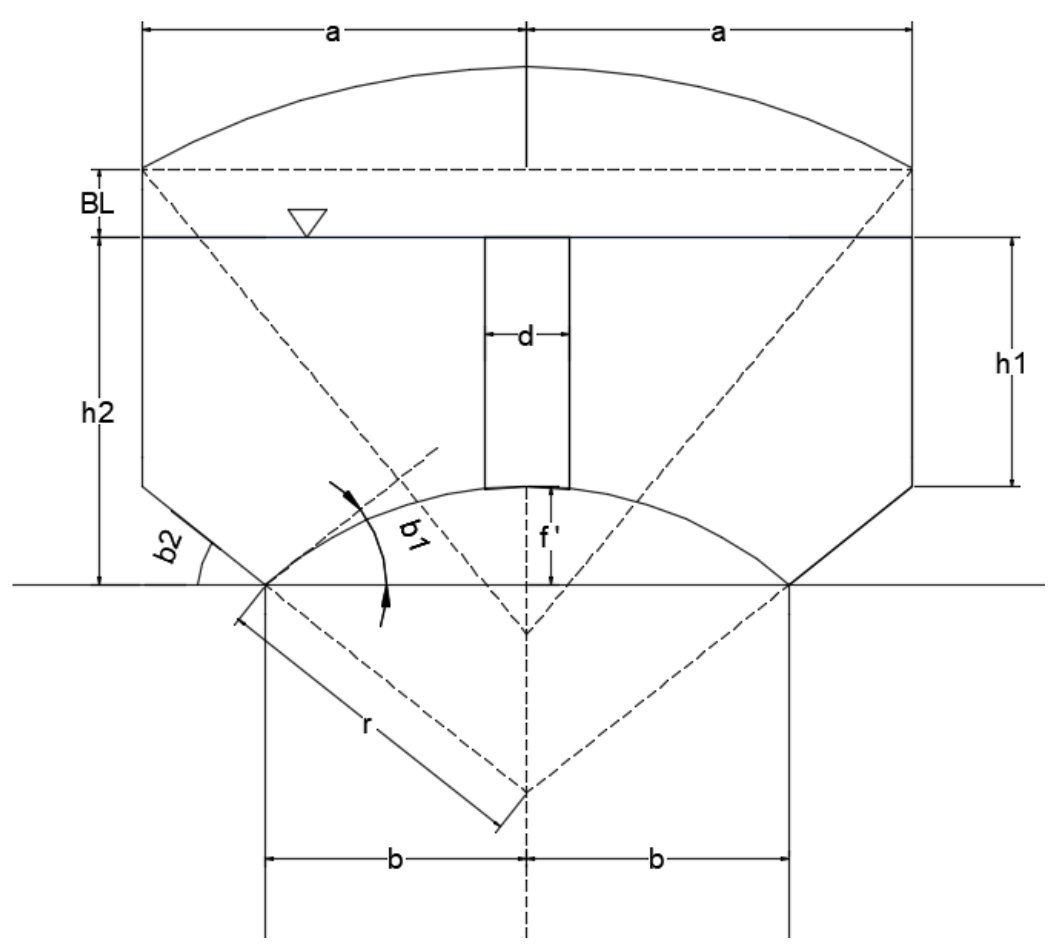

Fuente: Elaboración Propia

$$
\begin{gathered}
\mathrm{V}=\mathrm{V} 1+\mathrm{V} 2 \\
V_{1}=\left[\pi b^{2} h_{2}\right]-\left[\frac{\pi f^{\prime 2}\left(3 r * f^{\prime}\right)}{3}\right]-V_{c h}
\end{gathered}
$$




$$
V_{2}=\frac{(a-b)}{3} \pi\left[h_{1}(2 a+b)+h_{2}(2 b+a)\right]
$$

$$
V_{c h}=\pi d^{2} \frac{\left(h_{2}-f^{\prime}\right)}{4}
$$

$$
\begin{aligned}
& \tan (b 1)=\frac{b}{\sqrt{r^{2}+b^{2}}} \\
& \tan (b 2)=\frac{h_{2}-h_{1}}{a-b}
\end{aligned}
$$

Al ser un reservorio tipo INTZE, se deben anular las fuerzas sobre la viga inferior:

$$
\mathrm{Hv}=\mathrm{Hc}
$$

Considerando $\mathrm{b} 1=\mathrm{b} 2=45^{\circ}$

$$
V_{2}=V_{1}=\frac{V}{2}: r=b \sqrt{2} ; a-b=h_{2}-h_{1}
$$

Por el teorema de segmentos de cuerda en la cúpula tenemos:

$$
\begin{gathered}
2 r f^{\prime}=b^{2}+f^{\prime 2} \rightarrow f^{\prime}=b(\sqrt{2}-1) \\
V_{1}^{\prime}=\pi\left(b^{2}-d^{2}\right) h_{1} ; V_{2}^{\prime}=\pi\left(a^{2}-b^{2}\right) h_{1}
\end{gathered}
$$

Se obtiene:

$$
b=\sqrt{\frac{a^{2}+b^{2}}{2}}
$$

Considerando: $\mathrm{h} 1=\mathrm{a}$ y $\mathrm{h} 2=\mathrm{b}$ y reemplazando en la ecuación 4 .

$$
V_{2}=\frac{(a-b)}{3} \pi\left[a^{2}+b^{2}+4 a b\right]=\frac{V}{2}
$$

Resolviendo, se tienen los siguientes valores (aproximado para valores constructivos): 


$$
\begin{aligned}
& a=9.20 m \\
& b=6.25 m
\end{aligned}
$$

Considerando en el casquete de fondo:

$$
\text { 2. } R . f=a^{2}+f^{2} \rightarrow R=\frac{5}{3} a
$$

En base a lo cual, se muestra en la figura 6-II y tabla 1-II las medidas que se calcularon:

Figura 7-II: Dimensiones del Tanque Tipo Fuste

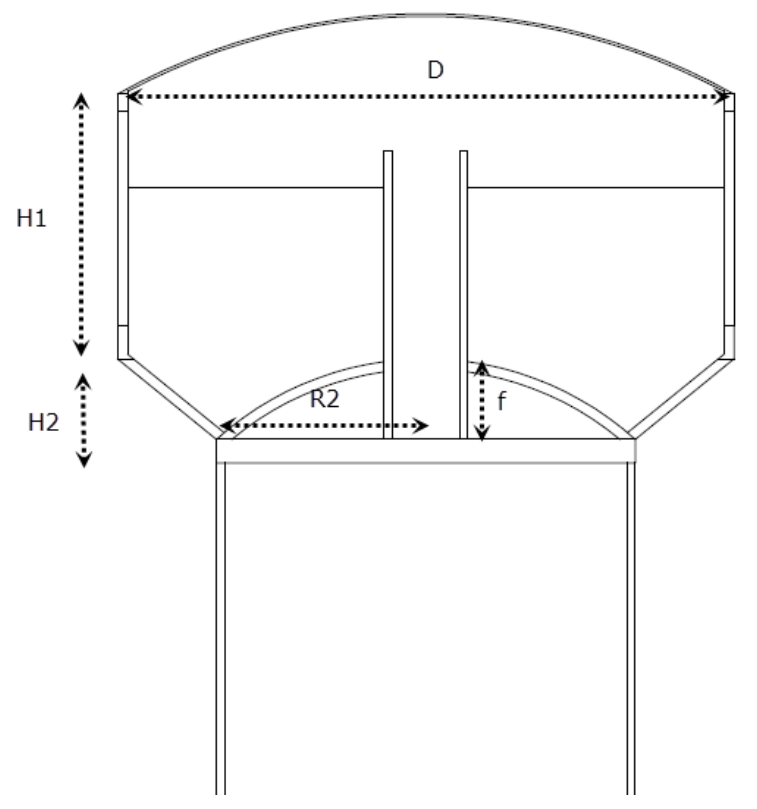

Fuente: Elaboración Propia

Tabla 3-II: Resultados del dimensionamiento del tanque elevado

\begin{tabular}{|ccc|}
\hline \multicolumn{3}{|c|}{ VOLUMEN DE } \\
ALMACENAMIENTO \\
\hline $\mathrm{D}=$ & 18.40 & $\mathrm{~m}$ \\
\hline $\mathrm{H} 1=$ & 6.36 & $\mathrm{~m}$ \\
\hline $\mathrm{H} 2=$ & 2.46 & $\mathrm{~m}$ \\
\hline $\mathrm{R} 2=$ & 6.25 & $\mathrm{~m}$ \\
\hline $\mathrm{f}=$ & 2.46 & $\mathrm{~m}$ \\
\hline $\mathrm{VT}=$ & 2000.00 & $\mathrm{~m} 3$ \\
\hline
\end{tabular}

Fuente: Elaboración Propia 
Así mismo, se tiene que hallar una altura efectiva (he), la cual es 4 veces el volumen total (de diseño) dividido por “pi” y 4 veces el diámetro principal. Esta altura, nos ayudará a calcular las alturas de la masa convectiva e impulsiva. ${ }^{12}$

Se calcula la altura efectiva geométricamente según lo siguiente:

$$
H e=4 * \frac{V}{\pi * D^{2}}
$$

Figura 8-II: Alturas efectivas, convectivas e impulsivas.

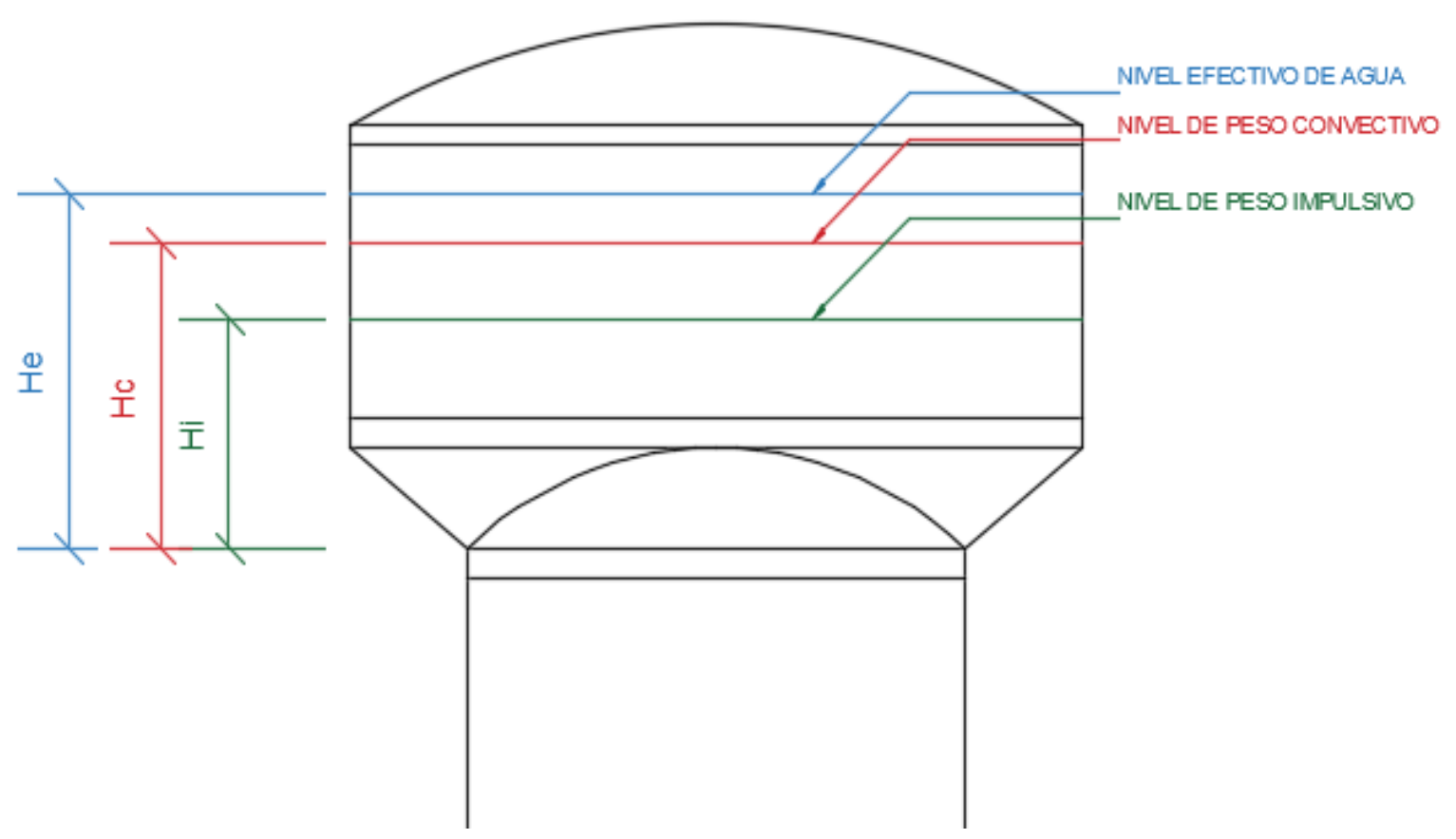

Fuente: Elaboración Propia.

$$
\mathrm{He}=7.68 \mathrm{~m}
$$

En la figura 7-II se muestra la idealización de las alturas en donde se encuentra las masas convectiva e impulsiva.

12 Organización Panamericana de Salud. (2005): 13 
Para este primer análisis se tomará tres principales elementos de la estructura, las cuales son, la cúpula, la cuba y el fuste. Las cuales según norma se predimensionan mediante los siguientes cálculos.

\section{Cúpula Superior}

En la figura 8-II se muestra la cúpula superior la cual se le asigna una altura aproximada.

Figura 9-II: Cúpula Superior

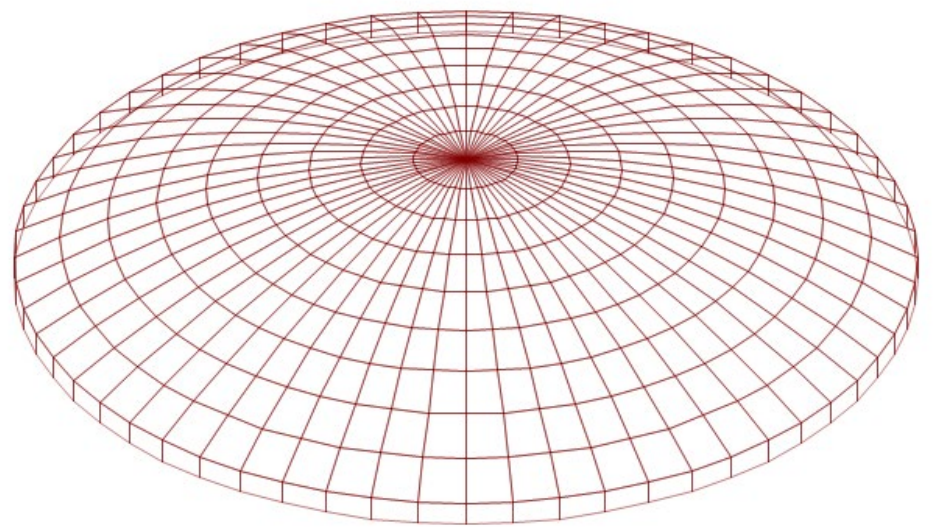

Fuente: Elaboración propia

$$
\mathrm{fe}=2.30 \mathrm{~m}
$$

Donde el "fe" será la altura aproximada que va desde el anillo superior hasta la parte superior de la cúpula.

\section{Pared Cilíndrica}

El espesor de la pared de la pared cilíndrica o Cuba, según norma esta debe ser calculada para un máximo de presiones hidrostáticas, las cuales son mayores a los dos tercios de altura efectiva (He), para nuestro caso se usó un ancho de $0.30 \mathrm{~m}$.

Fuste

Para la altura del fuste se considera una altura que proporcione la presión adecuada para la distribución de agua, será de $21 \mathrm{~m}$ de altura. El espesor se considera de $0.30 \mathrm{~m}$. 


\subsection{PARÁMETROS PARA EL DISEÑO SEGÚN LA NORMA ACI 350}

Este tanque elevado tipo intze se diseña con base al reglamento del American Concret Institute (ACI), la cual señala que esta estructura se debe comportar para las capacidades de resistencia y servicio, para esto se generan factores que aseguren ser fiables ante el colapso. Así mismo, en la norma se menciona el diseño sísmico teniendo como consideración las aceleraciones del suelo, características de la masa estructural, rigidez y amortiguamiento. Por lo tanto, esta norma brindará ciertos requisitos para analizar el comportamiento de la estructura ante cargas o aceleraciones. El ACI establece límites tolerables para la estructura y que estas sean adecuadas, así mismo, su comportamiento estructural no se altere para las distintas cargas que actúan en ella durante una eventualidad.

Housner desarrolló el modelo simplificado, la presión hidrodinámica del fluido convectivo causado por la oscilación del líquido en la parte superior y la presión del líquido impulsivo que se acelera con la estructura. La formulación matemática para tanques circulares de pared rígida (según ACI) como se muestra en la figura 9-II a continuación ${ }^{13}$ :

Figura 10-II: Movimiento del Fluido del Tanque

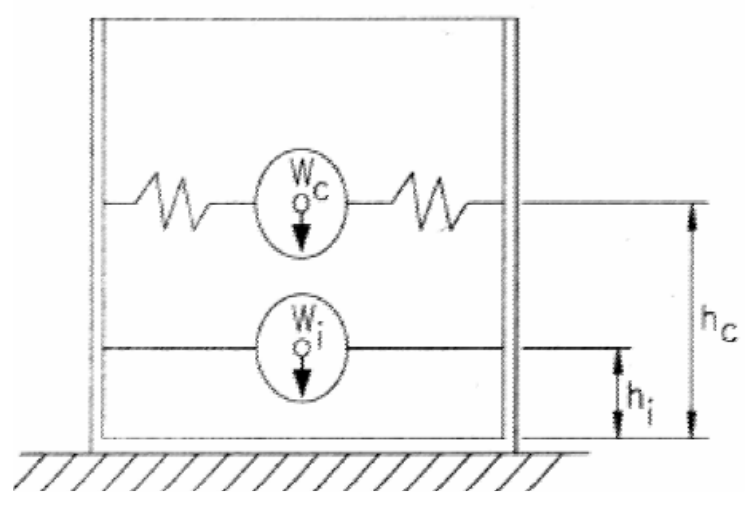

Fuente: "Seismic Design of Liquid Containing Concrete Structures" ACI 350.3. p43.

${ }^{13}$ Cfr: ACI 350.3 2008: 43 
El tanque al estar sometida a una aceleración horizontal provoca que la superficie genere oleaje o (sloshing), la cual puede alcanzar una altura máxima "dmáx" ${ }^{14}$ como se muestra en la figura 10-II a continuación.

Figura 11-II: Modelo dinámico de Housner

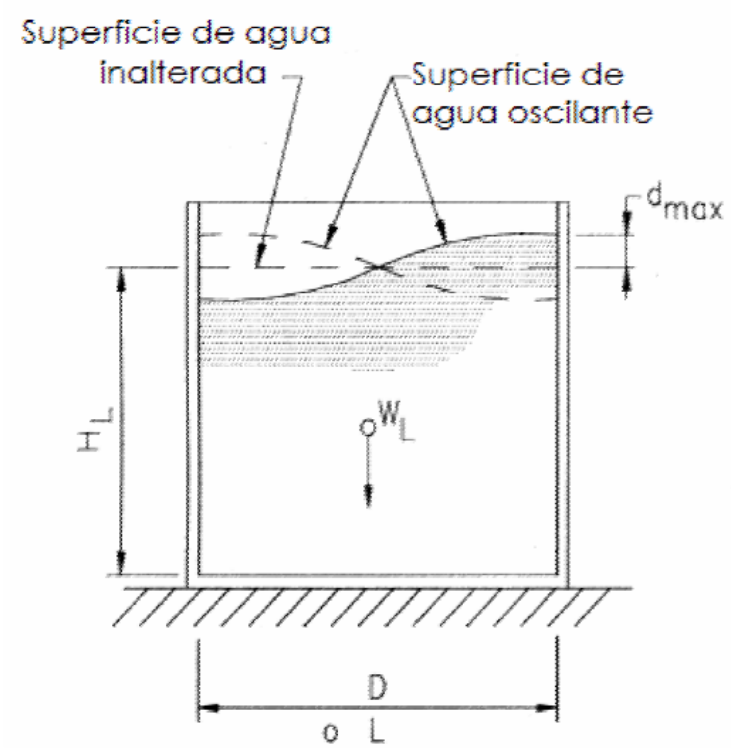

.Fuente: “Seismic Design of Liquid Containing Concrete Structures” ACI 350.3. p43.

Las características dinámicas de este modelo son estimadas usando las siguientes expresiones:

Frecuencia fundamental debido al Sloshing

La frecuencia fundamental producida por el oleaje de la masa convectiva se calcula de acuerdo a la Ecuación 11.

$$
\omega=\frac{1.84 \times g}{R} * \tanh \left(\frac{1.84 \times h}{R}\right)
$$

Donde:

g: aceleración de la gravedad.

R: Radio del recipiente contenedor del líquido.

${ }^{14}$ Cfr: ACI 350.3 2008: 43 
h: Altura del líquido.

Las masas para el modelo de G. W. Housner se calcularon mediante las Ecuaciones.

Masa Convectiva:

$$
\mathrm{mc}=\mathrm{m}_{\mathrm{w}} \mathrm{x} 0.318 \mathrm{x} \frac{\mathrm{R}}{\mathrm{h}} \mathrm{xtanh}\left(\frac{1.84 \mathrm{xh}}{\mathrm{R}}\right)
$$

Donde:

$\mathrm{m}_{\mathrm{w}}$ : Masa total del líquido.

$\mathrm{m}_{\mathrm{c}}$ : Masa convectiva.

Masa Impulsiva:

$$
\mathrm{mi}=\mathrm{m}_{\mathrm{w}} \cdot \frac{\tanh \left(\frac{1.74 \mathrm{xR}}{\mathrm{h}}\right)}{\left(\frac{1.74 \mathrm{xR}}{\mathrm{h}}\right)}
$$

Donde:

$\mathrm{m}_{\mathrm{w}}$ : Masa total del líquido.

$\mathrm{m}_{\mathrm{i}}$ : Masa Impulsiva.

Rigidez del Resorte:

La masa convectiva se modela con resortes las cuales están unidas a las paredes del tanque, la rigidez de estos resortes está calculada mediante la Ecuación 14.

$$
K=45 *\left(\frac{W c}{W L}\right)^{2} *\left(\frac{H_{L}}{D}\right)^{2} *\left(0.5 \frac{W L}{H L}\right)
$$

Para el caso de tanques elevados el ACI tiene un capítulo especial la cual tienen las siguientes consideraciones.

Cálculo del peso de los muros del reservorio 
La influencia de las presiones del agua en las paredes de la estructura, en la cuba, la cual es la masa de agua que será afectada por un factor de corrección debido a que la masa impulsiva se mueve conjuntamente con las paredes del tanque. Este coeficiente " $\varepsilon$ " representa una relación entre la masa dinámica y la masa total del tanque por influencia del agua. ${ }^{15}$

$$
\varepsilon=\left[0.0151 x\left(\frac{D}{H_{L}}\right)-0.1908 x\left(\frac{D}{H_{L}}\right)+1.021\right] \leq 1.0
$$

Dónde:

D: diámetro interior del reservorio.

$\mathrm{H}_{\mathrm{L}}$ : altura máxima del agua almacenada en el reservorio.

Cálculo de los pesos del líquido almacenado

Wi y $\mathrm{W}_{\mathrm{c}}$ la relación de pesos y alturas de la masa impulsiva y convectiva mediante la tabla propuesta por el ACI, la cual se presenta en las figuras 11-II y 12-II. ${ }^{16}$

$$
\begin{gathered}
\frac{W_{i}}{W_{L}}=\frac{\tanh \left(0.966\left(\frac{D}{H_{L}}\right)\right.}{0.866\left(\frac{D}{H_{L}}\right)} \\
\frac{W_{C}}{W_{L}}=0.230\left(\frac{D}{H_{L}}\right) \tanh \left(3.68\left(\frac{H_{L}}{D}\right)\right)
\end{gathered}
$$

${ }^{15}$ Cfr: ACI 350.3 2008: 41

${ }^{16}$ Cfr: ACI 350.3 2008: 44 
Figura 12-II: Razón de Masa Impulsiva y Convectiva vs D/HL.

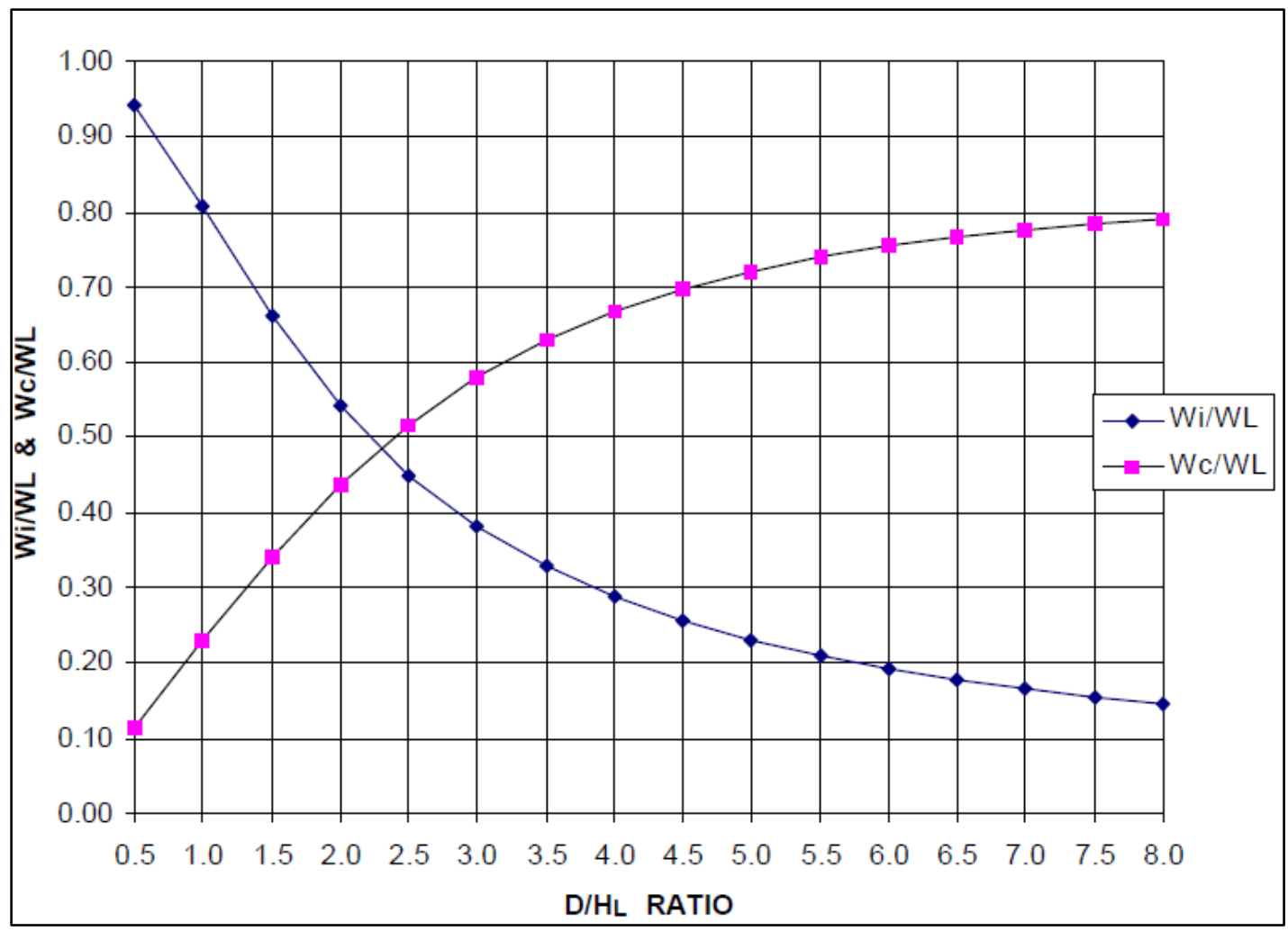

Fuente: "Seismic Design of Liquid Containing Concrete Structures” ACI 350.3. p44.

Figura 13-II: Razón de altura de masa impulsiva y Convectiva vs D/HL.

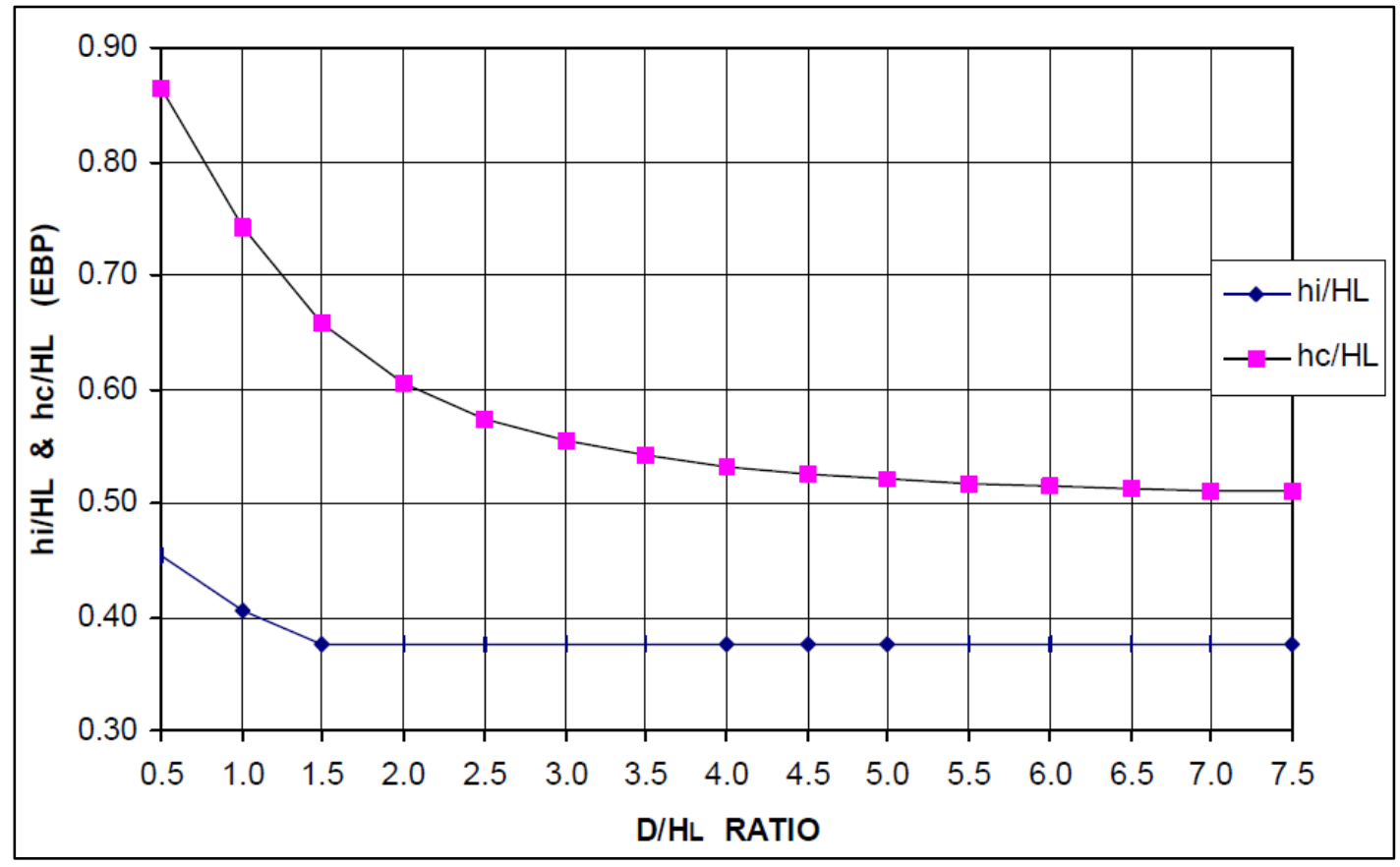

Fuente: "Seismic Design of Liquid Containing Concrete Structures" ACI 350.3. p45. 
Con las figuras anteriores (Figuras 11-II y 12-II), podemos obtener el centro de los pesos impulsivos y convectivo del líquido y las alturas en la que cada uno de estas masas actúa respectivamente.

También se puede hacer uso de las siguientes ecuaciones:

Para tanques con:

Diámetro menor a la altura de la cuba

$$
\begin{gathered}
\frac{D}{H_{L}}<1.333 \\
\frac{D}{H_{L}}<1.333 \longrightarrow \frac{h_{i}}{H_{L}}=0.5-0.09375\left(\frac{D}{H_{L}}\right)
\end{gathered}
$$

Para tanques con:

Diámetro mayor a la altura de la cuba

$$
\frac{D}{H_{L}} \geq 1.333 \quad \longrightarrow \frac{h_{i}}{H_{L}}=0.375
$$

En general:

$$
\frac{h_{C}}{H_{L}}=1-\frac{\cosh \left(3.68 \cdot\left(\frac{H_{L}}{D}\right)\right)-1}{3.68\left(\frac{H_{L}}{D}\right) \cdot \sinh \left(3.68 \cdot\left(\frac{H_{L}}{D}\right)\right)}
$$

Especificación de cargas sísmicas

Las cargas sísmicas se determinan de acuerdo a la norma del ACI la cual menciona que el factor de reducción ahora será afectado por este tipo de estructura la cual la llaman Factor de 
Modificación, no obstante, los coeficientes de uso, zona, suelo y amplificación sísmica serán tomados de la Norma E.030 de diseño sismo resistente.

Tabla 4-II: Tabla de Coeficientes de Reducción

\begin{tabular}{|l|c|c|c|}
\hline \multicolumn{1}{|c|}{ Tipo de estructura } & $\begin{array}{c}\mathbf{R} \\
\text { impulsiva }\end{array}$ & $\begin{array}{c}\mathbf{R} \\
\text { Enterrado }\end{array}$ & $\begin{array}{c}\mathbf{R} \\
\text { convectiva }\end{array}$ \\
\hline (a)Tanques anclados con base flexible & 3.25 & 3.25 & 1.0 \\
\hline $\begin{array}{l}\text { (b) Tanques monolíticos o empotrado en la } \\
\text { base }\end{array}$ & 2.0 & 3.0 & 1.0 \\
\hline (c) Tanques no anclados & 1.5 & 2.0 & 1.0 \\
\hline (d) Tanques elevados & $\mathbf{2}$ & - & 1.0 \\
\hline
\end{tabular}

Fuente: "Seismic Design of Liquid Containing Concrete Structures" ACI 350.3. p21.

Se realizarán los análisis sísmicos con diferentes factores de reducción, con la finalidad que tengamos valores más aproximados y la estructura no falle dado que para la masa convectiva la norma es más estricta. ${ }^{17}$

Determinación de la fuerza cortante total en la base

Esta fuerza se determina mediante las fuerzas que contemplan la estructura, la cual se calcula mediante la Ecuación 21. ${ }^{18}$

$$
V=\sqrt{(P i+P w+P r)^{2}+P c^{2}}
$$

Dónde:

Pi: Fuerza debido al peso impulsivo del liquido

Pw: Fuerza debido al peso de las paredes del reservorio

Pr: Fuerza debido al peso de la cúpula

Pc: Fuerza debido al peso convectivo del liquido

${ }^{17}$ Cfr: ACI 350.3 2008: 21

${ }^{18}$ Cfr: ACI 350.3R-01 2008: 17 


\subsection{CRITERIOS GENERALES DE ESTRUCTURACIÓN}

En este capítulo se calcularán todas las fuerzas estáticas, masa convectiva, masa impulsiva, altura de la masa convectiva, altura de la masa impulsiva, y factores que se presentan en la estructura y cómo estas influyen en el análisis sísmico estático y dinámico.

\subsubsection{CÁlCULO DE MASAS Y PESO DE LA ESTRUCTURA}

Los cálculos siguientes evaluados en hojas de cálculo Excel, nos ayudaran al análisis posterior del tanque elevado, ya que se modelará con estas respectivas masas y alturas que nos sugiere la norma y de acuerdo a esto diseñar la estructura que cumpla con los requisitos de las normas. ${ }^{19}$

Como se muestran en las siguientes formulas.

Cálculo del factor de corrección del peso del fluido

$$
\begin{aligned}
& \varepsilon=\left[0.0151 x\left(\frac{D}{H_{L}}\right)-0.1908 x\left(\frac{D}{H_{L}}\right)+1.021\right] \leq 1.0 \\
& \varepsilon=0.65 \\
& W w=\quad 3140188 \mathrm{Kgf}
\end{aligned}
$$

Cálculo de los pesos efectivos impulsivas y convectivas: ${ }^{20}$

$$
\begin{array}{r}
\frac{W i}{W_{L}}=\frac{\tanh \left(0.866 *\left(\frac{D}{H_{L}}\right)\right.}{0.866 *\left(\frac{D}{H_{L}}\right)} \\
\frac{W c}{W_{L}}=0.230 *\left(\frac{D}{H_{L}}\right) * \tanh \left(3.68 * \frac{H_{L}}{D}\right)
\end{array}
$$

${ }^{19}$ ACI 350.R3-1 (2008): 38

${ }^{20}$ ACI 350.R3-1 (2008): 44 
Figura 14-II: Verificación de ratio por gráfico.

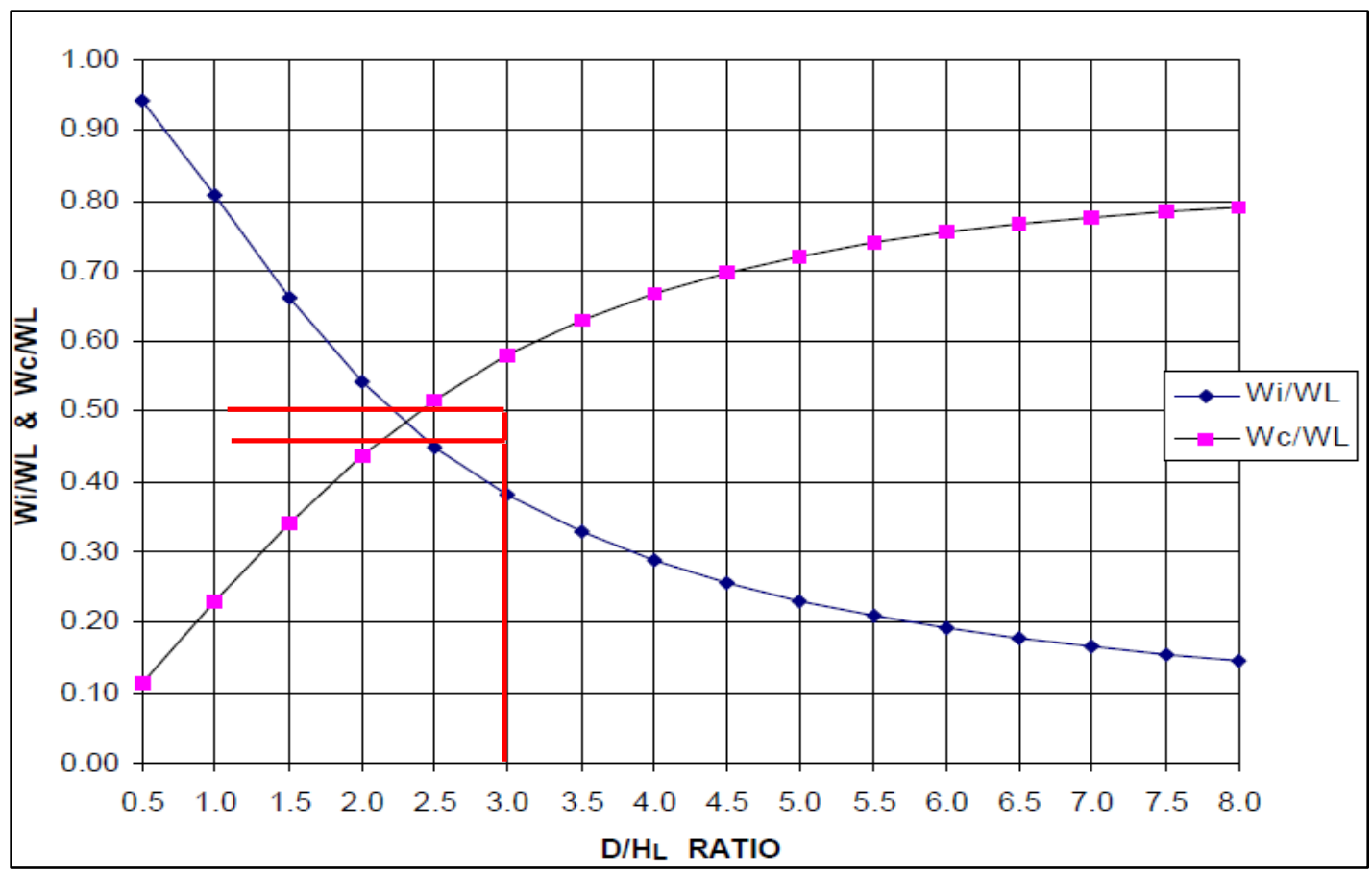

Fuente: "Seismic Design of Liquid Containing Concrete Structures" ACI 350.3. p43.

$$
\begin{aligned}
& \mathrm{He}=7.70 \mathrm{~m} \\
& \mathrm{~W}_{\mathrm{L}}=2043389.40 \mathrm{Kgf} \\
& \mathrm{W}_{\mathrm{W}}=3140188.00 \mathrm{Kgf} \\
& \mathrm{D} / \mathrm{H}_{\mathrm{L}}=2.40 \mathrm{~m} \\
& \mathrm{~W}_{\mathrm{i}} / \mathrm{W}_{\mathrm{L}}=0.50 \mathrm{~m} \\
& \mathrm{~W}_{\mathrm{C}} / \mathrm{W}_{\mathrm{L}}=0.60 \mathrm{~m} \\
& \mathrm{~W}_{\mathrm{i}}=954787.50 \mathrm{Kgf} \\
& \mathrm{W}_{\mathrm{c}}=1125307.40 \mathrm{Kgf}
\end{aligned}
$$

Cálculo de las alturas efectivas impulsivas y convectivas. ${ }^{21}$

${ }^{21}$ ACI 350.R3-1 (2008): 45 
Figura 15-II: Verificación de he/HLy hi/HL por gráfico.

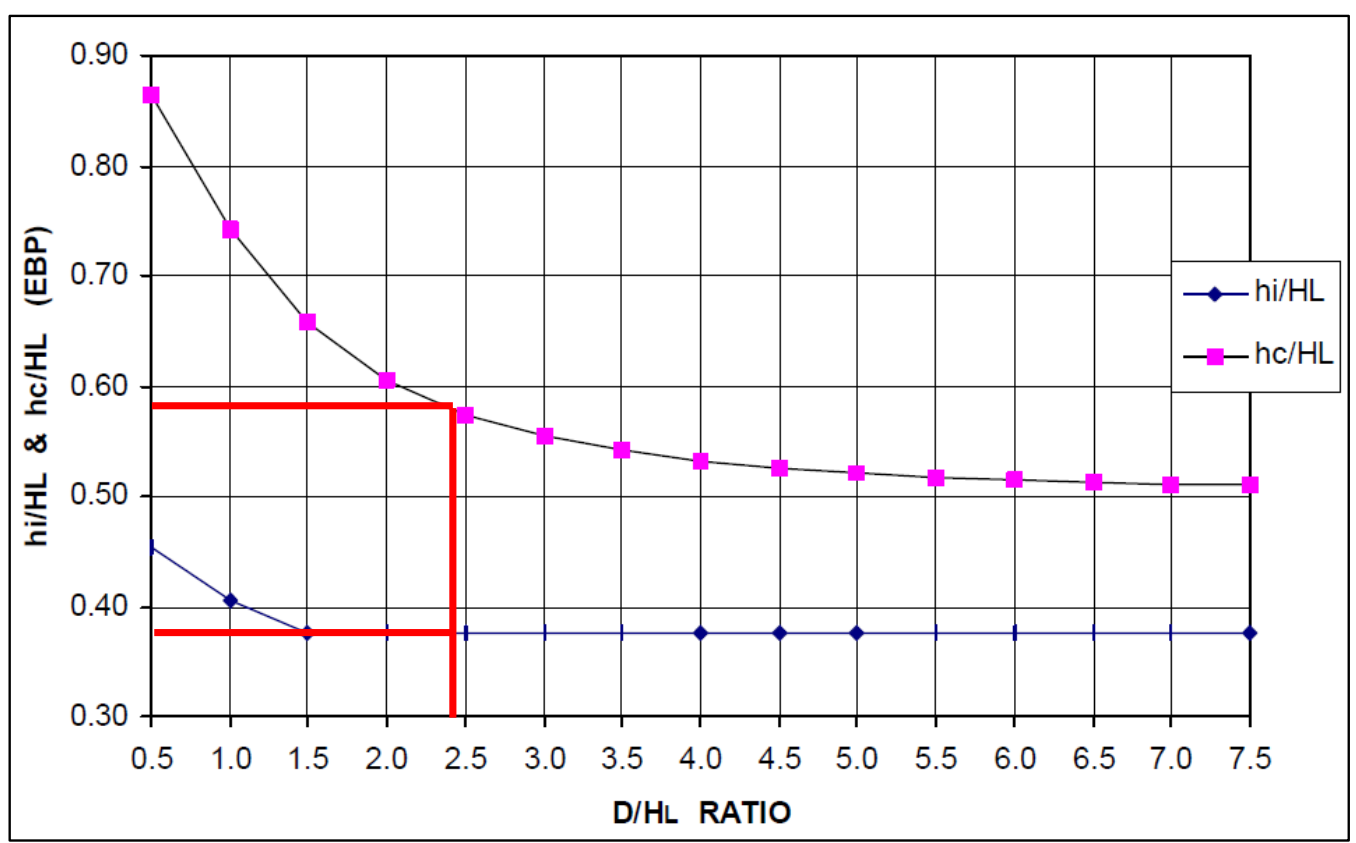

Fuente: "Seismic Design of Liquid Containing Concrete Structures” ACI 350.3. p44.

$\mathrm{D} / \mathrm{H}_{\mathrm{L}}=2.40 \mathrm{~m}$

$\mathrm{h}_{\mathrm{i}} / \mathrm{HL}_{\mathrm{L}}=0.89$

$\mathrm{h}_{\mathrm{C}} / \mathrm{H}_{\mathrm{L}}=0.38$

$\mathrm{h}_{\mathrm{C}}=6.81 \mathrm{~m}$

$\mathrm{h}_{\mathrm{i}}=2.88 \mathrm{~m}$

\subsection{METRADO DE CARGAS}

Para poder obtener el metrado de cargas del tanque elevado, se tiene que realizar el metrado de cada estructura que la compone, las cuales se han mencionado anteriormente. A continuación, se muestran los cálculos para hallar el peso total de la estructura.

Cúpulas

Cúpula Superior

En la figura 15-II se muestra las dimensiones de la cúpula superior. 
Figura 16-II: Vistas de la Cúpula Superior.
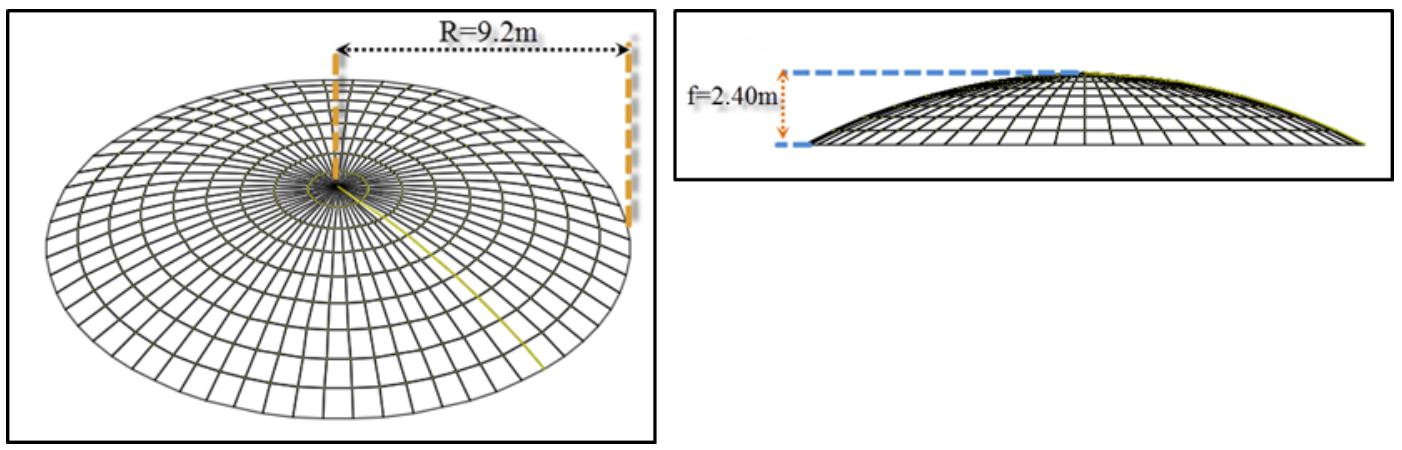

Fuente: Elaboración Propia.

$$
A=\pi\left(r^{2}+f^{2}\right)
$$

$$
V=\text { AreaxEspesor }
$$

$$
\begin{aligned}
& \text { Área }=284.00 \mathrm{~m} 2 \\
& \mathrm{r}=9.2 \mathrm{~m} \\
& \mathrm{f}=\quad 2.4 \mathrm{~m} \\
& \mathrm{e}=\quad 0.075 \mathrm{~m}
\end{aligned}
$$$$
\mathrm{V}=21.30 \mathrm{~m} 3
$$

$$
\begin{aligned}
& \text { Peso }=\text { Volumen } \times 2.4 \mathrm{Tn} / \mathrm{m} 3 \\
& \text { Peso }=101.39 \mathrm{Tn}
\end{aligned}
$$

Donde:

r: Radio de la cúpula.

f: Altura del casquete esférico.

e: Espesor de la cúpula.

\section{Cúpula Inferior}

En las figuras 16-II y 17-II, se muestran las dimensiones y vistas de la cúpula inferior.

Vista de perfil: 
Figura 17-II: Cúpula Inferior (Vista perfil).

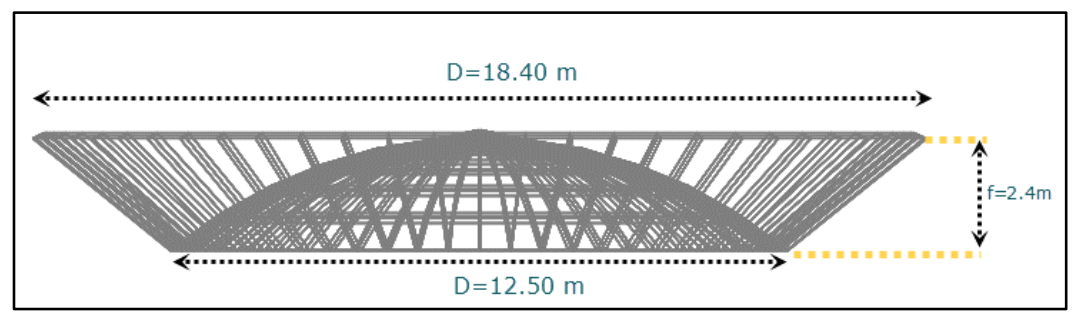

Fuente: Elaboración Propia.

Figura 18-II: Cúpula Inferior (Vista 1).

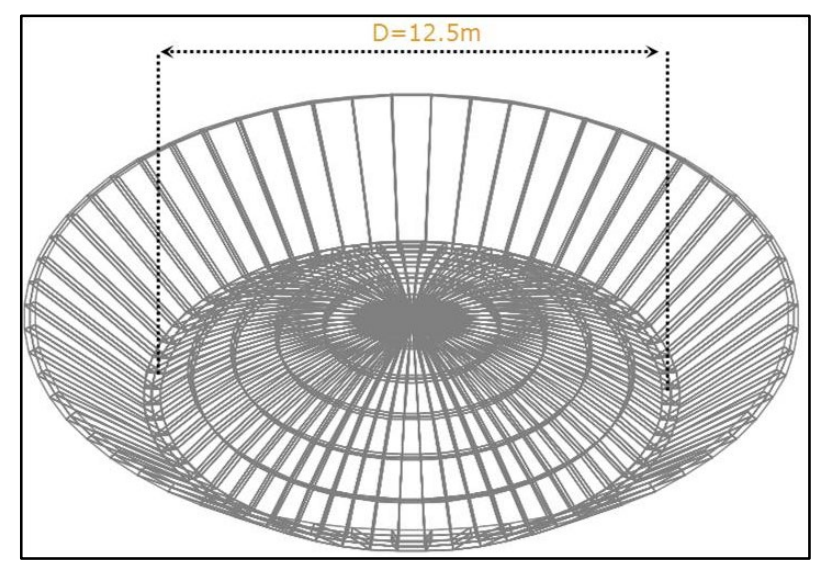

Fuente: Elaboración Propia.

$$
\begin{aligned}
& A=\pi\left(r^{2}+f^{2}\right) \\
& V=\text { AreaxEspesor } \\
& \text { Área }=140.81 \mathrm{~m} 2 \\
& \mathrm{~V}=42.24 \mathrm{~m} 3 \\
& \mathrm{r}=6.25 \mathrm{~m} \\
& \mathrm{f}=2.4 \quad \mathrm{~m} \\
& \mathrm{e}=0.3 \mathrm{~m} \\
& \text { Peso }=\text { Volumen } \times 2.4 \mathrm{Tn} / \mathrm{m} 3 \\
& \text { Peso }=101.39 \mathrm{Tn}
\end{aligned}
$$

Donde:

r: Radio de la cúpula.

f: Altura del casquete esférico.

e: Espesor de la cúpula.

\section{Vigas}


En las figuras siguientes (Figura 18-II ,19-II y 20-II) se muestran las medidas respectivas para las vigas que conforman la estructura en diferentes vistas.

\section{Viga Superior}

Figura 19-II: Vista de la Viga Superior.

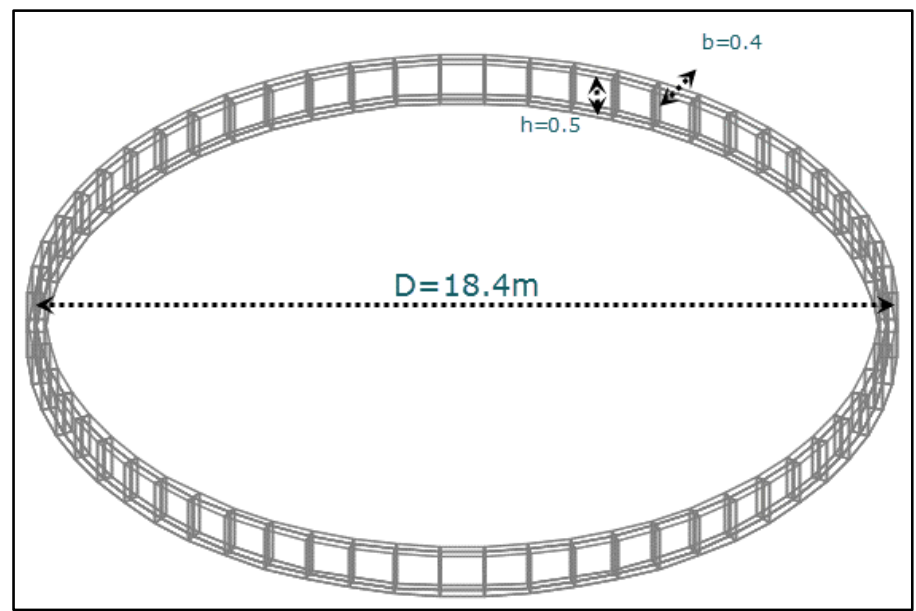

Fuente: Elaboración Propia.

$$
\begin{aligned}
& P=2 \pi \cdot r \\
& V=P . b . h \\
& \mathrm{P}=57.81 \mathrm{~m} \\
& \mathrm{~V}=11.56 \mathrm{~m} 3 \\
& \mathrm{r}=9.2 \mathrm{~m} \\
& \mathrm{~b}=0.4 \mathrm{~m} \\
& \mathrm{~h}=0.5 \mathrm{~m} \\
& \text { Peso }=\text { Volumen } \times 2.4 \mathrm{Tn} / \mathrm{m} 3 \\
& \text { Peso }=27.75 \text { Tn }
\end{aligned}
$$

Donde:

P: Perímetro.

r: Radio de la viga.

V: Volumen de la viga.

b: Espesor de la viga.

h: Altura de la viga. 
Viga Inferior

Figura 20-II: Vista de la Viga Inferior.

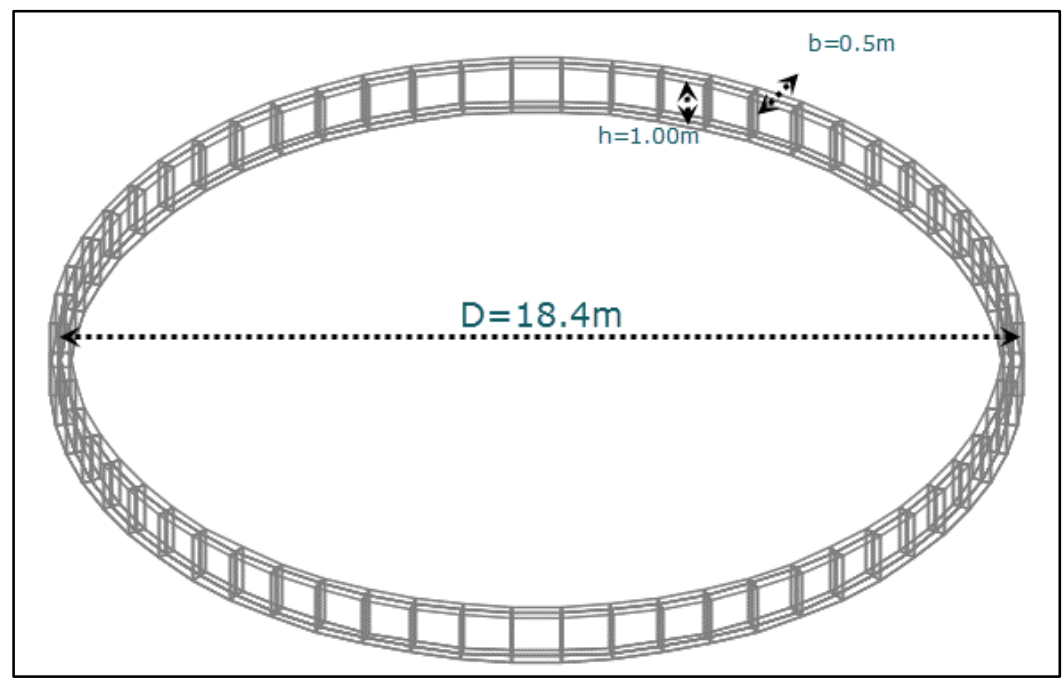

Fuente: Elaboración Propia.

\begin{tabular}{|c|c|c|c|c|}
\hline \multicolumn{2}{|c|}{$P=2 \pi \cdot r$} & & \multicolumn{2}{|c|}{$V=P . b . h$} \\
\hline $\mathrm{P}=$ & $57.81 \mathrm{~m}$ & & $\mathrm{~V}=$ & $28.90 \mathrm{~m} 3$ \\
\hline \multirow[t]{2}{*}{$\mathrm{r}=$} & 9.2 & & $b=$ & 0.5 \\
\hline & & $\mathrm{h}=$ & 1.0 & $\mathrm{mx}$ \\
\hline Peso & Volumen $x$ & & & \\
\hline
\end{tabular}

Donde:

P: Perímetro.

r: Radio de la viga.

V: Volumen de la viga.

b: Espesor de la viga.

h: Altura de la viga. 
Viga del Fuste

Figura 21-II: Vista de la Viga del Fuste.

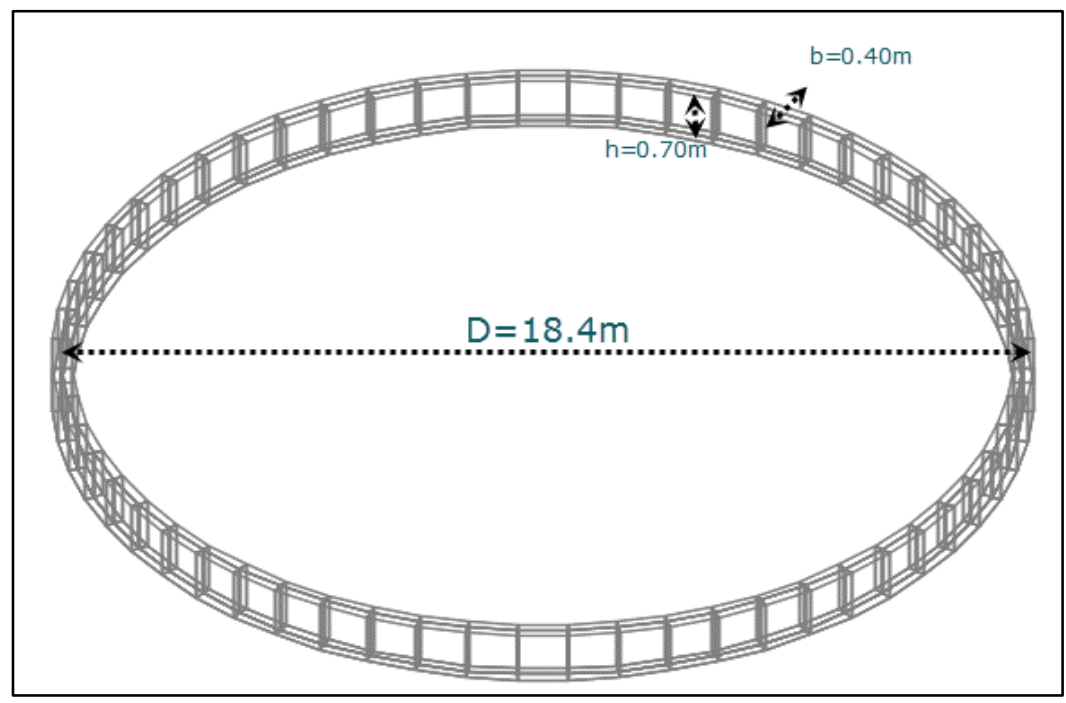

Fuente: Elaboración Propia. .

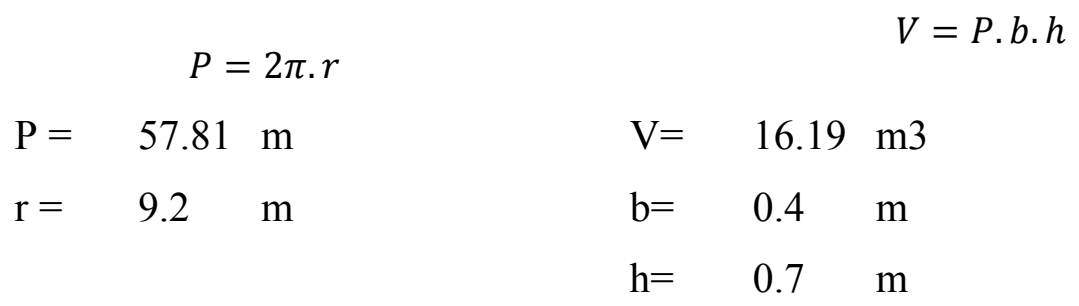

Peso $=$ Volumen $\times 2.4 \mathrm{Tn} / \mathrm{m} 3$

Peso $=38.85$ Tn

Donde:

P: Perímetro.

r: Radio de la viga.

$\mathrm{V}$ : Volumen de la viga.

b: Espesor de la viga.

h: Altura de la viga. 
Fondo Troncocónico

En la figura 21-II se muestra las medidas del fondo troncocónico.

Figura 22-II: Vista del Fondo Troncocónico.

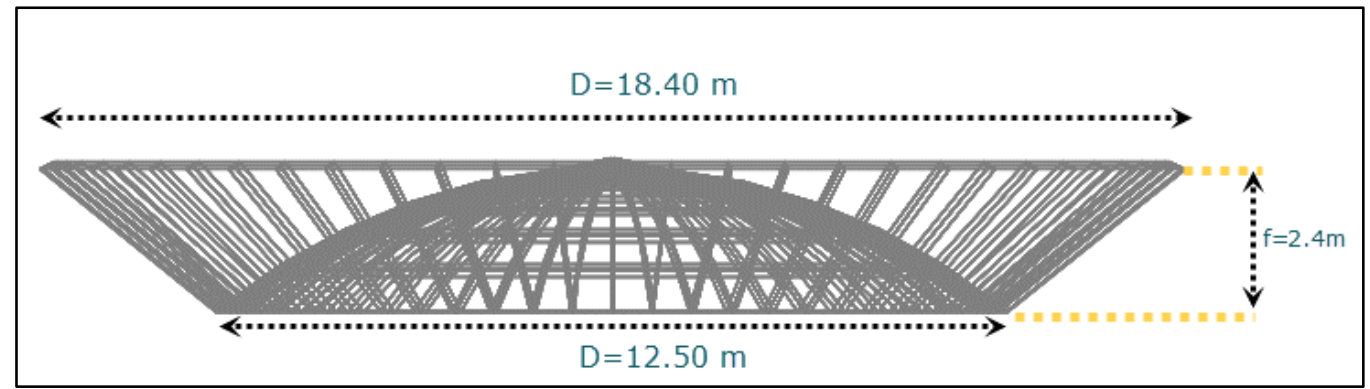

Fuente: Elaboración Propia.

Area Superficie Cónica $=\pi \cdot(R+r) \cdot g$

$$
\begin{array}{lllll}
\mathrm{h}= & 2.4 & \mathrm{~m} & & \\
\mathrm{R}= & 9.2 & \mathrm{~m} & \mathrm{~A}= & 184.59 \mathrm{~m} 2 \\
\mathrm{r}= & 6.25 & \mathrm{~m} & \mathrm{~V}=64.61 \mathrm{~m} 3 \\
\mathrm{~g}= & 3.80 & \mathrm{~m} & & \\
\mathrm{e}= & 0.35 & \mathrm{~m} & &
\end{array}
$$$$
\text { Peso }=\text { Volumen } \times 2.4 \mathrm{Tn} / \mathrm{m} 3
$$$$
\text { Peso }=155.05 \mathrm{Tn}
$$

Donde:

$\mathrm{g}:$ Generatriz.

h: Altura del cono truncado.

$\mathrm{R}$ : Radio mayor del cono.

$\mathrm{r}$ : Radio menor del cono.

e : Espesor la superficie cónica. 
Fuste

En la figura 22-II se muestran las medidas a considerar para el metrado del fuste.

Figura 23-II: Vista del Fuste.

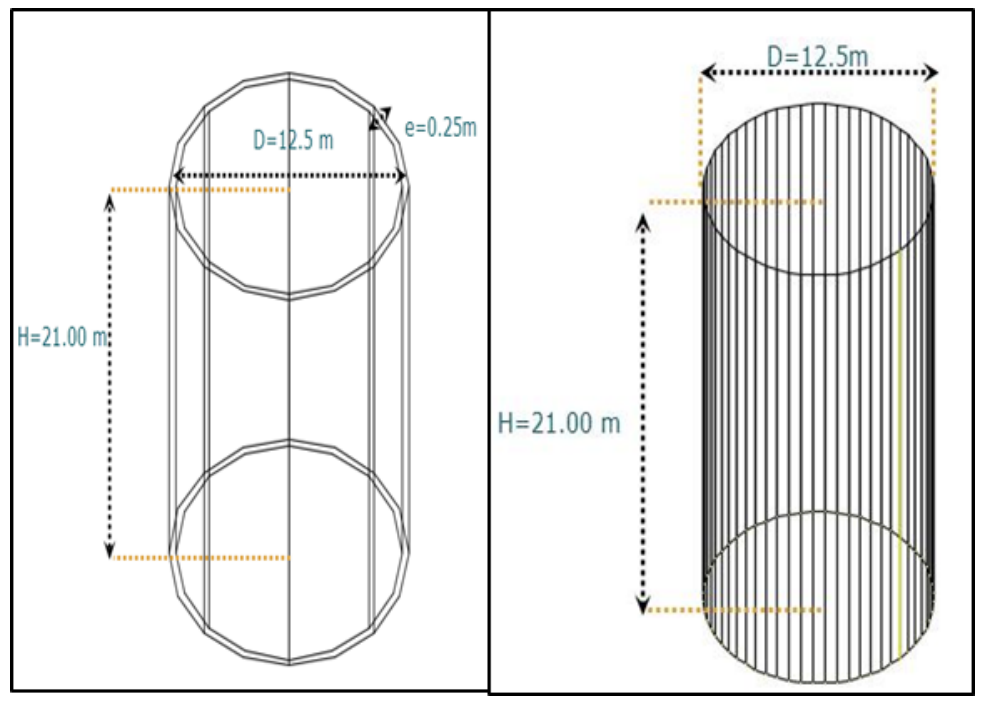

Fuente: Elaboración Propia.

$$
\begin{aligned}
& \text { Area Superficie Cilíndrica }=2 . \pi \cdot r \cdot h \quad \text { Peso }=\text { Volumen } \times 2.4 \mathrm{Tn} / \mathrm{m} 3 \\
& \mathrm{~A}=\quad 824.67 \mathrm{~m} 2 \\
& \mathrm{r}=\quad 6.25 \mathrm{~m} \\
& \mathrm{~V}=\quad 206.17 \mathrm{~m} 3 \\
& \mathrm{~h}=21 \mathrm{~m} \\
& \text { Peso }=593.76 \mathrm{Tn} \\
& \mathrm{e}=\quad 0.30 \mathrm{~m}
\end{aligned}
$$

Donde:

$\mathrm{r}$ : Radio del fuste.

h: Altura del fuste.

e : Espesor del fuste.

Chimenea de Acceso 
En la figura 23-II se muestran las medidas a considerar para el metrado de la chimenea de acceso.

Figura 24-II: Vista de la Chimenea de Acceso.

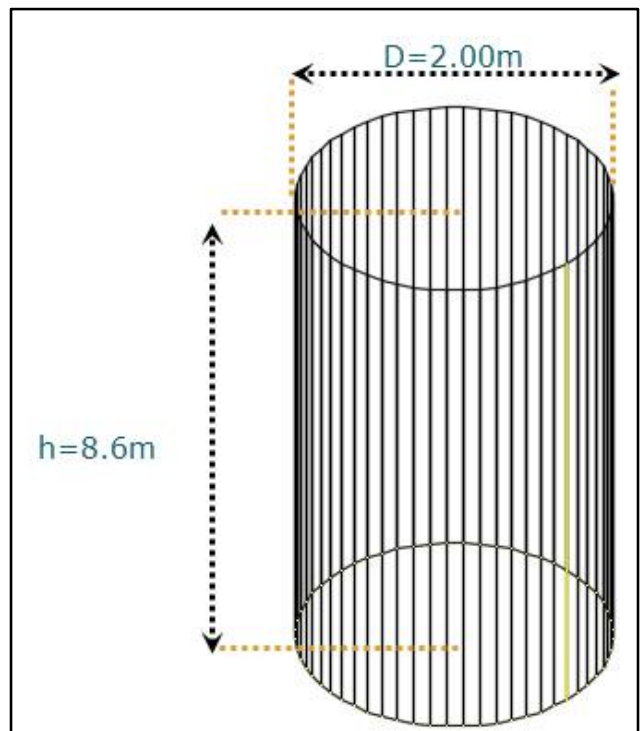

Fuente: Elaboración Propia.

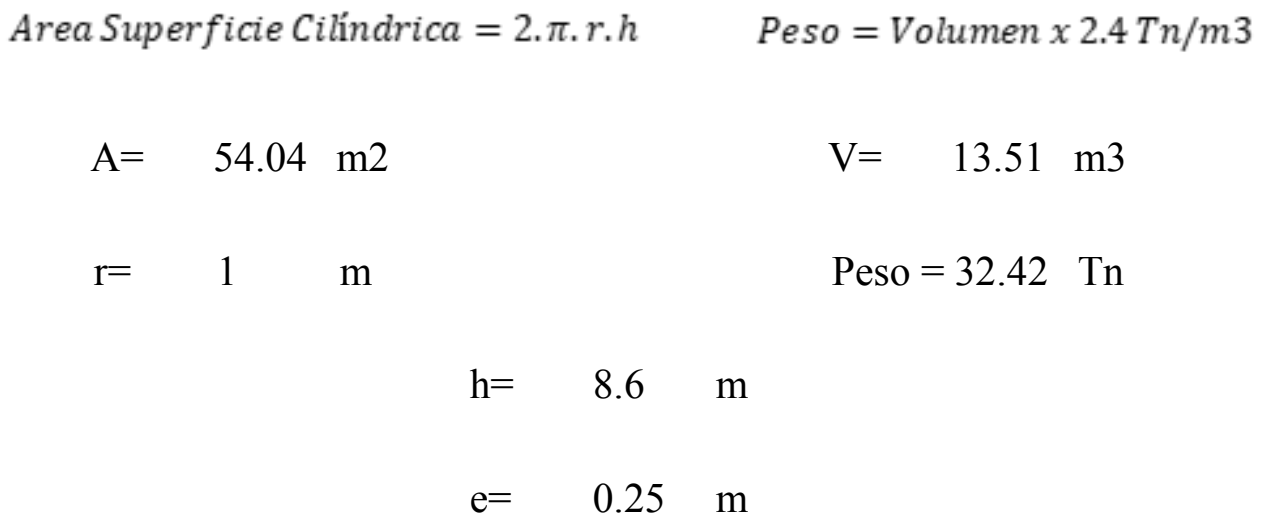

Donde:

r : Radio de la chimenea de acceso.

h: Altura de la chimenea de acceso.

e : Espesor de la chimenea de acceso.

Cuba 
En la figura 24-II, se muestra las medidas a considerar para el metrado de carga muerta del elemento estructural cuba.

Figura 25-II: Vista de la Cuba.

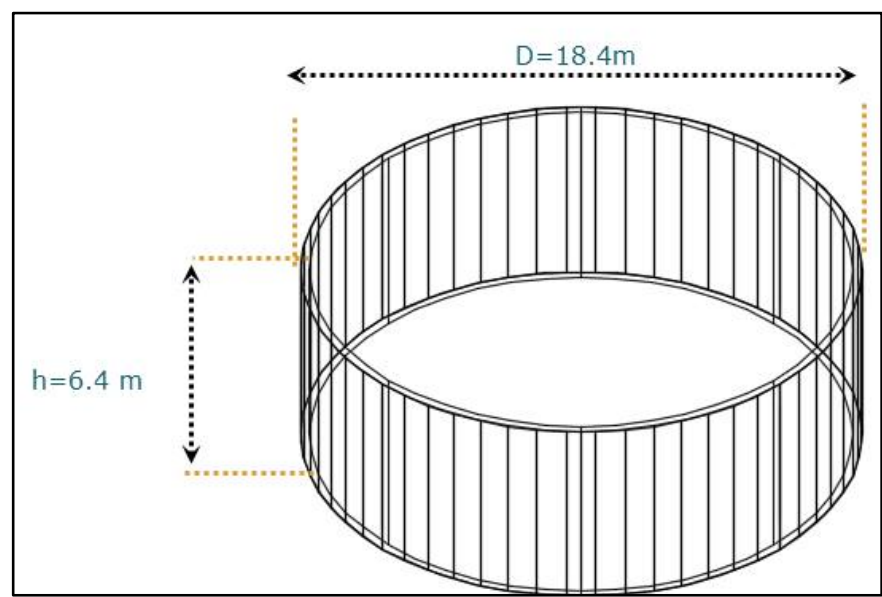

Fuente: Elaboración Propia.

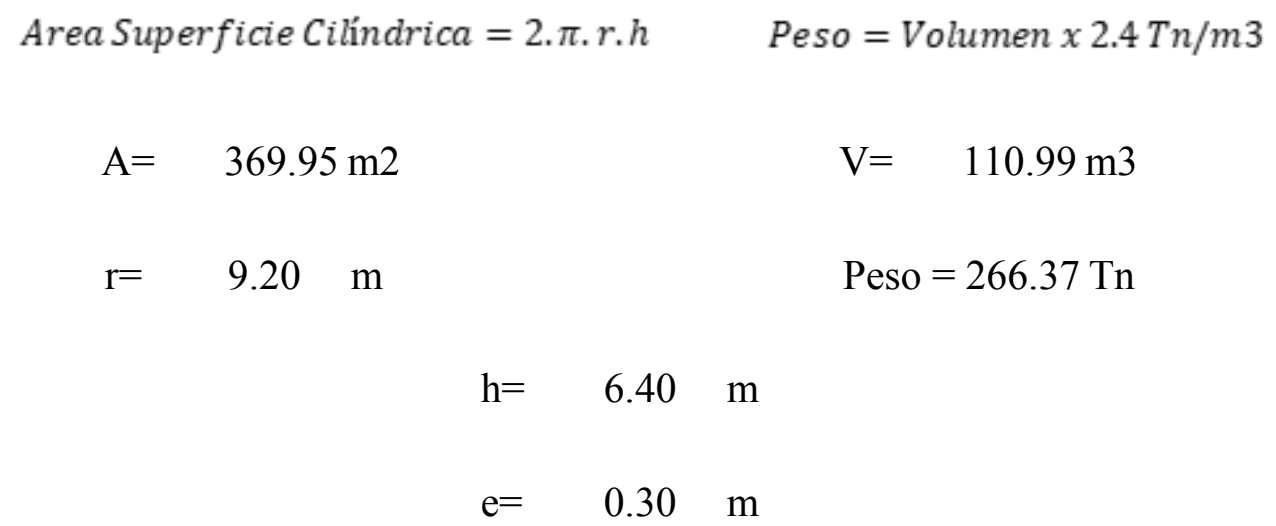

Donde:

$\mathrm{r}$ : Radio de la cuba.

h: Altura de la cuba.

e : Espesor de la cuba.

Resumen de Metrado de Elementos Estructurales 


\section{Cúpulas}

Cúpula Superior $(\mathrm{e}=0.075 \mathrm{~m})$

Cúpula Inferior $(\mathrm{e}=0.30 \mathrm{~m})$

Metrado de Vigas

Viga Superior

Viga Inferior

Viga del Fuste

Fondo tronco-cónico

Fuste

Chimenea de Acceso

Cuba

Peso Estructura

Peso Total
PESO

\begin{tabular}{|r|}
\hline $51.12 \mathrm{Tn}$ \\
\hline $101.39 \mathrm{Tn}$ \\
\hline
\end{tabular}

\begin{tabular}{|rl|}
\hline 27.75 & $\mathrm{Tn}$ \\
\hline 69.37 & $\mathrm{Tn}$ \\
\hline 38.85 & $\mathrm{Tn}$ \\
\hline 155.05 & $\mathrm{Tn}$ \\
\hline 593.76 & $\mathrm{Tn}$ \\
\hline 32.42 & $\mathrm{Tn}$ \\
\hline 266.37 & $\mathrm{Tn}$ \\
\hline
\end{tabular}

$1323.61 \mathrm{Tn}$

$3323.61 \mathrm{Tn}$ 


\section{CAPÍTULO III: METODOLOGÍA}

\subsection{ANÁLISIS ESTRUCTURAL}

De acuerdo a los cálculos realizados, las cargas que actúan en la estructura, las masa impulsiva y convectiva que actúan sobre la cuba y las alturas en las cuales se ubican cada una de estas masas, se procederá a un modelamiento. Con el fin de estudiar y analizar los resultados que posteriormente usamos para el diseño final del tanque elevado.

La evaluación de los efectos de fuerzas que actúan sobre la estructura se conforma por tres categorías: permanentes, variables y accidentales.

- Acciones permanentes: son aquellas fuerzas permanentes como las cargas muertas, que tienen un peso que no varía en el tiempo.

- Acciones variables: son aquellas cargas que varían respecto al tiempo y afectan considerablemente a la estructura. Las acciones variables son: la carga viva, el empuje estático del líquido y los efectos de cambio de temperatura ${ }^{22}$.

- Acciones accidentales: son aquellas cargas o acciones que se debe a fuerzas externas que se generan por lapsos amplios o mínimos, las cuales generan, de acuerdo a su intensidad, daños considerables a la estructura. Estas acciones son; fuerzas sísmicas, explosiones, incendios y otros fenómenos que se podría $\operatorname{suscitar}^{23}$.

Para el caso de la presión de agua, la cuba está sometida a fuerzas hidrostáticas. Así mismo, el diagrama de esfuerzos se aplicará en los distintos puntos de estudio que se propuso según lo modelado en el SAP2000, además se considera que para el diseño final, el acero de refuerzo debe absorber estas fuerzas la cual se comporta como se muestra en la figura 25-III.

\footnotetext{
${ }^{22}$ Cfr: Nieto, C. \& Zhañay, W.2011: 20

${ }^{23}$ Cfr: Nieto, C. \& Zhañay, W.2011: 20
} 
Figura 26-III: Cargas hidráulicas en la cuba

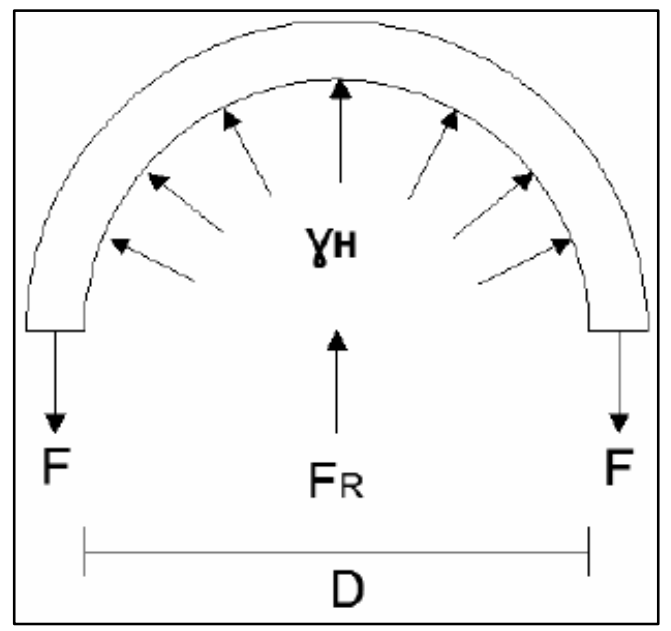

Fuente: "Diseño de un tanque apoyado de ferrocemento para la comunidad de Santa Rosa de Chichin, perteneciende a la parroquia Jadán del Cantón Gualaceo”. C.Nieto y W. Zhañay. p20.

Para la generación del modelo estructural, proponemos los cálculos y medidas tentativas que se calculó previamente.

\subsection{ANÁLISIS SÍSMICO ESTÁTICO}

De acuerdo los establecido en el Reglamento Nacional de Edificaciones E 030, se elegirán unos factores, dado los efectos de Zona Z, Uso o importancia U, Suelo S y su periodo del terreno asociado Tp, así como el coeficiente de amplificación sísmica $\mathrm{C}$, son los mismos para todo el sistema de análisis, además, como esta norma no contempla el factor R (factor de modificación de respuesta), se usará el R que nos brinda el ACI.

Zonificación (Z): Este factor representa la aceleración máxima del terreno, para el presente proyecto nuestra edificación estará ubicada en la ciudad de Lima la cual corresponde a la zona 4 con un factor $Z=0.45^{24}$.

${ }^{24}$ Cfr: NTE-E030 (2016): 1 
Figura 27-III: Zonificación Sísmica

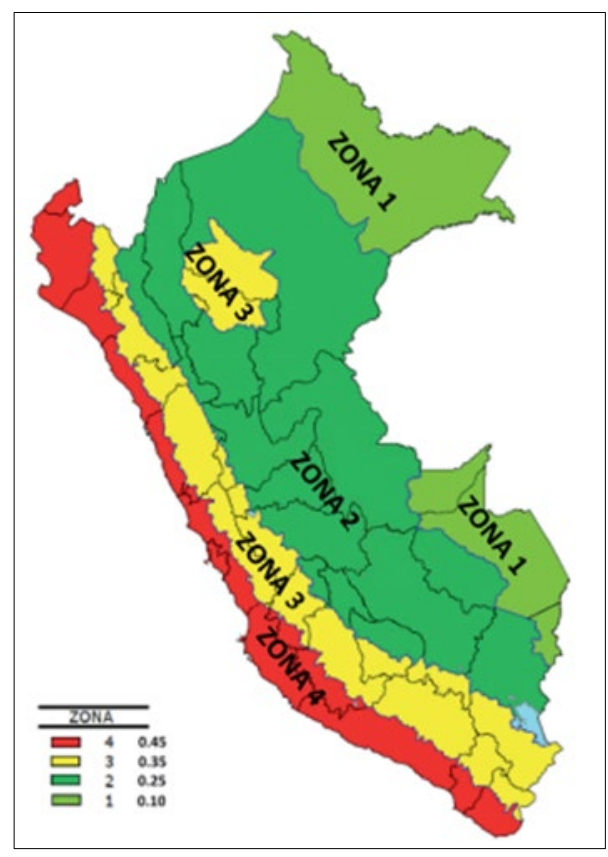

Fuente: "Diseño Sismoresistente" E030-2016. p8.

Tabla 5-III: Zonificación Sísmica

\begin{tabular}{|c|c|}
\hline \multicolumn{2}{|c|}{ FACTORES DE ZONA } \\
\hline ZONA & Z \\
\hline 4 & 0.45 \\
\hline 3 & 0.35 \\
\hline 2 & 0.25 \\
\hline 1 & 0.10 \\
\hline
\end{tabular}

Fuente: “Diseño Sismoresistente” E030-2016. p8.

Parámetro del suelo ( $\mathbf{T}$ y S): para la selección de tipo de suelo se clasifica tomando propiedades mecánicas, el espesor del estrato y la velocidad de propagación. ${ }^{25}$

${ }^{25}$ Cfr: RNE-E030 (2016): 2 
Tabla 5-III: Factor "S" suelo para determinación de parámetros sísmicos

\begin{tabular}{|c|c|c|c|c|}
\hline Suelo & $\mathrm{S}_{0}$ & $\mathrm{~S}_{1}$ & $\mathrm{~S}_{2}$ & $\mathrm{~S}_{3}$ \\
\hline $\mathrm{Z}_{4}$ & 0.80 & 1.00 & 1.05 & 1.10 \\
\hline $\mathrm{Z}_{3}$ & 0.80 & 1.00 & 1.15 & 1.20 \\
\hline $\mathrm{Z}_{2}$ & 0.80 & 1.00 & 1.20 & 1.40 \\
\hline $\mathrm{Z}_{1}$ & 0.80 & 1.00 & 1.60 & 2.00 \\
\hline
\end{tabular}

Fuente: "Diseño Sismoresistente" E030-2016. P12.

Tabla 6-III: Parámetros para la determinación de periodos fundamentales.

\begin{tabular}{|c|c|c|c|c|}
\hline \multicolumn{5}{|c|}{ Periodo "TP " $y$ "TL" } \\
\hline & $\mathrm{S}_{0}$ & $\mathrm{~S}_{1}$ & $\mathrm{~S}_{2}$ & $\mathrm{~S}_{3}$ \\
\hline $\mathrm{T}_{\mathrm{p}(\mathrm{S})}$ & 0.30 & 0.40 & 0.60 & 1.0 \\
\hline $\mathrm{T}_{\mathrm{L}(\mathrm{S})}$ & 3.00 & 2.50 & 2.0 & 1.60 \\
\hline
\end{tabular}

Fuente: “Diseño Sismoresistente” E030-2016. p12.

Factor de Amplificación sísmica (C): esta característica se basa en los parámetros del suelo mediante la siguiente formula.

$$
\begin{array}{cc}
T<T_{p} & C=2.5 \\
T_{p}<T<T_{L} \quad C=2,5 .\left(\frac{T_{p}}{T}\right) \\
T>T_{L} \quad C=2,5 .\left(\frac{T_{p} \cdot T_{L}}{T^{2}}\right)
\end{array}
$$

En las ecuaciones (25) se muestran las fórmulas a seguir para hallar el factor de amplificación sísmica de acuerdo al periodo que se debe seguir según norma E030.

Categoría de Edificación (U): Cada estructura se clasifica mediante su importancia y uso, debido a que el tanque elevado la norma establece un factor de importancia $U=1.50$, mediante la siguiente tabla. ${ }^{26}$

${ }^{26}$ Cfr: RNE-E030 (2016): 3 
Tabla 7-III: Categoría de Edificaciones

\begin{tabular}{|c|c|c|}
\hline \multicolumn{3}{|c|}{ CATEGORIA DE LAS EDIFICACIONES } \\
\hline CATEGORIA & DESCRIPCIÓN & FACTOR U \\
\hline & $\begin{array}{l}\text { A1: Establecimientos de salud, como hospitales, institutos o similares, } \\
\text { según clasificación del Ministerio de Salud, ubicados en las zonas } \\
\text { sísmicas } 4 \text { y } 3 \text { que alojen cualquiera de los servicios indicados en la } \\
\text { Tabla № } 5.1 \text {. }\end{array}$ & Ver nota 1 \\
\hline $\begin{array}{l}\text { A } \\
\text { Edificaciones } \\
\text { Esenciales }\end{array}$ & $\begin{array}{l}\text { A2: Edificaciones esenciales cuya función no debería interrumpirse } \\
\text { inmediatamente después de que ocurra un sismo severo tales como: } \\
\text { - Hospitales no comprendidos en la categoría A1, clínicas, postas } \\
\text { médicas, excepto edificios administrativos o de consulta externa. (Ver } \\
\text { nota 2) } \\
\text { - Puertos, aeropuertos, centrales de comunicaciones. Estaciones de } \\
\text { bomberos, cuarteles de las fuerzas armadas y policía. } \\
\text { - Instalaciones de generación y transformación de electricidad, } \\
\text { reservorios y plantas de tratamiento de agua. } \\
\text { Todas aquellas edificaciones que puedan servir de refugio después de } \\
\text { un desastre, tales como colegios, institutos superiores tecnológicos y } \\
\text { universidades. } \\
\text { Se incluyen edificaciones cuyo colapso puede representar un riesgo } \\
\text { adicional, tales como grandes hornos, fábricas y depósitos de materiales } \\
\text { inflamables o tóxicos. }\end{array}$ & 1.5 \\
\hline $\begin{array}{l}\text { B } \\
\text { Edificaciones } \\
\text { Importantes }\end{array}$ & $\begin{array}{l}\text { Edificios en centros educativos y de salud no incluidos en la categoría A. } \\
\text { Edificaciones donde se reúnen gran cantidad de personas tales como } \\
\text { teatros, estadios, centros comerciales, terminales de pasajeros, } \\
\text { establecimientos penitenciarios, o que guardan patrimonios valiosos } \\
\text { como museos, bibliotecas y archivos especiales. } \\
\text { También se considerarán depósitos de granos y otros almacenes } \\
\text { importantes para el abastecimiento }\end{array}$ & 1.3 \\
\hline$\frac{\text { C }}{\text { Edificaciones Comunes }}$ & $\begin{array}{l}\text { Edificaciones comunes tales como: viviendas, oficinas, hoteles, } \\
\text { restaurantes, depósitos e instalaciones industriales cuya falla no acarree } \\
\text { peligros adicionales de incendios o fugas de contaminantes. }\end{array}$ & 1.0 \\
\hline$\frac{\text { D }}{\text { Edificaciones Menores }}$ & Construcciones provisionales para depósitos, casetas y otras similares. & Ver nota 3 \\
\hline
\end{tabular}

Fuente: "Diseño Sismo resistente" E030-2018. p13.

Nota 1: Estas edificaciones tendrán aislamiento sísmico en la base, excepto en condiciones de suelo desfavorables al uso del sistema de aislamiento.

Nota 2: Estas edificaciones tendrán un sistema de protección sísmica por aislamiento o disipación de energía cuando se ubiquen en las zonas sísmicas 4 y 3.

Nota 3: En estas edificaciones deberá proveerse resistencia y rigidez adecuadas para acciones laterales, a criterio del proyectista. 
La estructura presenta aberturas en el fuste; sin embargo, se adopta un modelo estructural regular.

Factor de Reducción (R): Para este caso se usarán dos factores para las dos distintas masas que presenta la estructura basándonos en el ACI.

Tabla 8-III: Tabla de Coeficientes de Reducción.

\begin{tabular}{|l|c|c|c|}
\hline \multicolumn{1}{|c|}{ Tipo de estructura } & $\begin{array}{c}\mathbf{R} \\
\text { impulsiva }\end{array}$ & $\begin{array}{c}\mathbf{R} \\
\text { Enterrado }\end{array}$ & $\begin{array}{c}\mathbf{R} \\
\text { convectiva }\end{array}$ \\
\hline (a)Tanques anclados con base flexible & 3.25 & 3.25 & 1.0 \\
\hline $\begin{array}{l}\text { (b) Tanques monolíticos o empotrado en la } \\
\text { base }\end{array}$ & 2.0 & 3.0 & 1.0 \\
\hline (c) Tanques no anclados & 1.5 & 2.0 & 1.0 \\
\hline (d) Tanques elevados & $\mathbf{2}$ & - & 1.0 \\
\hline
\end{tabular}

Fuente: "Seismic Design of Liquid Containing Concrete Structures" ACI 350.3. p21.

Para el análisis estático se tiene que tomar consideraciones del ACI para estructuras contenedoras de líquido. Como se calculó previamente, para el factor de reducción de $\mathbf{R}=\mathbf{2}$ para las direcciones $\mathrm{X}$ e $\mathrm{Y}$ basándonos en la norma para la masa impulsiva y $\mathbf{R}=\mathbf{1}$ para la convectiva ACI $350.3 .^{27}$

Teniendo como base este factor de reducción calcularemos la cortante en la base debido a un sismo como lo contempla la norma E030-2016.

$$
\begin{aligned}
\mathrm{Z} & =0.45 \\
\mathrm{U} & =1.5 \\
\mathrm{~S} & =1 \\
\mathrm{C} & =1.74 \\
\mathrm{Ri}= & 2 ; \mathrm{Rc}=1
\end{aligned}
$$

${ }^{27}$ Cfr: ACI 350.3 (2008): 20 
Factor de Amplificación Sísmica

Este factor depende del período "T" de acuerdo al numeral 4.5.4. De la norma E.030 como se muestra en la Ecuación 26.

$$
T=\frac{h_{n}}{C_{T}}
$$

Donde:

$\mathrm{hn} \quad=\quad 34.4 \mathrm{~m}$; Altura total de la estructura.

$\mathrm{CT}=60 \quad$; coeficiente para estimar el periodo predominante de un edificio.

$\mathrm{T}=0.57$; análisis estático o periodo de un modo en el análisis dinámico.

Del numeral 2.4. De la norma E.030

$\mathrm{TL}=2.5 \mathrm{~s}$

$\mathrm{TP}=0.4 \mathrm{~s}$

De la norma ACI 350.3 ecuación (9-25), se obtiene el periodo del tanque con las consideraciones de la masa impulsiva. La cual se calcula como lo muestra la Ecuación 27.

$$
T_{i}=\frac{2 \pi}{w_{i}}
$$

Donde $\mathrm{w}_{\mathrm{i}}$ es el peso equivalente del componente impulsivo del líquido almacenado (Ecuación 9 - 23 del ACI 350.3)

$\mathrm{H}_{\mathrm{L}}=$ Altura total del líquido

$$
w_{i}=C_{I} \cdot \frac{12}{H_{L}} \cdot \sqrt{E_{c} \cdot \frac{g}{\gamma_{C}}}
$$

$\mathrm{E}_{\mathrm{C}}=$ Módulo de elasticidad del concreto Tn/m2 $2028792.74($ Para f'c=210 kg/cm2) $\quad \gamma \mathrm{c}$ =Peso específico del concreto Tn/m3

A su vez, wi depende de: 


$$
w_{i}=C_{I} \cdot \frac{12}{H_{L}} \cdot \sqrt{E_{c} \cdot \frac{g}{\gamma_{C}}}
$$

$\mathrm{C}_{\mathrm{I}}, \mathrm{C}_{\mathrm{w}}=$ Coeficiente para determinar la frecuencia fundamental del sistema (tanque - líquido). Ecuación 9 - 24 del ACI 350.3-06

$\mathrm{C}_{\mathrm{w}}=$ De la fig. 9.3.4(a) del ACI 350.3 - 06

$\mathrm{t}_{\mathrm{w}}=$ Espesor de la pared

$\mathrm{r} \quad=$ Radio del tanque circular

Coeficiente $\mathrm{Cw}$

Figura 28-III: Coeficiente $\mathrm{CW}$

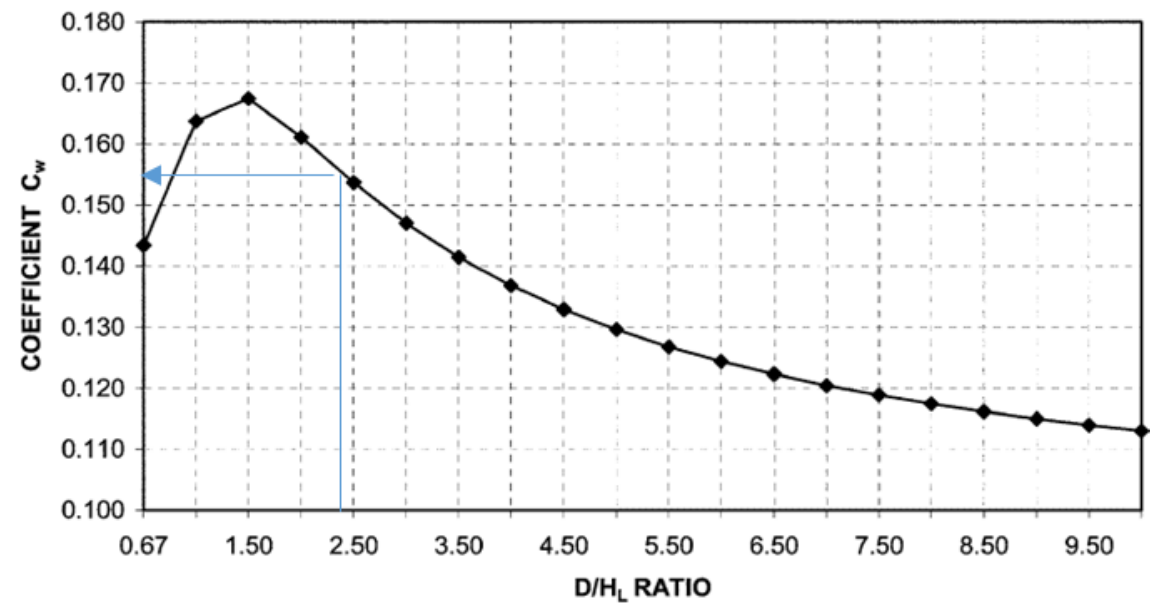

Fuente: "Seismic Design of Liquid Containing Concrete Structures" ACI 350.3. p50.

\begin{tabular}{|l|l|}
\hline $\mathrm{C}_{\mathrm{W}}=$ & 0.155 \\
\hline $\mathrm{C}_{\mathrm{I}}=$ & 0.0063 \\
\hline
\end{tabular}

Reemplazando en las ecuaciones anteriores:

$\begin{array}{lll}\mathrm{W}_{\mathrm{i}} & = & 28.76 \\ \mathrm{~T}_{\mathrm{i}} & = & 0.2185\end{array}$


La norma ACI 350 - 06 también da una Ecuación 30 para calcular el periodo natural del primer modo convectivo.

$$
T_{C}=\frac{2 \pi}{\lambda} \sqrt{D}
$$

El factor $2 \pi / \lambda$ se obtiene de la siguiente Ecuación 31 del ACI 350 - 06

\begin{tabular}{|l|l|l|l|}
\hline \multicolumn{2}{|c|}{$\lambda=\sqrt{3.68 \cdot g \cdot \tanh \left(3.68\left(\frac{H_{L}}{D}\right)\right.}$} & (31) \\
\hline$\lambda=$ & 5.7186 & \\
\cline { 1 - 1 }$=$ & 4.7130 & \\
\cline { 1 - 2 } & & \\
\cline { 1 - 2 } & &
\end{tabular}

Para los coeficientes de amplificación sísmica, según la norma ACI se tiene lo siguiente:

$\mathrm{Cc}=$ Factor de amplificación sísmica de la componente convectiva la cual se muestra en la Ecuación 32 (ACI 350.06)

Para:

$\mathrm{Tc} \geq 2.4 \mathrm{~s}$

$$
C c=\frac{6.0}{T_{C}^{2}}
$$

$\begin{array}{ll}\mathrm{Cc}= & 0.2701 \\ & \\ \end{array}$

Ci=Factor de amplificación sísmica de la componente impulsiva Ecuación 33 (ACI 350.06)

Para:

$$
\mathrm{T}_{\mathrm{i}} \leq 0.31 \mathrm{~s}
$$

$$
\mathrm{Ci}=2.7500
$$

La Cortante Basal se calcula mediante la siguiente Ecuación 34 de la norma ACI 350-1 


$$
V=\sqrt{(P i+P w+P r)^{2}+P c^{2}}
$$

Donde:

$\mathrm{P}_{\mathrm{i}}=$ Total de la fuerza impulsiva asociada a la masa impulsiva que se muestra en la siguiente Ecuación 35 (ACI 350-1)

$$
P_{i}=\operatorname{Z.S} . U . C_{i} \cdot\left(\frac{W_{i}}{R_{i}}\right)
$$

$$
\begin{array}{lll}
\mathrm{Wi}= & 917.16 & \mathrm{Tn} \\
\mathrm{Ri}= & 2.00 & \\
\mathrm{Pi}= & 851.24 & \mathrm{Tnf}
\end{array}
$$

$\mathrm{P}_{\mathrm{w}}=$ Fuerza de inercia lateral de la aceleración del muro Ecuación 36 (ACI 350- 1)

$\mathrm{Ww}=1272.49 \mathrm{Tn}$

$$
\begin{array}{ll} 
& P_{w}=\text { Z.S.U.C. } \cdot\left(\frac{\varepsilon \cdot W_{W}}{R_{i}}\right) \\
\varepsilon= & \text { Coeficiente de masa efectiva } \\
\varepsilon= & 0.64 \\
\mathrm{Ri}= & 2.00 \\
\mathrm{Pw}= & 760.59 \mathrm{Tnf}
\end{array}
$$

$\mathrm{P}_{\mathrm{r}}=$ Fuerza de inercia lateral de la aceleración de la cúpula la cual se muestra en la Ecuación 37 (ACI 350-1)

$$
\mathrm{Wr}=64.42 \mathrm{Tn}
$$

$$
P_{r}=Z . S . U . C_{i} \cdot\left(\frac{W_{r}}{R_{i}}\right)
$$




\begin{tabular}{|lll|}
\hline $\operatorname{Pr}=$ & 38.50 & Tnf \\
& & \\
\hline
\end{tabular}

$\mathrm{P}_{\mathrm{c}}=$ Total de la fuerza impulsiva asociada a la masa convectiva como se muestra en la Ecuación 38 (ACI 350-1)

$$
\mathrm{W}_{\mathrm{c}}=1019.44 \mathrm{Tn}
$$

$$
P_{c}=Z . S . U \cdot C_{i} \cdot\left(\frac{W_{c}}{R_{c}}\right)
$$

$$
\mathrm{R}_{\mathrm{c}} \quad=1.00
$$

$\mathrm{P}_{\mathrm{c}}=185.88 \quad \operatorname{Tnf}$

Entonces, la cortante basal (V) es igual a:

\begin{tabular}{lll}
\hline $\mathrm{V}=$ & 1660.77 & $\mathrm{Tn}$ \\
& & \\
\hline
\end{tabular}

Cálculo de las Fuerzas horizontales distribuidas secuencialmente

Tabla 9-III: Distribución de Fuerzas que actúan Sobre el Sistema Estructural.

\begin{tabular}{|c|r|r|r|r|r|}
\hline NIVEL & \multicolumn{1}{c|}{ Pi(tn) } & \multicolumn{1}{c|}{ hi(m) } & \multicolumn{1}{c|}{ Pi.hi } & \multicolumn{1}{c|}{ Fi } & W=Fi/L (Tn/m) \\
\hline VIGA SUP+CUP.SUP & 78.867 & 31.500 & 2484.296 & 50.299 & 0.870 \\
\hline CONVECTIVA & 1019.441 & 29.440 & 30012.345 & 607.649 & 10.512 \\
\hline IMPULSIVO & 917.163 & 27.920 & 25607.180 & 518.460 & 8.969 \\
\hline CUB+VIG+CHIM & 368.154 & 27.920 & 10278.872 & 208.113 & 3.600 \\
\hline CUP.INF+TRONC+VIG & 282.828 & 24.100 & 6816.159 & 138.004 & 2.387 \\
\hline FUSTE 7 & 84.823 & 20.500 & 1738.872 & 35.206 & 0.897 \\
\hline FUSTE 6 & 84.823 & 17.500 & 1484.403 & 30.054 & 0.765 \\
\hline FUSTE 5 & 84.823 & 14.500 & 1229.934 & 24.902 & 0.634 \\
\hline FUSTE 4 & 84.823 & 11.500 & 975.465 & 19.750 & 0.503 \\
\hline FUSTE 3 & 84.823 & 8.500 & 720.996 & 14.598 & 0.372 \\
\hline FUSTE 2 & 84.823 & 5.500 & 466.527 & 9.446 & 0.241 \\
\hline FUSTE 1 & 84.823 & 2.500 & 212.058 & 4.293 & 0.109 \\
\hline TOTAL & $\mathbf{3 2 6 0 . 2 1 4}$ & - & $\mathbf{8 2 0 2 7 . 1 0 4}$ & $\mathbf{1 6 6 0 . 7 7}$ & $\mathbf{4 2 . 2 9 1}$ \\
\hline
\end{tabular}

Fuente: Elaboración Propia.

Cálculo de la Rigidez del Resorte 


$$
K=45 *\left(\frac{W C}{W L}\right)^{2} *\left(\frac{H_{L}}{D}\right)^{2} *\left(0.5 \frac{W L}{H L}\right)
$$

Reemplazando los valores se obtiene:

$$
\mathrm{K}=259.74 \mathrm{Tn} / \mathrm{m}
$$

\subsubsection{REPORTE DE RESULTADOS}

Se realizó el modelamiento en el programa computacional SAP2000 con los datos obtenidos de los cálculos que se han mostrado, las combinaciones de cargas empleadas son las que nos brinda la norma ACI 318, ACI 371 y E060. Esta estructura y los elementos que lo conforman se diseñaron para obtener en cada una de sus secciones que las resistencias de diseño $(\varphi R n)$ por lo menos sean iguales a las resistencias requeridas $(\mathrm{Ru})$. Estas fuerzas son calculadas para las cargas y fuerzas amplificadas de acuerdo a las combinaciones de carga que se estipula en esta norma. ${ }^{28}$

Para la resistencia requerida para cargas muertas $(\mathrm{CM})$ y cargas vivas $(\mathrm{CV})$ será como mínimo:

Para el análisis se considera la carga del líquido como carga muerta.

$$
\mathrm{COMB} 1=1.4 \mathrm{CM}+1.7 \mathrm{CV}
$$

Para fuerzas de sismo (CS), la resistencia como mínimo será la siguiente, considerando la carga de la masa del agua.

$$
\begin{gathered}
\mathrm{COMB} 2=1.25(\mathrm{CM}+\mathrm{CV})+\mathrm{CS} \\
\mathrm{COMB} 3=0.9 \mathrm{CM}+\mathrm{CS}
\end{gathered}
$$

Los resultados obtenidos del programa computacional SAP2000 son los que se muestran a continuación.

${ }^{28}$ Cfr: ${ }^{28}$ ACI 318 (2014): 64 
Figura 26-III: Resultados de Combinación $=0.9 \mathrm{CM}+\mathrm{CS}$
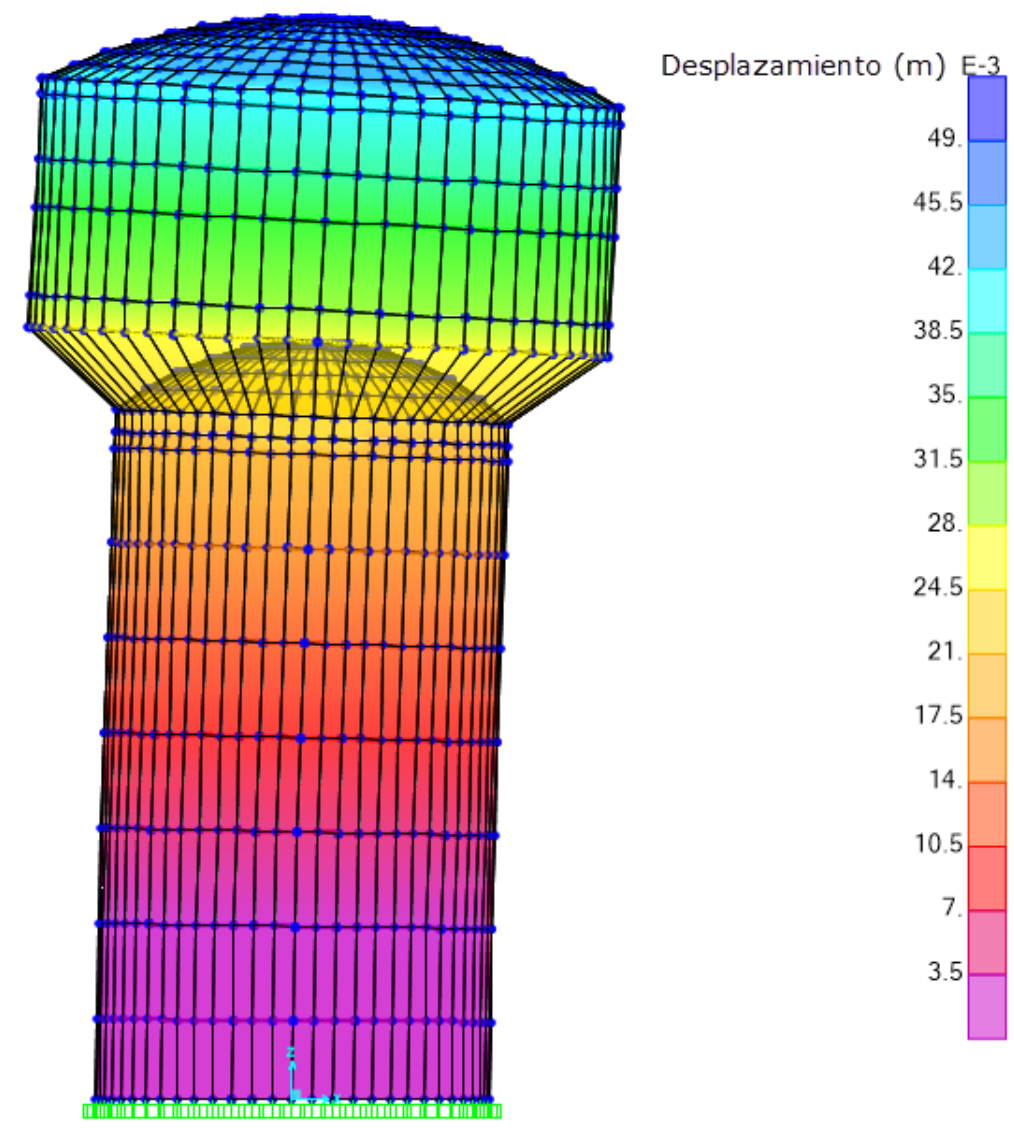

Fuente: Elaboración Propia.

En la figura 28-III se muestra idealización del tanque en el programa computacional SAP2000 para la combinación de fuerzas COMB2 $=0.9 \mathrm{CM}+\mathrm{CS}$.

Tabla 10-III: Resultados de desplazamientos máximos en el eje X (U2) para la combinación $(0.9 \mathrm{CM}+\mathrm{CS})$.

\begin{tabular}{|l|}
\hline $\begin{array}{l}\text { Desplazamiento Máximo } \\
\text { en el eje } \mathrm{x}(\mathrm{m})\end{array}$ \\
\hline 0.04180 \\
\hline
\end{tabular}

Fuente: Elaboración propia de resultados obtenidos del programa computacional SAP2000. 
Figura 27-III: Resultados de Combinaciones $=1.25(\mathrm{CM}+\mathrm{CV})+\mathrm{CS}$.
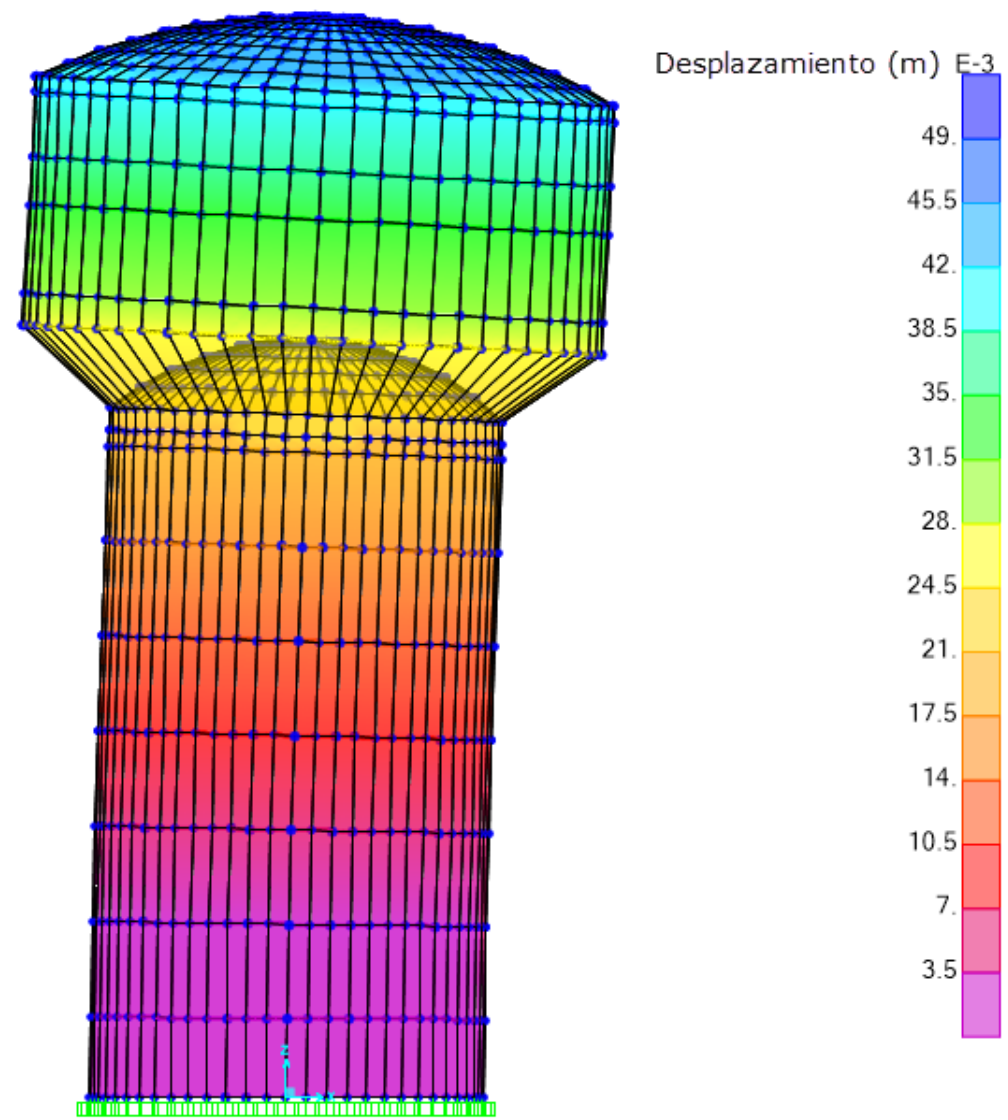

Fuente: Elaboración Propia.

En la figura 29-III se muestra idealización del tanque en el programa computacional SAP2000 para la combinación de fuerzas $1.25(\mathrm{CM}+\mathrm{CV})+\mathrm{CS}$.

Tabla 11-III: Resultados de desplazamientos máximos en el eje X (U3) para la combinación $(1.25(\mathrm{CM}+\mathrm{CV})+\mathrm{CS}$.).

\begin{tabular}{|l|}
\hline $\begin{array}{l}\text { Desplazamiento Máximo } \\
\text { en el eje } \mathrm{X}(\mathrm{m})\end{array}$ \\
\hline 0.04125 \\
\hline
\end{tabular}

Fuente: Elaboración propia de resultados obtenidos del programa computacional SAP2000.

Se muestra el desplazamiento máximo obtenido del análisis Estático. 
Tabla 12-III: Reporte de desplazamientos y análisis de resultados

\begin{tabular}{|c|c|c|}
\hline NIVEL & hi(m) & Desplaz $(\mathrm{m})$ \\
\hline VIGA SUP+CUP.SUP & 31.5 & 0.04180 \\
\hline
\end{tabular}

Fuente: Elaboración propia de desplazamientos.

\subsection{ANÁLISIS DINÁMICO}

El análisis dinámico espectral se basa en la aplicación de un espectro de respuesta, de acuerdo con una plataforma específica dependiente de la zona sísmica, uso, suelo, sistema estructural y aceleración de la gravedad. Cualquier estructura puede ser diseñada usando los resultados de los análisis dinámicos por combinación modal según lo especificado en esta norma.

Para este análisis se tiene que definir los parámetros sísmicos para obtener el espectro de pseudo aceleraciones, se hará uso de la norma ACI 350.

\section{Aceleración Espectral}

Para este análisis tenemos que definir nuestros parámetros sísmicos, luego obtenemos el espectro de seudo aceleraciones para el análisis sísmico.

Se requieren hallar los coeficientes de respuesta sísmica $(\mathrm{Ci})$ y $(\mathrm{Cc})$ para obtener el espectro de respuesta.

Coeficiente de respuesta sísmica (Ci)

Este coeficiente será determinado de acuerdo a lo siguiente:

Para $\mathrm{Ti} \leq \mathrm{Ts}:$

$$
C_{i}=S_{D S}
$$

Para Ti > Ts:

$$
C_{i}=\frac{S_{D S}}{T_{i}} \leq S_{D S}
$$

Donde 


$$
T_{S}=\frac{S_{D 1}}{S_{D S}}
$$

SDS: Es el espectro de respuesta para cortos periodos.

$$
S_{D S}=\frac{2}{3} S_{S} F_{a}
$$

$\mathrm{S}_{\mathrm{D} 1}$ : Es el espectro de respuesta para el periodo en 1 segundo.

$$
S_{D 1}=\frac{2}{3} S_{1} F_{v}
$$

$\mathrm{S}_{\mathrm{s}} \mathrm{y} \mathrm{S}_{1}$ se hallan en el mapa de isoaceleraciones espectrales (Ver Anexo)

Fa y Fv se obtienen de las tablas 12-III y 13-III; tablas 11.4-1 y 11.4-2 respectivamente de la norma ASCE 7-05.

Tabla 13-III: Coeficientes de Sitio, Fa

\begin{tabular}{|c|c|c|c|c|c|}
\hline \multirow{2}{*}{$\begin{array}{c}\text { SITE } \\
\text { CLASS }\end{array}$} & \multicolumn{6}{|c|}{$\begin{array}{c}\text { Mapped Maximum Considered Earthquake Spectral } \\
\text { Responce Acceleration Parameter at Short Period }\end{array}$} \\
\cline { 2 - 6 } & $\mathbf{S s} \leq \mathbf{0 . 2 5}$ & $\mathbf{S s}=\mathbf{0 . 5}$ & $\mathbf{S s}=\mathbf{0 . 7 5}$ & $\mathbf{S s}=\mathbf{1 . 0}$ & $\mathbf{S s} \geq \mathbf{1 . 2 5}$ \\
\hline A & 0.8 & 0.8 & 0.8 & 0.8 & 0.8 \\
\hline B & 1.0 & 1.0 & 1.0 & 1.0 & 1.0 \\
\hline C & 1.2 & 1.2 & 1.1 & 1.0 & 1.0 \\
\hline D & 1.6 & 1.4 & 1.2 & 1.1 & 1.0 \\
\hline E & 2.5 & 1.7 & 1.2 & 0.9 & 0.9 \\
\hline F & \multicolumn{5}{|c|}{ See section 11.4.7 } \\
\hline
\end{tabular}

Fuente: "Minimun Design Loads for buildings and other Structures" ASCE 7-05. p115.

Para nuestro caso, el tipo de suelo será el B debido al parámetro de suelo considerado (S1). 
Tabla 14-III: Coeficientes de Sitio, Fv

\begin{tabular}{|c|c|c|c|c|c|}
\hline \multirow{2}{*}{$\begin{array}{c}\text { SITE } \\
\text { CLASS }\end{array}$} & \multicolumn{6}{|c|}{$\begin{array}{c}\text { Mapped Maximum Considered Earthquake Spectral } \\
\text { Responce Acceleration Parameter at 1-s Period }\end{array}$} \\
\cline { 2 - 6 } & $\mathbf{S}_{\mathbf{1}} \leq \mathbf{0 . 1}$ & $\mathbf{S}_{\mathbf{1}}=\mathbf{0 . 2}$ & $\mathbf{S}_{\mathbf{1}}=\mathbf{0 . 3}$ & $\mathbf{S}_{\mathbf{1}}=\mathbf{0 . 4}$ & $\mathbf{S}_{\mathbf{1}} \geq \mathbf{0 . 5}$ \\
\hline $\mathbf{A}$ & 0.8 & 0.8 & 0.8 & 0.8 & 0.8 \\
\hline B & 1.0 & 1.0 & 1.0 & 1.0 & 1.0 \\
\hline C & 1.7 & 1.6 & 1.5 & 1.4 & 1.3 \\
\hline D & 2.4 & 2.0 & 1.8 & 1.6 & 1.5 \\
\hline E & 3.5 & 3.2 & 2.8 & 2.4 & 2.4 \\
\hline F & \multicolumn{5}{|c|}{ See section 11.4.7 } \\
\hline
\end{tabular}

Fuente: "Minimun Design Loads for buildings and other Structures" ASCE 7-05. p115.

Para nuestro caso, el tipo de suelo será el B debido al parámetro de suelo considerado (S1).

Coeficiente de respuesta sísmica $(\mathrm{Cc})$

Este coeficiente será determinado de acuerdo a lo siguiente:

Para $\mathrm{Tc} \leq 1.6 / \mathrm{Ts}$

$$
C_{C}=\frac{1.5 S_{D 1}}{T_{C}} \leq 1.5 S_{D S}
$$

Para Tc $>1.6 /$ Ts

$$
C_{C}=\frac{2.4 S_{D S}}{T_{C}{ }^{2}}
$$

De lo anterior, se obtiene:

Tabla 15-III: Resumen de Resultados

\begin{tabular}{|c|c|}
\hline $\mathrm{S} s=$ & 1.08 \\
\hline $\mathrm{S}_{1}=$ & 0.44 \\
\hline $\mathrm{F}_{\mathrm{a}}=$ & 1.00 \\
\hline $\mathrm{F}_{\mathrm{v}}=$ & 1.00 \\
\hline $\mathrm{S}_{\mathrm{D} 1}=$ & 0.293 \\
\hline $\mathrm{S}_{\mathrm{DS}}=$ & 0.720 \\
\hline $\mathrm{T}_{\mathrm{S}}=$ & 0.407 \\
\hline
\end{tabular}

Fuente: Elaboración Propia 
Tabla 16 - III: Cálculo de C para el espectro de respuesta

\begin{tabular}{|c|c|c|c|c|c|}
\hline $\mathbf{T}$ & $\mathbf{C}$ & $\mathbf{T}$ & $\mathbf{C}$ & $\mathbf{T}$ & $\mathbf{C}$ \\
\hline 0.00 & 0.7200 & 0.85 & 0.3447 & 5.50 & 0.0571 \\
\hline 0.02 & 0.7200 & 0.92 & 0.3185 & 6.00 & 0.0480 \\
\hline 0.04 & 0.7200 & 1.00 & 0.2930 & 6.50 & 0.0409 \\
\hline 0.08 & 0.7200 & 1.06 & 0.4146 & 7.00 & 0.0353 \\
\hline 0.15 & 0.7200 & 1.13 & 0.3889 & 7.50 & 0.0307 \\
\hline 0.22 & 0.7200 & 1.20 & 0.3663 & 8.00 & 0.0270 \\
\hline 0.29 & 0.7200 & 1.50 & 0.2930 & 8.50 & 0.0239 \\
\hline 0.36 & 0.7200 & 2.00 & 0.2198 & 9.00 & 0.0213 \\
\hline 0.40 & 0.7200 & 2.50 & 0.1758 & 9.50 & 0.0191 \\
\hline 0.41 & 0.7200 & 3.00 & 0.1465 & 10.00 & 0.0173 \\
\hline 0.50 & 0.5860 & 3.50 & 0.1256 & 10.50 & 0.0157 \\
\hline 0.57 & 0.5140 & 3.93 & 0.1118 & 11.00 & 0.0143 \\
\hline 0.64 & 0.4578 & 4.00 & 0.1080 & 11.50 & 0.0131 \\
\hline 0.71 & 0.4127 & 4.50 & 0.0853 & 12.00 & 0.0120 \\
\hline 0.78 & 0.3756 & 5.00 & 0.0691 & \\
\cline { 1 - 1 }
\end{tabular}

Figura 31 - III: Aceleración espectral debido a los parámetros sísmicos de la estructura.

Coeficiente de Respuesta Sísmica (C)

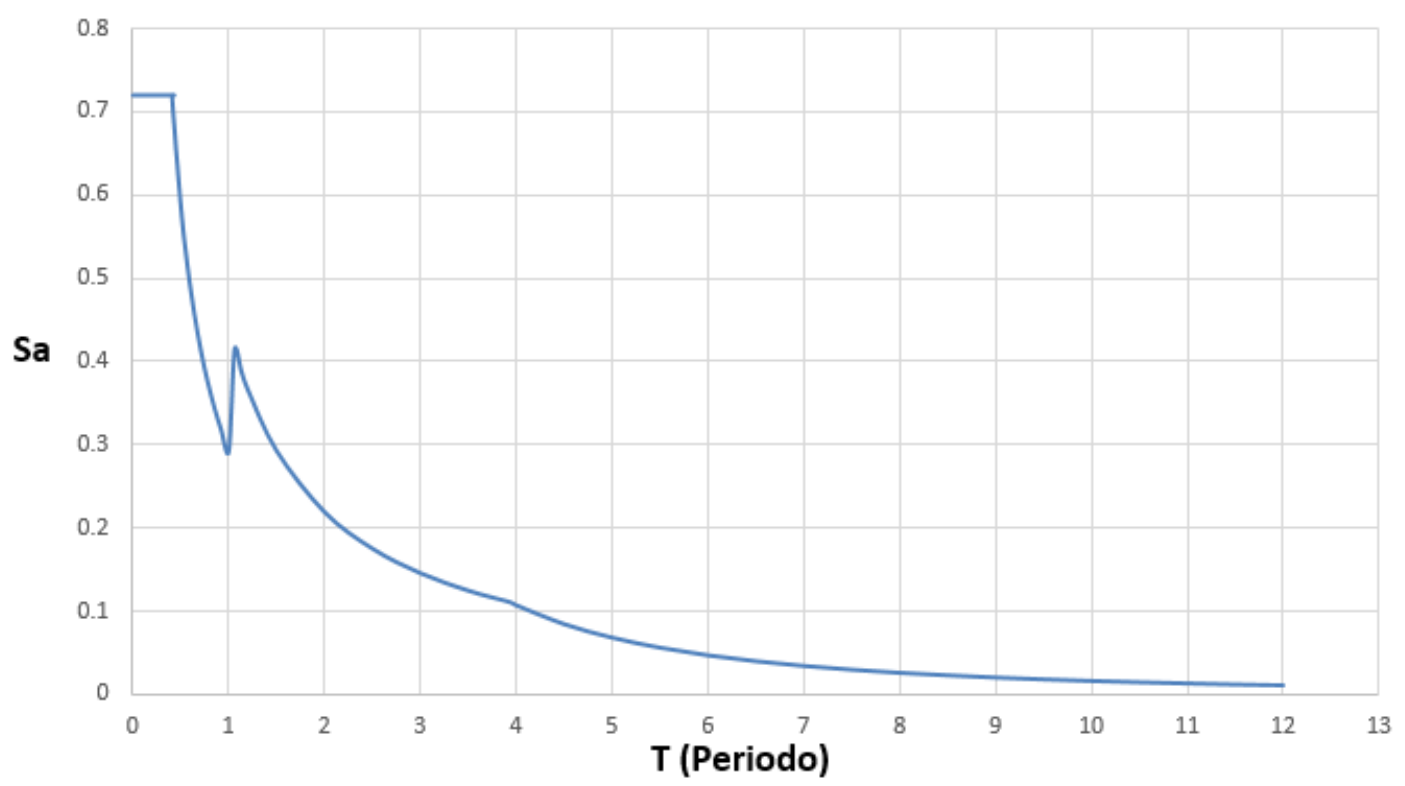

Fuente: Elaboración propia de resultados del coeficiente del suelo.

Resultados obtenidos con el programa Sap 2000:

Se consideró 150 modos de vibración: 
- Modo 2 y 3; Traslación "X" e "Y"

- Modo 9; vertical "Z"

En los criterios de combinación, se estimó con el programa mediante la combinación cuadrática completa de los valores calculados para cada modo.

Se verificará que en cada dirección se consideran aquellos modos de vibración cuya suma de masas efectivas sea por lo menos el $90 \%$ de la masa de la estructura.

SumUx representa a las masas participativas y vemos que el modo 2 alcanza más del 90\%.

Tabla 17-III: Resultados SAP2000 (Modos)

\begin{tabular}{|c|c|c|c|c|c|c|c|}
\hline OutputCase & $\begin{array}{c}\text { StepType } \\
\text { Text }\end{array}$ & $\begin{array}{l}\text { StepNum } \\
\text { Unitless }\end{array}$ & $\begin{array}{l}\text { Period } \\
\text { Sec }\end{array}$ & $\begin{array}{c}\text { UX } \\
\text { Unitless }\end{array}$ & $\begin{array}{c}\text { UY } \\
\text { Unitless }\end{array}$ & $\begin{array}{c}\text { UZ } \\
\text { Unitless }\end{array}$ & $\begin{array}{c}\text { SumUX } \\
\text { Unitless }\end{array}$ \\
\hline MODAL & Mode & 1 & 4.009965 & 0.614844 & 0 & 0 & 0.614844 \\
\hline MODAL & Mode & 2 & 0.330189 & 0.308249 & 0 & $1.04 \mathrm{E}-19$ & 0.923093 \\
\hline MODAL & Mode & 3 & 0.200563 & $4.59 \mathrm{E}-19$ & 0.657846 & 0 & 0.923093 \\
\hline MODAL & Mode & 4 & 0.084957 & $1.391 \mathrm{E}-17$ & $1.966 \mathrm{E}-15$ & $5.67 \mathrm{E}-17$ & 0.923093 \\
\hline MODAL & Mode & 5 & 0.0708 & 0.042019 & $1.698 \mathrm{E}-15$ & $2.565 E-14$ & 0.965112 \\
\hline MODAL & Mode & 6 & 0.07029 & 1.096E-16 & 0.17896 & $2.957 \mathrm{E}-16$ & 0.965112 \\
\hline MODAL & Mode & 7 & 0.07029 & 1.7E-17 & $1.042 \mathrm{E}-12$ & $2.019 \mathrm{E}-16$ & 0.965112 \\
\hline MODAL & Mode & 8 & 0.07029 & $8.365 \mathrm{E}-16$ & 4.408E-11 & $6.609 \mathrm{E}-16$ & 0.965112 \\
\hline MODAL & Mode & 9 & 0.061504 & $2.945 \mathrm{E}-17$ & $2.08 \mathrm{E}-15$ & 0.546947 & 0.965112 \\
\hline MODAL & Mode & 10 & 0.060964 & $1.077 \mathrm{E}-15$ & $2.292 \mathrm{E}-15$ & $3.46 \mathrm{E}-16$ & 0.965112 \\
\hline MODAL & Mode & 11 & 0.060964 & $2.527 \mathrm{E}-15$ & $1.45 \mathrm{E}-15$ & $3.724 \mathrm{E}-14$ & 0.965112 \\
\hline MODAL & Mode & 12 & 0.052683 & $3.414 \mathrm{E}-14$ & $1.773 \mathrm{E}-13$ & $6.491 \mathrm{E}-13$ & 0.965112 \\
\hline MODAL & Mode & 13 & 0.052683 & $2.182 \mathrm{E}-16$ & $2.631 \mathrm{E}-14$ & $1.212 \mathrm{E}-13$ & 0.965112 \\
\hline MODAL & Mode & 14 & 0.05021 & 0.000977 & $1.937 \mathrm{E}-16$ & $1.394 \mathrm{E}-13$ & 0.966089 \\
\hline MODAL & Mode & 15 & 0.04059 & $2.374 \mathrm{E}-16$ & $1.411 \mathrm{E}-17$ & 0.105814 & 0.966089 \\
\hline
\end{tabular}

Fuente: Elaboración propia de resultados obtenidos del programa computacional SAP2000.

RESULTADOS:

Periodos por Modos: 


\begin{tabular}{|l|l|}
\cline { 2 - 2 } \multicolumn{1}{c|}{} & $\mathrm{T}(\mathrm{seg})$ \\
\hline Modo 1 & 4.0099 \\
\hline Modo 2 & 0.3302 \\
\hline Modo 3 & 0.2001 \\
\hline Modo 4 & 0.0850 \\
\hline Modo 5 & 0.0708 \\
\hline Modo 6 & 0.0703 \\
\hline Modo 7 & 0.0703 \\
\hline Modo 8 & 0.0703 \\
\hline
\end{tabular}

Cortante en la base:

Tabla 18-III: Cortante en la base (Dinámico)

\begin{tabular}{|c|c|}
\hline Cortante & Ton-f \\
\hline Vdin & 1183.15 \\
\hline
\end{tabular}

De acuerdo a la norma E030 el valor de la fuerza estática deberá ser menor a un $90 \%$ de la fuerza dinámica.

Vdin $>90 \%$ Vestatico (No Cumple)

Se tendrá el siguiente factor de escala.

$$
\begin{gathered}
F e=\frac{0.9(\text { Vest })}{V d i n} \\
\mathrm{Fe}=1.3
\end{gathered}
$$

Dando como cortante en la base:

Tabla 19-III: Cortante en la base (Dinámico)

\begin{tabular}{|c|c|}
\hline Cortante & Ton-f \\
\hline Vdin & 1496.68 \\
\hline
\end{tabular}

Desplazamiento máximo:

Tabla 20-III: Reporte de Desplazamientos para el estado Dinámico

\begin{tabular}{|c|c|r|}
\hline NIVEL & hi(m) & Desplaz (m) \\
\hline VIGA SUP+CUP.SI & 31.50 & 0.0356 \\
\hline
\end{tabular}




\subsubsection{Espectro Dinámico con Factor de Escala}

Con el factor de escala, se modifica el modelo en SAP2000 y se tiene los siguientes modos de vibración de la estructura,

Tabla 21: Resultados SAP2000 (Modos) - Con Factor de Escala

\begin{tabular}{|l|l|r|r|r|r|r|r|}
\hline OutputCase & $\begin{array}{c}\text { StepType } \\
\text { Text }\end{array}$ & \multicolumn{1}{c|}{$\begin{array}{c}\text { StepNum } \\
\text { Unitless }\end{array}$} & \multicolumn{1}{c|}{$\begin{array}{c}\text { Period } \\
\text { Sec }\end{array}$} & $\begin{array}{c}\text { UX } \\
\text { Unitless }\end{array}$ & $\begin{array}{c}\text { UY } \\
\text { Unitless }\end{array}$ & \multicolumn{1}{c}{$\begin{array}{c}\text { UZ } \\
\text { Unitless }\end{array}$} & \multicolumn{1}{c}{$\begin{array}{c}\text { SumUX } \\
\text { Unitless }\end{array}$} \\
\hline MODAL & Mode & 1 & 4.01551 & 0.627822 & 0 & 0 & 0.627822 \\
\hline MODAL & Mode & 2 & 0.355303 & 0.306075 & $5.024 \mathrm{E}-20$ & $3.451 \mathrm{E}-20$ & 0.933896 \\
\hline MODAL & Mode & 3 & 0.215529 & $8.88 \mathrm{E}-20$ & 0.680754 & $1.015 \mathrm{E}-20$ & 0.933896 \\
\hline MODAL & Mode & 4 & 0.091412 & $5.201 \mathrm{E}-16$ & $1.169 \mathrm{E}-14$ & $9.621 \mathrm{E}-16$ & 0.933896 \\
\hline MODAL & Mode & 5 & 0.075278 & $1.123 \mathrm{E}-16$ & $7.482 \mathrm{E}-17$ & $7.686 \mathrm{E}-16$ & 0.933896 \\
\hline MODAL & Mode & 6 & 0.075278 & $1.429 \mathrm{E}-16$ & $2.705 \mathrm{E}-15$ & $4.684 \mathrm{E}-16$ & 0.933896 \\
\hline MODAL & Mode & 7 & 0.072136 & 0.036194 & $3.444 \mathrm{E}-15$ & $1.305 \mathrm{E}-14$ & 0.97009 \\
\hline MODAL & Mode & 8 & 0.07179 & $1.401 \mathrm{E}-17$ & 0.169473 & $1.235 \mathrm{E}-15$ & 0.97009 \\
\hline MODAL & Mode & 9 & 0.063616 & $2.356 \mathrm{E}-18$ & $5.412 \mathrm{E}-16$ & 0.605806 & 0.97009 \\
\hline MODAL & Mode & 10 & 0.061086 & $2.29 \mathrm{E}-16$ & $7.838 \mathrm{E}-15$ & $2.25 \mathrm{E}-14$ & 0.97009 \\
\hline MODAL & Mode & 11 & 0.061086 & $6.28 \mathrm{E}-17$ & $5.487 \mathrm{E}-17$ & $8.45 \mathrm{E}-16$ & 0.97009 \\
\hline MODAL & Mode & 12 & 0.060886 & $5.567 \mathrm{E}-17$ & $3.473 \mathrm{E}-16$ & $2.132 \mathrm{E}-17$ & 0.97009 \\
\hline MODAL & Mode & 13 & 0.060886 & $3.168 \mathrm{E}-16$ & $5.891 \mathrm{E}-17$ & $3.629 \mathrm{E}-15$ & 0.97009 \\
\hline MODAL & Mode & 14 & 0.050104 & 0.000945 & $7.961 \mathrm{E}-15$ & $3.033 \mathrm{E}-13$ & 0.971035 \\
\hline MODAL & Mode & 15 & 0.042123 & $5.307 \mathrm{E}-15$ & $2.013 \mathrm{E}-15$ & $3.144 \mathrm{E}-15$ & 0.971035 \\
\hline
\end{tabular}

Fuente: Elaboración propia de resultados obtenidos del programa computacional SAP2000.

\subsubsection{Verificación de Periodos}

Es necesario realizar un cálculo de los periodos de vibración por lo cual nos basamos en los cálculos matriciales con el modelo equivalente de dos masas. 
Figura 32-III: Modelo de masas equivalentes.

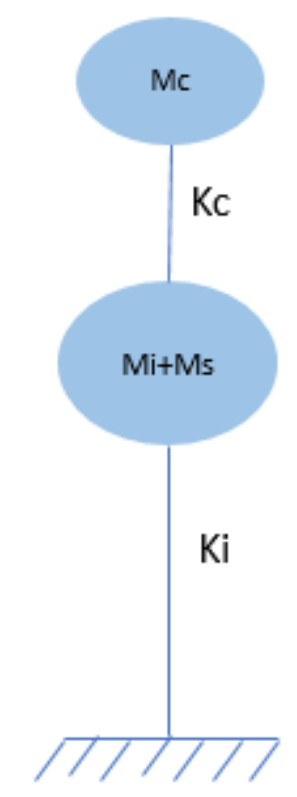

Fuente: Elaboración Propia.

Se tiene que:

$$
\left|\mathrm{K}-\omega^{2} M\right|=0
$$

Donde:

K : Matriz de Rigidez.

$\omega:$ Frecuencia angular

M: Matriz de masas

$\mathrm{M} 1: \mathrm{Mi}+\mathrm{Ms}$

Mi: Masa impulsiva.

Ms: Masa de la estructura.

M2: Masa convectiva (Mc).

K1: Rigidez de la masa convectiva (Kc).

K2: Rigidez de la masa impulsiva (Ki). 
Para la rigidez lateral se tiene la siguiente:

$$
K s=\frac{3 E_{c} I_{f}}{h_{f}^{3}}
$$

Donde:

$$
I_{f}=\pi\left[\frac{\left(D_{f}+2 t_{f}\right)^{4}-D_{f}^{4}}{64}\right]
$$

Df: diámetro del fuste

tf:: espesor del fuste

$\mathrm{I}_{\mathrm{f}}=247.20 \mathrm{~m}^{\wedge} 4$

$\mathrm{Ks}=200994.05 \mathrm{Tn} / \mathrm{m}$

Por lo tanto, se tiene la siguiente matriz:

$$
\left|\left[\begin{array}{cc}
K 1+K 2 & -K 2 \\
-K 2 & K 2
\end{array}\right]-\omega^{2}\left[\begin{array}{cc}
M 1 & 0 \\
0 & M 2
\end{array}\right]\right|=0
$$

Resolviendo, se obtiene:

$$
\omega^{2}=\frac{\left(K_{1} M_{2}+K_{2} M_{1}+K_{2} M_{2}\right) \pm \sqrt{\left(K_{1} M_{2}+K_{2} M_{1}+K_{2} M_{2}\right)^{2}-4 K_{1} K_{2} M_{1} M_{2}}}{2 M_{1} M_{2}}
$$

$\omega_{1}=1.58 \mathrm{rad} / \mathrm{s}$

$\omega_{2}=32.71 \mathrm{rad} / \mathrm{s}$

De los cálculos se calcula el periodo fundamental de la estructura en su primer modo:

$\mathrm{T}_{1}=3.98 \mathrm{~s} ;$ Periodo fundamental de la estructura

La cual se verifica con la tabla $\mathrm{N}^{\circ} 20$, la cual tiene una aceleración en el primer modo de 4.01 S. 


\subsubsection{Verificación de Desplazamientos}

La verificación de desplazamientos para este tipo de estructuras se realizó considerando la norma peruana E030-2018, la norma ASCE 7-05 y la guía IITK-GSDMA.

Para el E030, en la Tabla $\mathrm{N}^{\circ} 11$, se establece límites de desplazamientos relativo de entrepiso $(\Delta)$, con lo cual se ha verificó la deriva de la estructura:

Tabla 22: Límites para la distorsión de entrepiso.

\begin{tabular}{|l|c|}
\hline Mate rial Predominante & $\left(\Delta \mathbf{i} / \mathbf{h}_{\mathrm{ei}}\right)$ \\
\hline Concreto Armado & 0.007 \\
\hline Acero & 0.010 \\
\hline Albañilería & 0.005 \\
\hline Madera & 0.010 \\
\hline $\begin{array}{l}\text { Edificios de concreto } \\
\text { armado con muros de } \\
\text { ductilidad limitada }\end{array}$ & 0.005 \\
\hline
\end{tabular}

Fuente: “Diseño Sismo resistente” E030-2018.

\begin{tabular}{|l|c|c|c|}
\hline \multicolumn{1}{|c|}{ NIVEL } & Desplaz $(\mathrm{m})$ & Deriva & Cumple \\
\hline VIGA SUP+CUP.SUP & 0.0418 & 0.0014 & SI \\
\hline CONVECTIVA & 0.0405 & 0.0009 & SI \\
\hline IMPULSIVO & 0.0306 & 0.0008 & SI \\
\hline CUB+VIG+CHIM & 0.0275 & 0.0009 & SI \\
\hline CUP.INF+TRONC+VIG & 0.0240 & 0.0011 & SI \\
\hline FUSTE 7 & 0.0183 & 0.0019 & SI \\
\hline FUSTE 6 & 0.0146 & 0.0014 & SI \\
\hline FUSTE 5 & 0.0110 & 0.0010 & SI \\
\hline FUSTE 4 & 0.0079 & 0.0009 & SI \\
\hline FUSTE 3 & 0.0051 & 0.0007 & SI \\
\hline FUSTE 2 & 0.0030 & 0.0006 & SI \\
\hline FUSTE 1 & 0.0013 & 0.0005 & SI \\
\hline
\end{tabular}

En el ASCE 7, en el capítulo 9.14.6.5.1, recomienda que la verificación para los desplazamientos del tanque elevado se realice considerando el efecto P-delta.

Efecto P-Delta: 
Para considerar el efecto P-Delta se requiere que el coeficiente de estabilidad de la estructura $\theta \geq 0.1$.

Se realizará el cálculo del coeficiente de estabilidad para el tanque elevado según la ecuación Ecuación 9.5.5.7.2.-1- ASCE 7- 05.

$$
\theta=\frac{P_{x} \Delta}{V_{x} h_{s x} C_{d}}
$$

Donde:

Cd: Para tanques elevados $=2$

hsx: Altura de la estructura portante

Px: Carga vertical total

$\Delta$ : Desplazamiento

Vx: Fuerza cortante

Se obtiene:

$\begin{array}{lll}\Delta= & 0.0183 & \mathrm{~m} \\ \mathrm{hsx}= & 21 & \mathrm{~m} \\ \mathrm{Px}= & 2666.45 & \mathrm{Tn} \\ \mathrm{Vx}= & 1602.00 \quad \mathrm{Tn} \\ \mathrm{Cd}= & 2 & \\ \boldsymbol{\theta}= & \mathbf{0 . 0 0 0 7} \leq \mathbf{0 . 1}\end{array}$

Por lo tanto, no se requiere el análisis de segundo orden para la estructura.

La guía de diseño sísmico para estructuras contenedoras de líquido IITK-GSDMA indica en el capítulo 4.13.5 que el efecto P-delta puede ser minimizado restringiendo el desplazamiento 
lateral, se recomienda que en los reservorios el efecto P-delta sea mínimo o despreciable para lo cual se da 1 :

$$
\Delta=\frac{h_{f}}{500}
$$

$\Delta=$ desplazamiento lateral $(\mathrm{m})=0.042 \mathrm{~m}$.

$\mathrm{hf}=$ altura de la estructura portante.

\subsection{ESFUERZO DE VON MISES}

La teoría de Von Mises propone que los materiales bajo esfuerzos hidrostáticos soportan esfuerzos mucho mayores que sus esfuerzos de fluencia bajo otros estados de carga. La teoría establece:

"La falla se producirá cuando la energía de distorsión por unidad de volumen debida a los esfuerzos máximos absolutos en el punto crítico sea igual o mayor a la energía de distorsión por unidad de volumen de una probeta en el ensayo de tensión en el momento de producirse la fluencia". ${ }^{29}$

Figura 28-III: Esfuerzo máximo que actúa en la Cuba

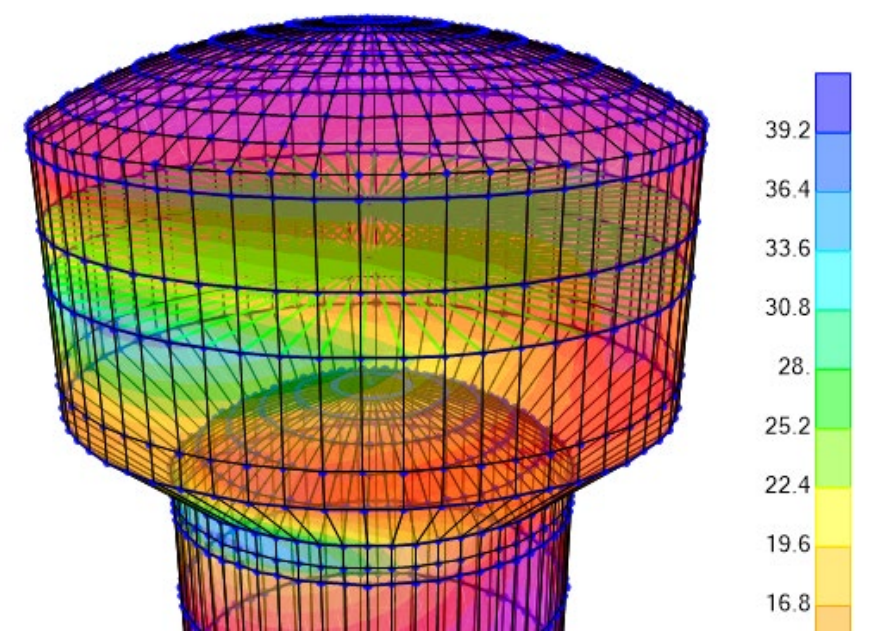

Fuente: Elaboración Propia

${ }^{29}$ Cfr: De Castro (2013):3 
Tabla 23-III: Resultados SAP2000 (Esfuerzo máximo)

\begin{tabular}{|c|c|l|l|l|l|l|r|}
\hline $\begin{array}{l}\text { Area } \\
\text { Text }\end{array}$ & AreaElem & ShellType & $\begin{array}{c}\text { Joint } \\
\text { Text }\end{array}$ & utputCas & CaseType & StepType & $\begin{array}{c}\text { S11Top } \\
\text { Kgf/cm2 }\end{array}$ \\
\hline 1262 & 1262 & Shell-Thi... & 2372 & SPX & LinResp... & Max & 36.92 \\
\hline 1289 & 1289 & Shell-Thi... & 2400 & SPX & LinResp... & Max & 36.92 \\
\hline 1292 & 1292 & Shell-Thi... & 2402 & SPX & LinResp... & Max & 36.92 \\
\hline 1319 & 1319 & Shell-Thi... & 2430 & SPX & LinResp... & Max & 36.92 \\
\hline 1261 & 1261 & Shell-Thi... & 2185 & SPX & LinResp... & Max & 36.77 \\
\hline 1290 & 1290 & Shell-Thi... & 2401 & SPX & LinResp... & Max & 36.77 \\
\hline 1291 & 1291 & Shell-Thi... & 2401 & SPX & LinResp... & Max & 36.77 \\
\hline 1320 & 1320 & Shell-Thi... & 2185 & SPX & LinResp... & Max & 36.77 \\
\hline
\end{tabular}

$\sigma_{c}=2 \sqrt{f^{\prime} c} \quad ;$ Esfuerzo del concreto, se considerará f'c $=350 \mathrm{~kg} / \mathrm{cm} 2$

$\mathrm{FVM}=369.20 \mathrm{Ton} / \mathrm{m} 2$

$\mathrm{FVM}=36.92 \mathrm{~kg} / \mathrm{cm} 2$

$\sigma \mathrm{c}=37.416 \mathrm{~kg} / \mathrm{cm} 2$

Se cumple que el FVM no excede el esfuerzo del concreto.

\subsection{CÁLCULO DEL REFUERZO}

Para el cálculo del refuerzo de cada uno de los elementos del tanque se debe tener en cuenta lo siguiente:

\subsubsection{Refuerzo por corte}

Es el cálculo del refuerzo de las secciones transversales de los elementos sometidos a fuerza cortante. Se tiene lo siguiente:

$$
\emptyset V_{s} \geq S_{d}\left(V_{U}-\emptyset V_{C}\right)
$$

Sd es el factor de durabilidad en la sección C9.2.9 del ACI 350-06, indica que para fuerzas cortantes que toma el acero de refuerzo esta equivale a 1.30.

La contribución del concreto Vc se podrá evaluar para miembros únicamente a corte y flexión como: 


$$
V_{c} \geq 0.53 \sqrt{f^{\prime} c} . b w . d
$$

Donde:

bw: ancho de viga

d: peralte efectivo

Para vigas de concreto armado:

$$
\emptyset V_{s}=\frac{A v \cdot f y \cdot d}{S} \geq V u-\emptyset V c
$$

Se tiene el espaciamiento de estribos como:

$$
S \geq \frac{A v \cdot f y \cdot d}{V u-\emptyset V c}
$$

Donde:

Av: es el área de refuerzo por cortante dentro de una distancia "S" proporcionada en la ecuación 54.

Vu: es la resistencia al corte en la sección analizada

Vc: resistencia al corte del concreto.

\subsubsection{Refuerzo por Tracción Anular}

Para elementos a tracción pura, tales como las vigas inferior y superior y la cuba.

$$
\varnothing T_{u}=\emptyset f y . A s \geq T u
$$

Obteniendo:

$$
A s=\frac{T u}{\emptyset \cdot f y}
$$

Donde:

Tu: Fuerza cortante ultima 


\subsubsection{Refuerzo por Compresión en muros de carga}

Para muros sujetos a cargas de compresión o flexo-compresión, se diseñará el refuerzo mediante la siguiente formula empírica.

$$
\emptyset P_{n}=0.55 \emptyset f^{\prime} c . A g\left[1-\left(\frac{k \cdot l_{c}}{32 h}\right)\right]
$$

Donde:

Pn: Resistencia de carga axial nominal de pared.

lc: distancia vertical entre apoyos.

h: espesor del elemento

Ag: área bruta de la sección del muro.

La norma considera las siguientes cuantías mínimas de refuerzo, para barras corrugadas, con relación a la sección bruta del elemento.

Refuerzo vertical:

- 0.0012 para barras $\phi \leq 5 / 8$ "

- 0.0015 para otros diámetros

Refuerzo horizontal

- 0.0020 para barras $\phi \leq 5 / 8 ”$

- 0.0025 para otros diámetros

\subsubsection{Diseño por Flexión}

El diseño por flexión debe cumplir la condición reglamentaria la cual establece que la resistencia a flexión de una sección de concreto reforzado debe tener una magnitud que exceda o cuando menos sea igual a la del momento último producido por las cargas, es decir

$$
\mathrm{Mr}>\mathrm{Mu}
$$


Figura 29: Deformación del acero $\square$ t para flexión positiva

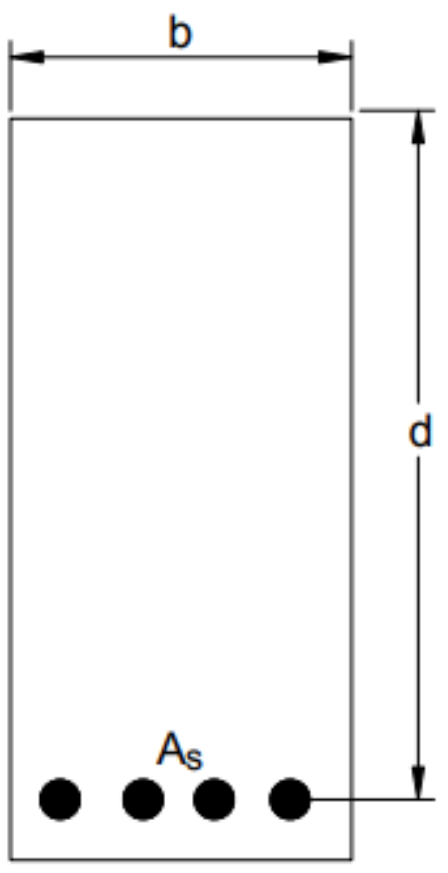

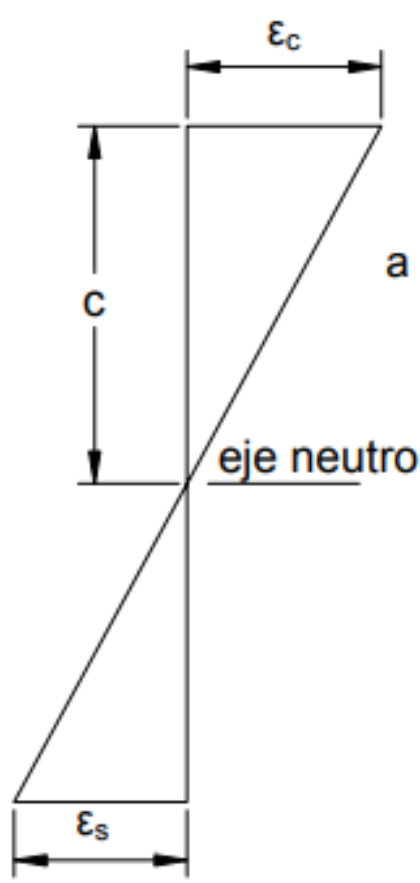

Deformación

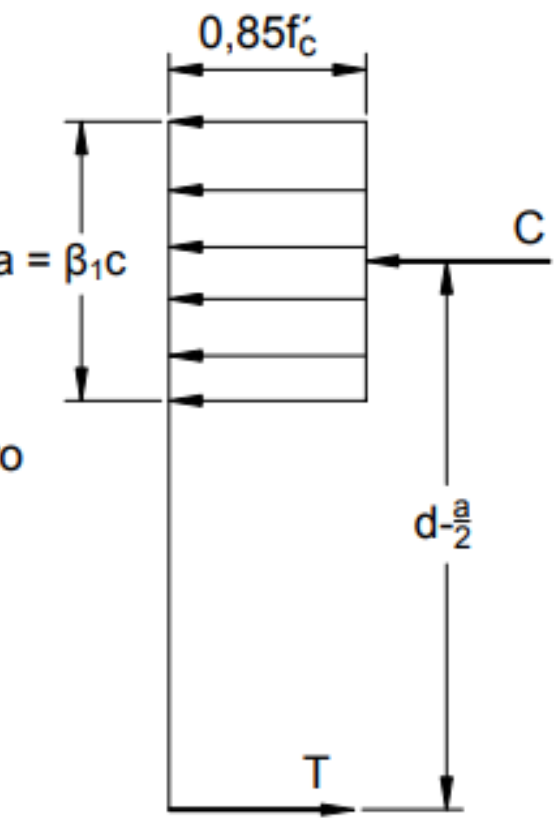

Tensión equivalente

Fuente: "Norma técnica - E060" - pagina61

Equilibrio de Fuerzas de compresión y tracción

$$
\begin{gathered}
\mathrm{C}=\mathrm{T} \\
\text { 0.85.f'c.b.a=As.fy=o.b.d.fy } \\
a=\frac{A_{s} \cdot f_{y}}{0,85 \cdot f^{\prime} \cdot b}=\frac{\rho \cdot d \cdot f_{y}}{0,85 \cdot f^{\prime}{ }_{c}}
\end{gathered}
$$

Por equilibrio de momentos

$$
M_{n}=\rho . \text { b.d. }\left[d-\frac{0.5 * \rho \cdot d \cdot f_{y}}{0,85 \cdot f^{\prime}{ }_{c}}\right]
$$

Se define:

$$
\omega=\rho \cdot \frac{f_{y}}{f^{\prime}{ }_{c}}
$$


Dividiendo por $b^{2}$, se obtiene:

$$
\rho=\frac{0,85 \cdot f^{\prime} c}{f y} \cdot\left(\frac{1-\sqrt{1-4 \cdot 0,59 \cdot K u}}{2 \cdot 0,59}\right)
$$

\subsubsection{Espaciamiento Máximo Por Fisuramiento}

Para prevenir fugas y garantizar la impermeabilidad en los elementos contenedores de líquido, el ACI recomienda controlar las fisuras, esto se consigue proporcionando una adecuada distribución del acero principal, verificando un espaciamiento máximo "S":

$$
\begin{gathered}
S \max \leq \frac{z^{3}}{2 \cdot d c^{2} \cdot f s} \\
Z=f s \cdot \sqrt[3]{d c \cdot A}
\end{gathered}
$$

Donde:

$\mathrm{Z}$ : factor en $\mathrm{kgf} / \mathrm{cm}$, según el grado de exposición y ancho de grieta. $(Z=16900 \mathrm{kgf} / \mathrm{cm}$ para exposición severa ACI350-01)

S: separación de varillas $(\mathrm{cm})$

A: área de la sección efectiva a tracción.

fs: Tensión admisible del acero

dc: recubrimiento del concreto medido desde la fibra extrema de tensión, al eje del refuerzo principal. 
Figura 30: Área efectiva de la zona a tracción

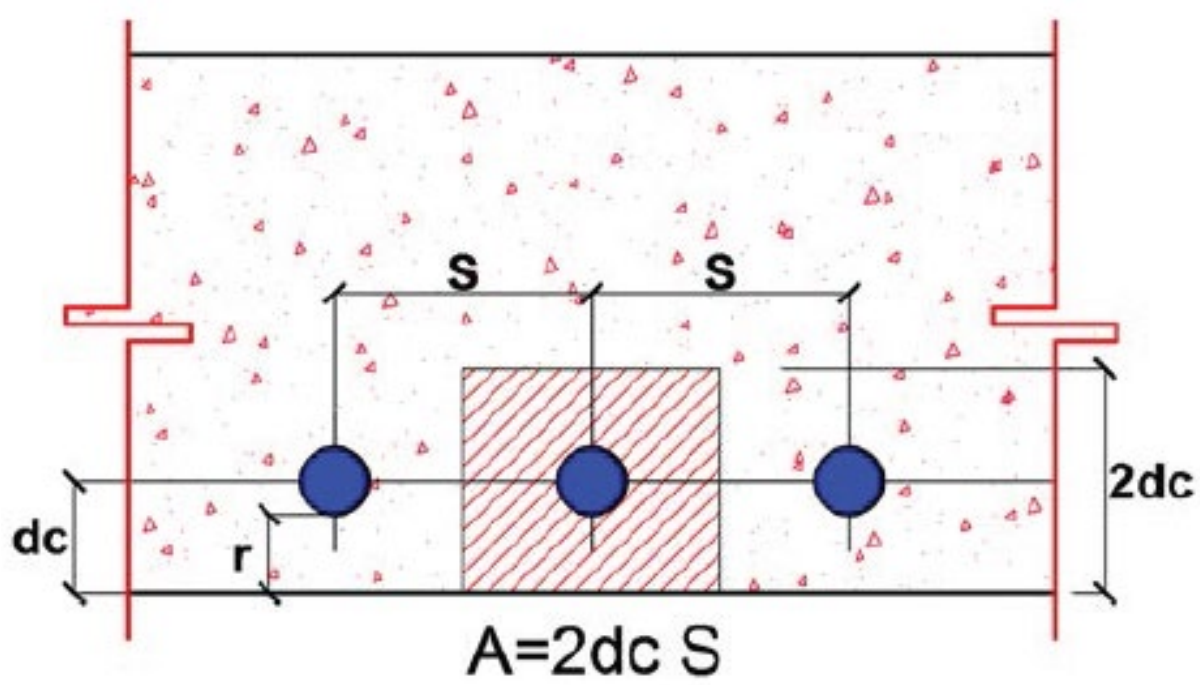

Fuente: "Diseño Estructural de Tanques y sus aplicaciones" Pag-4

\subsubsection{Espesor mínimo de Muros}

Para el cálculo del espesor mínimo de cada una de las paredes de la estructura, nos basamos en PCA. La cual indica que cualquier agrietamiento significativo en un tanque contenedor de líquido es inaceptable. Por lo cual adopta la siguiente formula basándose en los esfuerzos de tensión del concreto y del acero.

$$
t=\frac{\varepsilon s h \cdot E s+f_{s}-n f_{c t}}{b f_{s} f_{c t}} . T
$$

$\varepsilon s h=0.0003 ;$ Coeficiente de contracción del concreto armado (PCA).

$\mathrm{Es}=2.1 \mathrm{x} 10 \mathrm{E}+6 \mathrm{~kg} / \mathrm{cm} 2 ;$ Módulo de elasticidad del acero.

$\mathrm{f}^{\prime} \mathrm{c}=350 \mathrm{~kg} / \mathrm{cm} 2$

$\mathrm{Ec}=2.8 \times 10 \mathrm{E}+5 \mathrm{~kg} / \mathrm{cm} 2 ;$ Módulo de elasticidad del concreto

$\mathrm{fs}=1400 \mathrm{~kg} / \mathrm{cm} 2$; Tensión admisible del acero.

fct $=0.1 f^{\prime}$ c; Esfuerzo permisible del concreto a tensión.

$\mathrm{n}=\mathrm{Es} / \mathrm{Ec}=7.48$ 
$\mathrm{T}=$ Tensión en el elemento.

\subsection{DISEÑO ESTRUCTURAL DE ELEMENTOS}

El objetivo del diseño consiste en determinar las dimensiones y características de los elementos que constituyen la estructura para que puedan resistir adecuadamente las cargas para las que han sido diseñadas, y con un grado de seguridad razonable.

Se diseñará esta estructura usando el estado límite último de rotura o agotamiento, se dice que una estructura o elemento estructural ha alcanzado un estado límite cuando ésta no es capaz de cumplir alguna de las funciones para las que ha sido diseñada. El estado límite último de rotura o agotamiento involucra el colapso total o parcial de la estructura.

La primera condición para el diseño de la estructura es que la estructura sea lo suficientemente segura, esto es, evitar que la estructura alcance o sobrepase el estado límite ya descrito. Las normas actuales de concreto armado se centran en el estado límite último por rotura o agotamiento, también llamado Diseño por Resistencia, de las secciones que conforman la estructura. El factor de seguridad se puede definir como el cociente entre la resistencia y el valor estimado de la solicitación correspondiente en condiciones de servicio. La evaluación cuantitativa del factor de seguridad requiere comparar la demanda de resistencia con la resistencia máxima.

Entonces, la seguridad de la estructura podría garantizarse suministrando una resistencia ligeramente superior a la demanda por carga.

Para el diseño de esta estructura se usan las combinaciones de carga que dicta la 371R-11 en la sección 5.1.2:

$$
\begin{gathered}
\mathrm{U}=1.4 \mathrm{CM}+1.7 \mathrm{CV} \\
\mathrm{U}=1.2(\mathrm{CM}+\mathrm{CL})+1.6 \mathrm{CV} \\
\mathrm{U}=1.2(\mathrm{CM}+\mathrm{CL})+1.0 \mathrm{CS}+0.5 \mathrm{CV} \\
\mathrm{U}=0.9 \mathrm{CM}+1.0 \mathrm{CL}+1.0 \mathrm{CS}
\end{gathered}
$$

Para este análisis se añadió el efecto de la masa del agua CL, también considerada en el análisis computacional (SAP200). 
Las fuerzas internas en el elemento Shell como resultado del modelamiento en el programa computacional SAP2000 se muestran en las siguientes figuras 33-III, 34-III, 35-III:

Figura 31- III: Fuerzas en el elemento Shell

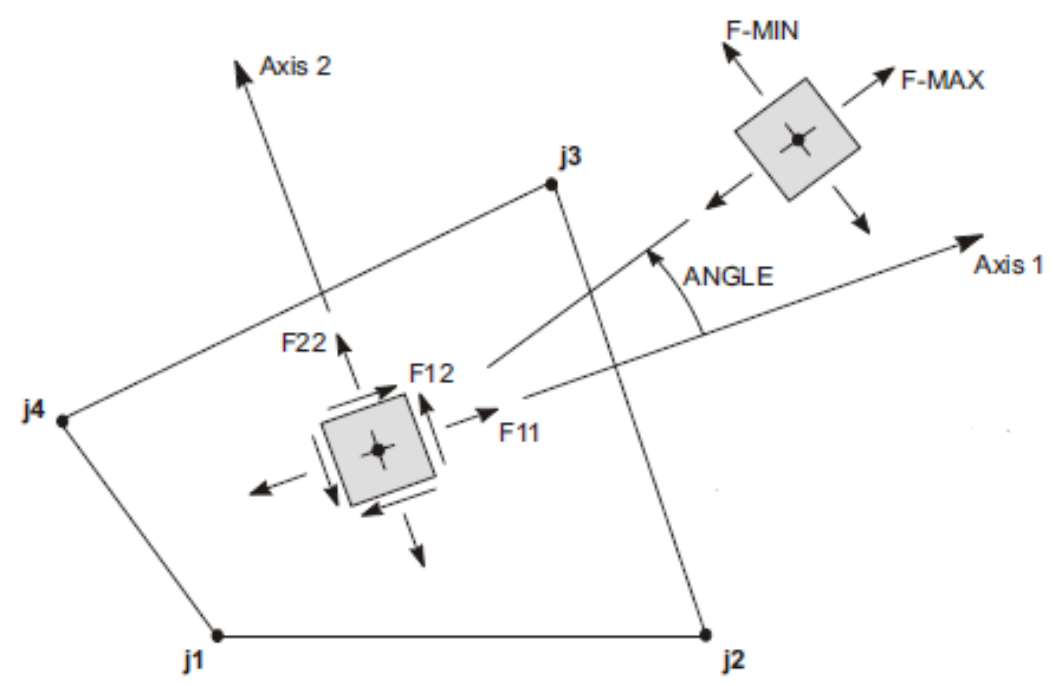

Fuente: “CSI Analysis Reference Manual”, p233

Figura 32- III: Momentos en el elemento Shell

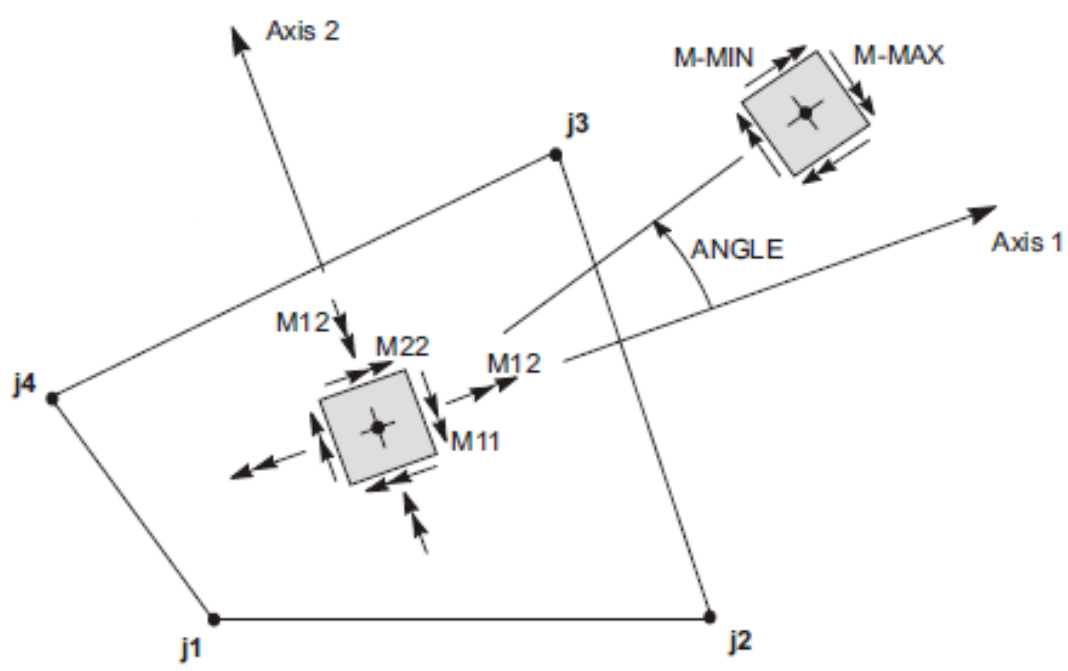

Fuente: “CSI Analysis Reference Manual”, p233

Para las cortantes, se muestra la figura 35-III tridimensional donde: 
Figura 33- III: Idealización para fuerzas cortantes

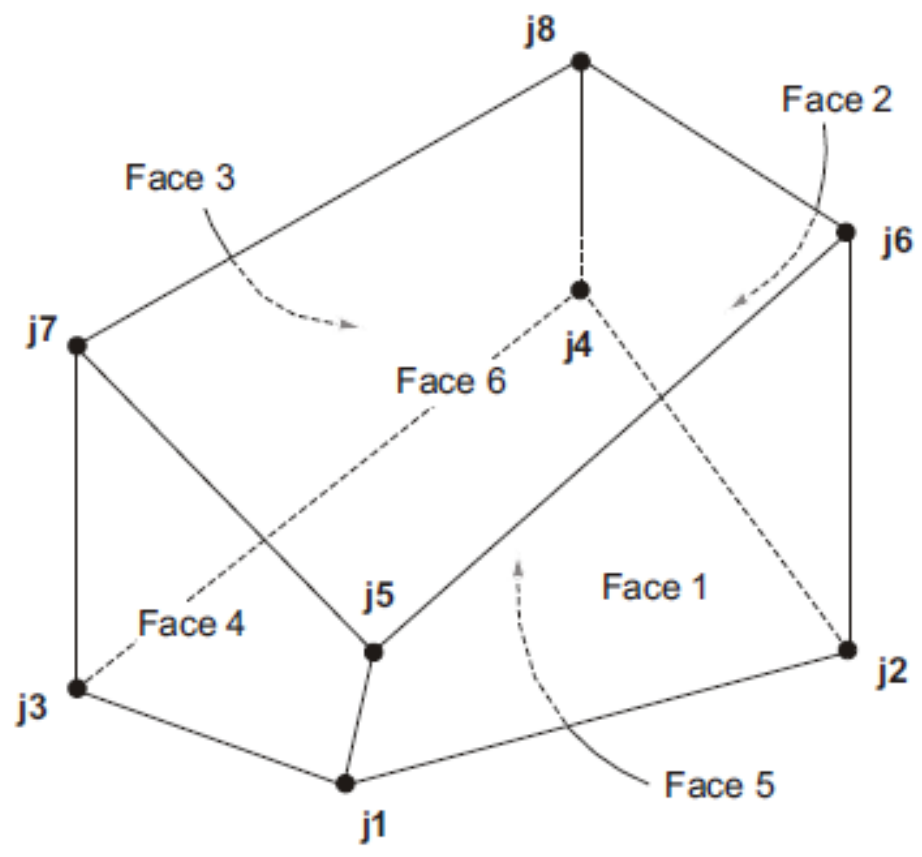

Fuente: “CSI Analysis Reference Manual”, p234

V12: De j1 a j2

V13: de j1 a j3

\subsubsection{DISEÑO DE CÚPULA SUPERIOR}

La tapa del tanque elevado (casquete esférico), tiene la característica de estar apoyado únicamente en su periferia, tiene que ser calculado y diseñado para que su forma geométrica, en combinación con el espesor mínimo requerido, absorba la carga generada por su propio peso más las cargas vivas. La cúpula elemento que está sometido a esfuerzos de compresión en toda su área salvo en el sector de la unión con las paredes del tanque donde existe esfuerzos de tracción, para el diseño se verifica que el concreto resista el esfuerzo a compresión generado y el refuerzo de acero mínimo colocado absorba el esfuerzo de tracción.

Diseño por membrana lineal con valores obtenidos del análisis en el programa computacional SAP2000.

La teoría de falla de máxima energía de distorsión se presenta en materiales dúctiles que son capaces de absorber una cierta cantidad de energía antes de sufrir una falla. La teoría establece 
que la falla ocurrirá cuando la energía de distorsión por volumen unitario exceda el esfuerzo de compresión en la falla, es decir, no exceda el esfuerzo del concreto $\sigma c=2 \cdot \sqrt{f^{\prime} c}$

Figura 39-III: Fuerzas actuantes F22 Dirección X
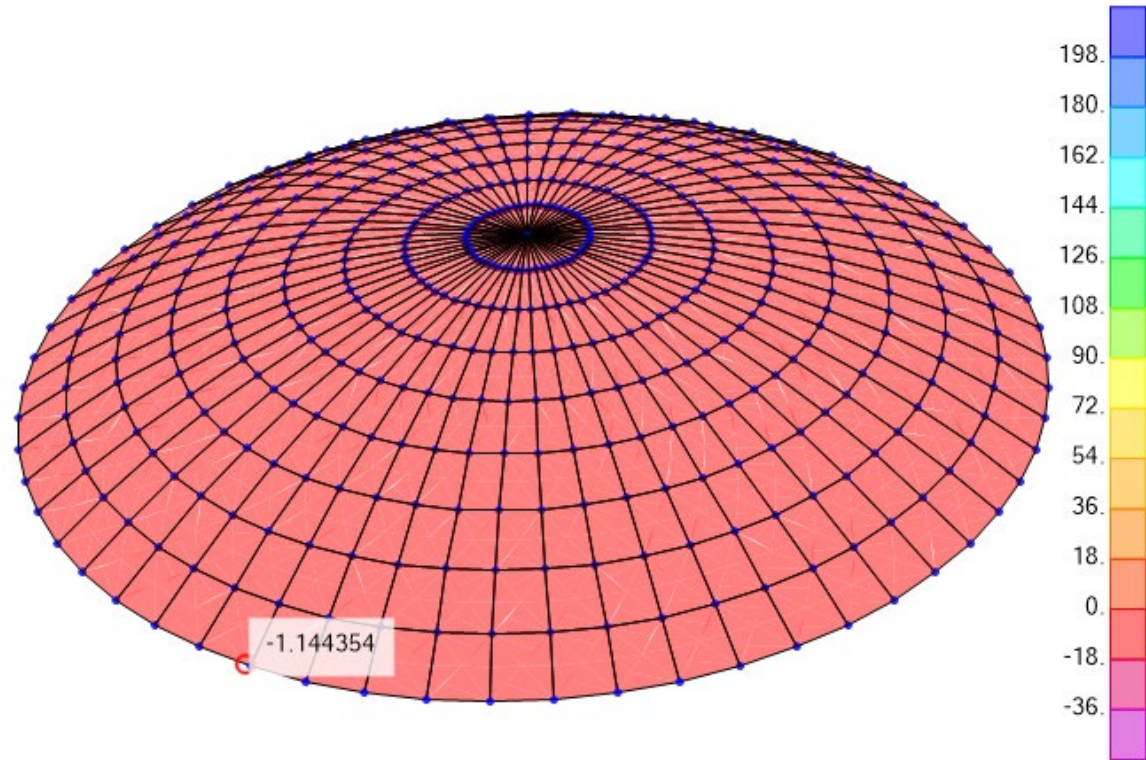

Fuente: Elaboración Propia

Figura 34-III: Fuerzas actuantes F11 Dirección "Y"
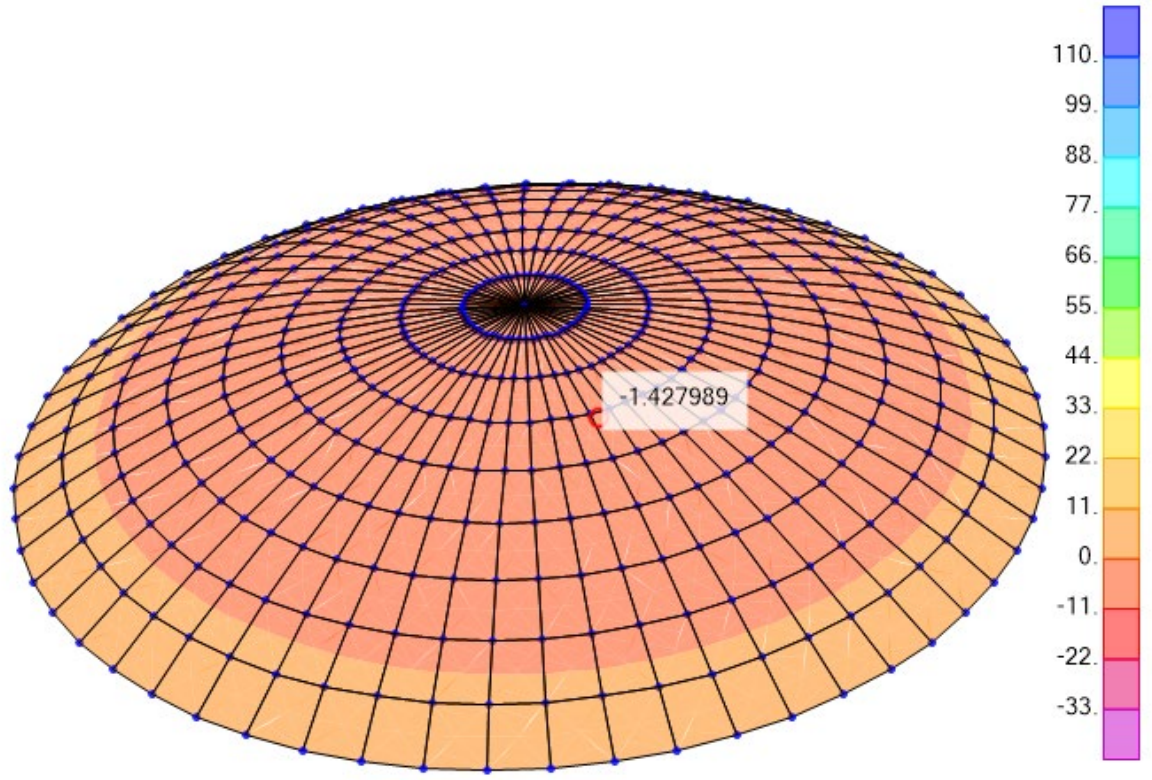

Fuente: Elaboración Propia 
Se analizará de acuerdo a la teoría de membranas Con los esfuerzos que se producen en la cúpula por el peso propio y por los efectos de flexión producidos en el apoyo. En las figuras 36-III y 37-III se muestran los esfuerzos que actúan en la cúpula superior.

\section{DIRECCION DEL MERIDIANO}

$\mathrm{F} 11=4.848 \mathrm{Tn} / \mathrm{m}$

$\mathrm{F} 22=2.792 \mathrm{Tn} / \mathrm{m}$ meridiano

$\mathrm{F}^{\prime} \mathrm{c}=280 \mathrm{~kg} / \mathrm{cm} 2$

$\mathrm{fr}=\quad 33.466 \mathrm{~kg} / \mathrm{cm} 2 \quad=2 \cdot \sqrt{f^{\prime} c}$

$\mathrm{b}=\quad 7.5 \mathrm{~cm}$

$$
\operatorname{Asmin}=\frac{0.7 \cdot \sqrt{f^{\prime} c} \cdot b \cdot d}{F y}
$$

$\mathrm{ad}=100 \quad \mathrm{~cm} \quad$ ancho tributario

$\sigma \mathrm{c}=2.792<33.46640106 \mathrm{~kg} / \mathrm{cm} 2 \quad \mathrm{OK}$

As $\min =\quad 2.092 \mathrm{~cm} 2$

Distribución= $=1 / 2 @ 0.25$

As real $=\quad 2.53 \quad \mathrm{~cm} 2$

\section{DIRECCIÓN HORIZONTAL}

$F^{\prime} c=280 \quad \mathrm{~kg} / \mathrm{cm} 2$

$\mathrm{fr}=\quad 33.466 \mathrm{~kg} / \mathrm{cm} 2 \quad=2\left(\mathrm{f}^{\prime} \mathrm{c}\right){ }^{\wedge} 0.5$

$\mathrm{F} 11=4.848 \mathrm{Tn} / \mathrm{m}$

$\mathrm{b}=\quad 7.5 \mathrm{~cm}$

$\mathrm{ad}=100 \quad \mathrm{~cm} \quad$ ancho tributario 
$\sigma c=4.848<33.466 \mathrm{~kg} / \mathrm{cm} 2 \quad \mathrm{OK}$

As $\min =\quad 2.092 \mathrm{~cm} 2$

Distribución= $11 / 2 @ 0.25$

As real $=\quad 2.53 \quad \mathrm{~cm} 2$

\subsubsection{DISEÑO DE VIGAS}

En la figura 38-III se muestra la ubicación de las vigas a diseñar.

Figura 35-III: Esfuerzos en las Vigas.

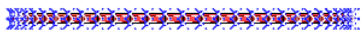

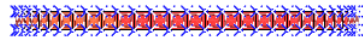

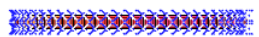
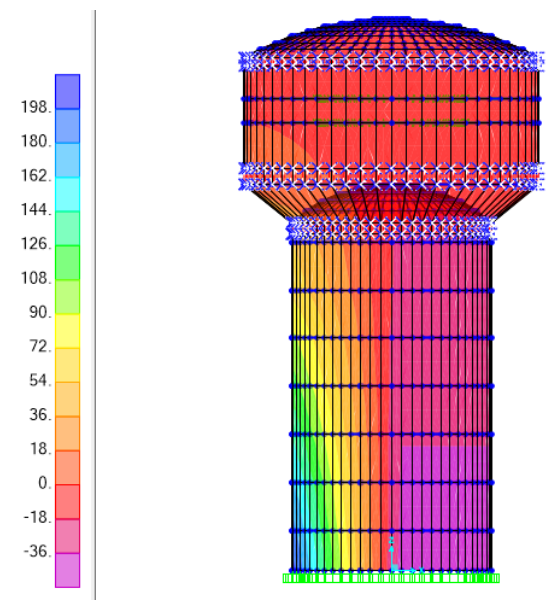

\begin{tabular}{r|r|}
\hline 198. & \\
180. \\
162. \\
144. \\
126. \\
108. \\
90. \\
72. \\
54. \\
36. \\
18. \\
\hline 0. \\
\hline-18. \\
-36. \\
\hline
\end{tabular}

Fuente: Elaboración Propia

\subsubsection{VIGA INFERIOR}

Se muestra en la siguiente tabla las fuerzas que actúan en la viga inferior.

Tabla 24-III: Fuerzas que Actúan en La viga Inferior

\begin{tabular}{|c|r|r|r|}
\hline Combinación & F1 (Tonf) & F2 (Tonf) & F3 (Tonf) \\
\hline ENVOLVENTE & -51.8421 & 62.5278 & 17.151 \\
\hline ENVOLVENTE & -51.7917 & 61.3759 & 15.5852 \\
\hline
\end{tabular}

Fuente: Elaboración propia de resultados obtenidos del programa computacional SAP2000.

Fuerzas para el diseño

$\mathrm{F} 11=51.84 \mathrm{Tnf}$

$\mathrm{F} 22=62.53 \mathrm{Tnf}$ 
Diseño por tracción y verificación de fisuramiento

$\mathrm{b}=0.40 \mathrm{~m}$
$\mathrm{~h}=1.00 \mathrm{~m}$

$\mathrm{f}^{\prime}{ }_{\mathrm{c}}=280 \mathrm{kgf} / \mathrm{cm} 2$

$\mathrm{f}_{\mathrm{y}}=\quad 4200 \mathrm{kgf} / \mathrm{cm} 2$

$\mathrm{f}_{\mathrm{s}}=1400 \mathrm{kgf} / \mathrm{cm} 2 \quad$ Esfuerzo permisible del acero por fisuramiento (ACI 350)

$\mathrm{fs}=0.33$ fy

$\mathrm{Ec}=15000 \cdot \sqrt{f^{\prime} c} \quad \mathrm{~kg} / \mathrm{cm} 2$

$\mathrm{Ec}=280624 \quad \mathrm{kgf} / \mathrm{cm} 2$

Es $=2000000 \quad \mathrm{kgf} / \mathrm{cm} 2 \quad$ Módulo de elasticidad del acero

$\mathrm{n}=\quad \mathrm{Es} / \mathrm{Ec}=7.13$

$\varepsilon s h=0.0003 \quad$ Coeficiente de fisuramiento del concreto armado

$\mathrm{fct}=0.1 \mathrm{f}^{\prime} \mathrm{c} \quad$ Esfuerzo permisible del concreto por fisuramiento

$\mathrm{fct}=35 \mathrm{kgf} / \mathrm{cm} 2$

$\mathrm{T}=\quad 51842.1 \mathrm{kgf} \quad$ Fuerza de tensión del elemento

$\mathrm{t}=\quad 45.69 \mathrm{~cm} \quad$ Espesor del elemento 
Con peralte mayor a t, calculamos el acero por tracción requerido

$\mathrm{As}=\mathrm{Tu} / \varphi f \mathrm{~s}$

$\mathrm{Tu}=\quad 62527.8 \mathrm{kgf}$

$\mathrm{As}=47.64 \mathrm{~cm} 2$

$\mathrm{As}=67.77 \mathrm{~cm} 2$ con una distribución de $10 \varphi 1^{\prime \prime}$

Diseño por Corte

$\mathrm{Av}<\mathrm{S}(\mathrm{Vu}-\varphi \mathrm{Vc}) /\left(\mathrm{f}_{\mathrm{y}} . \mathrm{d}\right) \quad$ Considerando un espaciamiento de $15 \mathrm{~cm}$ se obtiene

$\mathrm{Vu}=62527.8 \mathrm{kgf}$

$\mathrm{Vc}=39662 \mathrm{kgf}$

Av $<1.27 \mathrm{~cm}^{2} \quad$ La cual se toma la barra de $1 / 2 "$

Obteniendo una separación mínima de

$\operatorname{Smin}=12 \mathrm{~cm}$

Por lo tanto, el refuerzo por corte será de:

Sp1/2"@15cm

\subsubsection{VIGA SUPERIOR}

Se muestra en la siguiente tabla las fuerzas que actúan en la viga superior. 
Tabla 25-III: Fuerzas que actúan en la Viga Superior

\begin{tabular}{|c|r|r|r|}
\hline Combinación & F1 (Tonf) & F2 (Tonf) & F3 (Tonf) \\
\hline ENVOLVENTE & -5.0134 & 5.2938 & 0.9187 \\
\hline
\end{tabular}

Fuente: Elaboración propia de resultados obtenidos del programa computacional SAP2000.

Fuerzas de Diseño

$\mathrm{F} 11=5.0134 \mathrm{Tnf}$

$\mathrm{F} 22=5.2938 \mathrm{Tnf}$

Diseño por tracción y verificación de fisuramiento

$$
\begin{array}{lll}
\mathrm{b}= & 0.40 & \mathrm{~m} \\
\mathrm{~h}= & 0.50 \mathrm{~m} \\
\mathrm{f}^{\prime} \mathrm{c}= & 280 & \mathrm{kgf} / \mathrm{cm} 2 \\
\mathrm{fy}= & 4200 \mathrm{kgf} / \mathrm{cm} 2
\end{array}
$$

$\mathrm{fs}=\quad 1400 \mathrm{kgf} / \mathrm{cm} 2 \quad$ Esfuerzo permisible del acero por fisuramiento (ACI 350 - 06)

$\mathrm{fs}=0.33$ fy

$\mathrm{Ec}=15000 \cdot \sqrt{f^{\prime} c} \mathrm{~kg} / \mathrm{cm} 2$

$\mathrm{Ec}=280624 \quad \mathrm{kgf} / \mathrm{cm} 2$

$\mathrm{Es}=2000000 \quad \mathrm{kgf} / \mathrm{cm} 2 \quad$ Módulo de elasticidad del acero

$\mathrm{n}=\mathrm{Es} / \mathrm{Ec}=7.13$

$\varepsilon s h=0.0003 \quad$ Coeficiente de fisuramiento del concreto armado

$\mathrm{fct}=\quad 0.1 \mathrm{f} \mathrm{c} \quad$ Esfuerzo permisible del concreto por fisuramiento

$\mathrm{fct}=35 \mathrm{kgf} / \mathrm{cm} 2$

$\mathrm{T}=\quad 5013.4 \mathrm{kgf} \quad$ Fuerza de tensión del elemento 
$\mathrm{t}=\quad 4.36 \mathrm{~cm} \quad$ Espesor del elemento

Con peralte mayor a t, calculamos el acero por tracción requerido

$\mathrm{As}=\mathrm{Tu} / \varphi \mathrm{fs}$

$\mathrm{Tu}=5293.8 \mathrm{kgf} \quad$ Fuerza cortante última

$\mathrm{As}=4.12 \mathrm{~cm} 2$

$\mathrm{As}=5.07 \mathrm{~cm} 2 \quad 4 \varphi 1 / 2 "$

\section{Diseño por Corte}

$\mathrm{Vu}=5195.9 \mathrm{kgf}$

$\mathrm{Vc}=1983.1 \mathrm{kgf}$

Av $<0.911 \mathrm{~cm} 2 \quad$ La cual se toma la barra de $3 / 8 "$

Obteniendo una separación mínima de

$\operatorname{Smin}=25 \mathrm{~cm}$

Por lo tanto, el refuerzo por corte será de:

Sp3/8"@25 cm

\subsubsection{VIGA DEL FUSTE}

Se muestra en la siguiente tabla las fuerzas que actúan en la viga del fuste.

Tabla 26-III: Fuerzas que actúan en la Viga del Fuste

\begin{tabular}{|c|c|r|r|r|}
\hline Elemento & Combinación & F1 (Tonf) & F2 (Tonf) & F3 (Tonf) \\
\hline Viga Fuste & ENVOLVENTE & -41.9115 & 57.9394 & 26.6177 \\
\hline
\end{tabular}

Fuente: Elaboración propia de resultados obtenidos del programa computacional SAP2000.

Fuerzas de Diseño

$\mathrm{F} 11=41.9115 \quad \mathrm{Tnf}$ 
$\mathrm{F} 22=57.9394 \quad \mathrm{Tnf}$

\section{Diseño por tracción y verificación de fisuramiento}

$$
\begin{array}{lll}
\mathrm{b}= & 0.40 & \mathrm{~m} \\
\mathrm{~h}= & 0.70 & \mathrm{~m} \\
\mathrm{f}^{\prime} \mathrm{c}= & 280 & \mathrm{kgf} / \mathrm{cm} 2 \\
\mathrm{fy}= & 4200 \mathrm{kgf} / \mathrm{cm} 2
\end{array}
$$

$\mathrm{fs}=1400 \mathrm{kgf} / \mathrm{cm} 2 \quad$ Esfuerzo permisible del acero por fisuramiento (ACI $350-06)$

$\mathrm{fs}=0.33$ fy

$\mathrm{Ec}=15000 \cdot \sqrt{f^{\prime} c}$

$\mathrm{Ec}=280624 \quad \mathrm{kgf} / \mathrm{cm} 2$

$\mathrm{Es}=2000000 \quad \mathrm{kgf} / \mathrm{cm} 2 \quad$ Módulo de elasticidad del acero

$\mathrm{n}=\quad \mathrm{Es} / \mathrm{Ec}=7.13$

$\varepsilon s h=0.0003 \quad$ Coeficiente de fisuramiento del concreto armado

$\mathrm{fct}=\quad 0.1 \mathrm{f}^{\prime} \mathrm{c} \mathrm{kgf} / \mathrm{cm} 2 \quad$ Esfuerzo permisible del concreto por fisuramiento

$\mathrm{fct}=35 \quad \mathrm{kgf} / \mathrm{cm} 2$

$\mathrm{T}=\quad 41911.5 \quad \mathrm{kgf} \quad$ Fuerza de tensión del elemento

$\mathrm{t}=\quad 39.09 \mathrm{~cm} \quad$ Espesor del elemento

Con peralte mayor a t, calculamos el acero por tracción requerido 


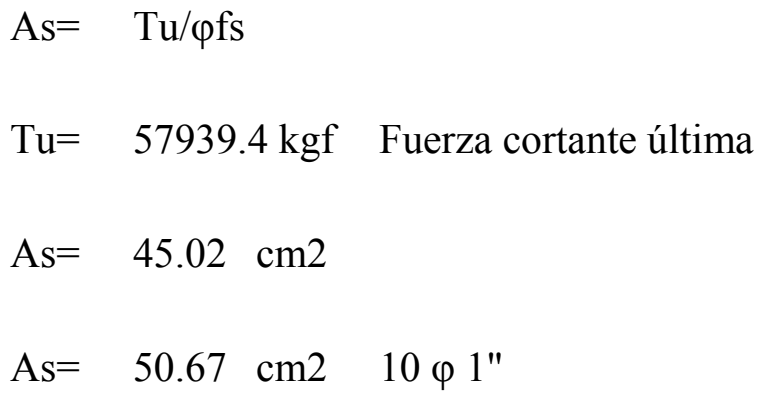

\section{Diseño por Corte}

$\mathrm{Av}<\mathrm{S}(\mathrm{Vu}-\varphi \mathrm{Vc}) /(\mathrm{fy} . \mathrm{d}) \quad$ Considerando un espaciamiento de $15 \mathrm{~cm}$ se obtiene

$\mathrm{Vu}=56727 \mathrm{kgf}$

$\mathrm{Vc}=27763 \mathrm{kgf}$

Av $<1.38 \mathrm{~cm} 2 \quad$ La cual se toma la barra de $1 / 2 "$

Obteniendo una separación mínima de

$\operatorname{Smin}=12 \mathrm{~cm}$

Por lo tanto, el refuerzo por corte será de:

Sp1/2"@15 cm

\subsubsection{DISEÑO DE CUBA}

Para el diseño de la cuba se tendrá en cuenta el diseño por agrietamiento. En la figura 39-III se muestra la vista de la cuba.

$$
A s=\frac{M}{f s . j . d} \quad f s=0,6 . f y \quad j=1-\frac{k}{3} \quad k=\frac{1}{1+\frac{f s}{f c}}
$$


Figura 36-III: Esfuerzos que actúan en la Cuba

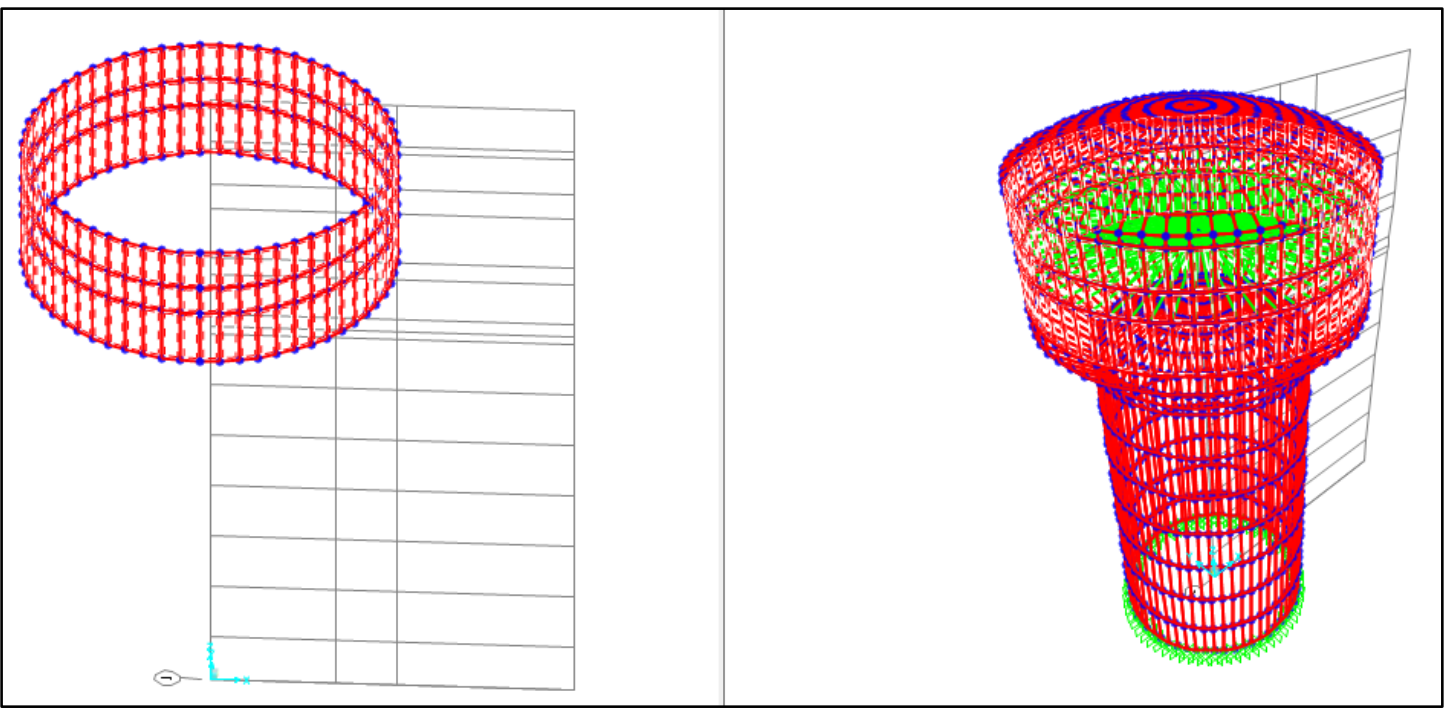

Fuente: Elaboración Propia

En la siguiente tabla se muestran las fuerzas y momentos que actúan sobre la cuba.

Tabla 27-III: Fuerzas y momentos que actúan sobre el elemento Cuba

\begin{tabular}{|c|c|c|c|c|}
\hline Combinación & $\begin{array}{c}\text { F1 } \\
\text { (Tonf) }\end{array}$ & $\begin{array}{c}\text { F2 } \\
\text { (Tonf) }\end{array}$ & $\begin{array}{c}\text { M1 } \\
\text { (Tonf-m) }\end{array}$ & $\begin{array}{c}\text { M2 } \\
\text { (Tonf-m) }\end{array}$ \\
\hline ENVOLVENTE & 62.669 & 106.067 & 13.816 & 8.823 \\
\hline
\end{tabular}

Fuente: Elaboración propia de resultados obtenidos del programa computacional SAP2000.

Fuerzas y Momentos para el diseño:

$\mathrm{F} 11=62.669 \mathrm{Tnf}$

$\mathrm{F} 22=106.067 \mathrm{Tnf}$

$\mathrm{M} 11=13.816$ Tnf.m

$\mathrm{M} 22=8.823$ Tnf.m

\section{Diseño por tracción y verificación de fisuramiento}

$\mathrm{b}=1.00 \mathrm{~m}$ 


$$
\begin{array}{lll}
f^{\prime} c= & 350 & \mathrm{kgf} / \mathrm{cm} 2 \\
\mathrm{fy}= & 4200 & \mathrm{kgf} / \mathrm{cm} 2
\end{array}
$$

$\mathrm{fs}=1400 \mathrm{kgf} / \mathrm{cm} 2 \quad$ Esfuerzo permisible del acero por fisuramiento (ACI $350-06)$

fs $=0.33$ fy

$\mathrm{Ec}=15000 \cdot \sqrt{f^{\prime} c} \quad \mathrm{~kg} / \mathrm{cm} 2$

$\mathrm{Ec}=280624 \mathrm{kgf} / \mathrm{cm} 2$

Es $=2000000 \mathrm{kgf} / \mathrm{cm} 2 \quad$ Módulo de elasticidad del acero

$\mathrm{n}=\quad \mathrm{Es} / \mathrm{Ec}=7.13$

$\varepsilon \mathrm{sh}=0.0003$

Coeficiente de fisuramiento del concreto armado

$\mathrm{fct}=0.1 \mathrm{f}^{\prime} \mathrm{c}$

Esfuerzo permisible del concreto por fisuramiento

$\mathrm{fct}=\quad 35 \mathrm{kgf} / \mathrm{cm} 2$

$\mathrm{T}=\quad 62669 \mathrm{kgf} \quad$ Fuerza de tensión del elemento

$\mathrm{t}=22.39 \mathrm{~cm} \quad$ Espesor del elemento

Cálculo del acero por tracción
$\mathrm{As}=\mathrm{Tu} / \varphi \mathrm{fs}$
$\mathrm{Tu}=62669 \mathrm{kgf}$
Fuerza cortante última 
$\mathrm{As}=49.74 \mathrm{~cm} 2$

As $($ diseño $)=50.70 \mathrm{~cm} 2$

Espaciamiento en 2 capas de 1"

$\mathrm{S}(1 ")=\quad 20.38 \mathrm{~cm}$

Entonces:

S(1”)@20cm

\section{Diseño por Flexión}

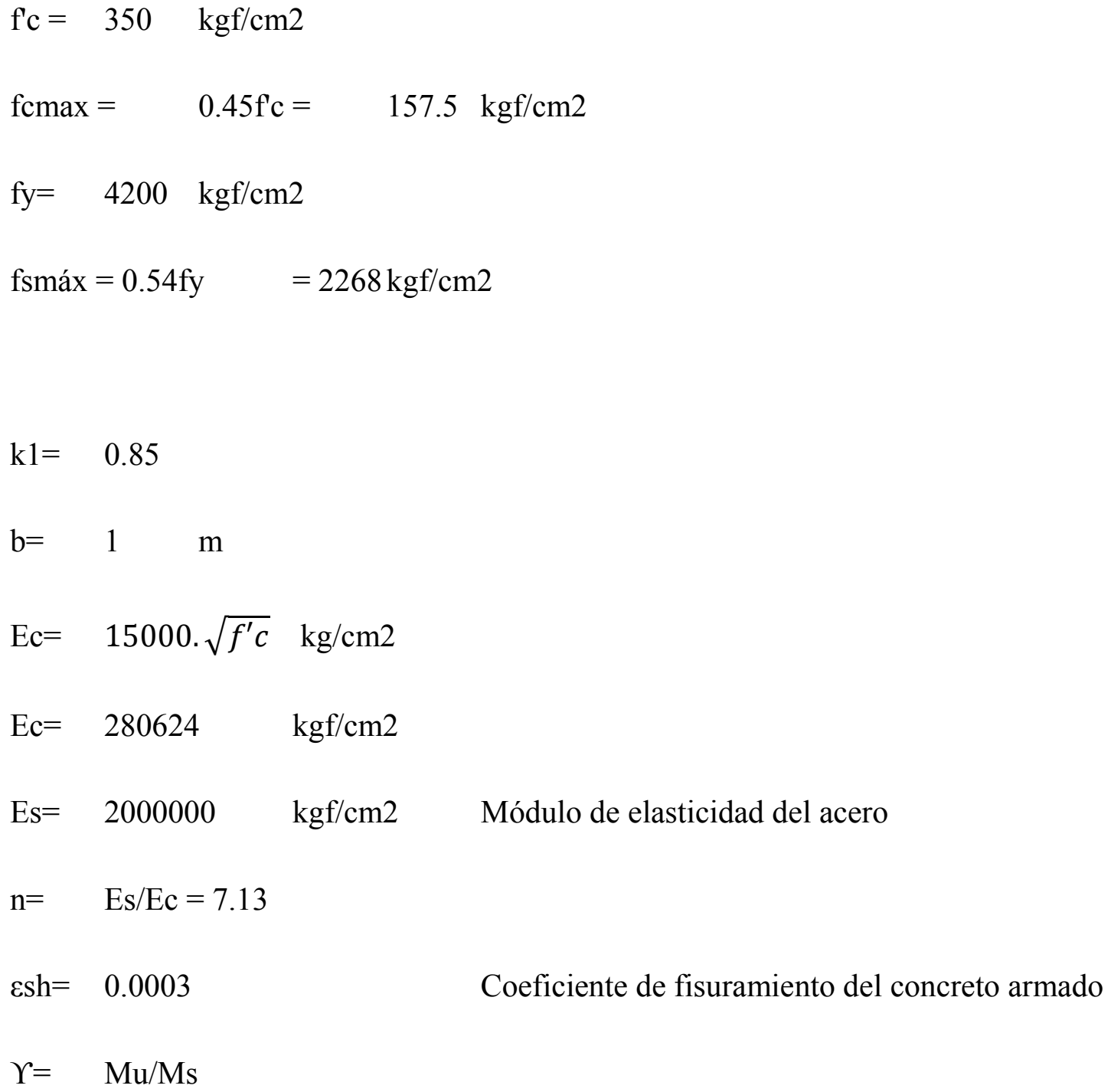


$\Upsilon=1.4$

$\varphi=0.9$

Factor de Durabilidad

$$
S d=\max \left(\varphi \cdot \frac{f y}{y} \cdot f s_{\max } ; 1\right)
$$

Cuantía de Refuerzo

$\mathrm{Sd}=$ 1.190

$\rho \mathrm{b}=\frac{0,85 \cdot k 1 \cdot f^{\prime} c}{f y} \cdot\left(\frac{M_{11}}{M_{11}+f_{y}}\right)$

$\rho b=0.0627$

$\rho \max =\frac{f^{\prime} c_{\max }}{2 \cdot f s_{\max }} \cdot \frac{1}{\left(1+\frac{f s_{\max }}{n \cdot f^{\prime} c_{\max }}\right)}$

$\rho \max =\quad 0.0115$

$\rho \max / \rho b=0.183$

$\operatorname{Kumax}=\rho_{\max } \cdot f y \cdot\left(1-\left(\frac{0,5 \cdot \rho_{\max } \cdot f y}{0,85 \cdot f^{\prime} c}\right)\right.$

Kumax $=\quad 44.363 \mathrm{kgf} / \mathrm{cm} 2$ 
dmín $=\sqrt{\frac{S d \cdot M u}{\varphi * K u_{\max } \cdot b}}$

dmín= $19.69 \mathrm{~cm}$

Espesor Mínimo

$\mathrm{hmin}=\mathrm{dmin}+\mathrm{Cc}+1.00 \mathrm{~cm}$

$\mathrm{hmin}=25.69 \mathrm{~cm}$

El espesor por tracción requerido en el punto anterior es de $\mathrm{h}=30 \mathrm{~cm}$

$\mathrm{d}=\mathrm{h}-\mathrm{Cc}-1.0 \mathrm{~cm}$

$\mathrm{d}=24 \quad \mathrm{~cm}$

$\mathrm{Ku}=\frac{S d \cdot M u}{\varphi \cdot b \cdot d^{2}}$

$\mathrm{Ku}=29.854 \mathrm{kgf} / \mathrm{cm} 2$

$\rho=\frac{0,85 \cdot f^{\prime} c}{f y} \cdot\left(\frac{1-\sqrt{1-4 \cdot 0,59 \cdot K u}}{2 \cdot 0,59}\right)$

$\rho=0.00751$

$\mathrm{As}=\rho . b . \mathrm{d}$

$\mathrm{As}=18.01 \mathrm{~cm} 2$

Acero Mínimo

Asmin $=\operatorname{Max}\left(\sqrt[3]{f^{\prime} c .1 p s i} \cdot b \cdot \frac{d}{f y} ; 200 \cdot \frac{(1 p s i)(b . d)}{f y}\right)$ 
$\mathrm{As} 1=1.65965307 \quad \mathrm{~cm} 2$

$\mathrm{As} 2=8.00 \quad \mathrm{~cm} 2$

Asmin $=\quad 8.00 \quad \mathrm{~cm} 2$

Espaciamiento:

$\mathrm{S}\left(\varphi 3 / 4^{\prime \prime}\right)=15.82 \mathrm{~cm}$

Diseño por Tracción y verificación por fisuramiento

$\mathrm{As}=\mathrm{Tu} / \varphi \mathrm{fs}$

$\mathrm{As}=84.18 \mathrm{~cm} 2$

Espaciamiento en 2 capas de 1"

$\mathrm{S}\left(\varphi 1^{\prime \prime}\right)=12.04 \mathrm{~cm}$

Por tanto: (Sp1")@10 cm

Espaciamiento mínimo por fisuramiento:

$$
\begin{gathered}
S(\mathrm{~cm}) \leq 38\left(\frac{2800}{f s}\right)-2,5 . r c \leq 20.5 \mathrm{~cm}(O K) \\
S(\mathrm{~cm}) \leq 30\left(\frac{2800}{f s}\right) \leq 30 \mathrm{~cm}(O K)
\end{gathered}
$$

\subsubsection{DISEÑO DEL FUSTE}

En la siguiente figura se muestra la vista del elemento estructural Fuste. 
Figura 37-III: Diseño de Fuste

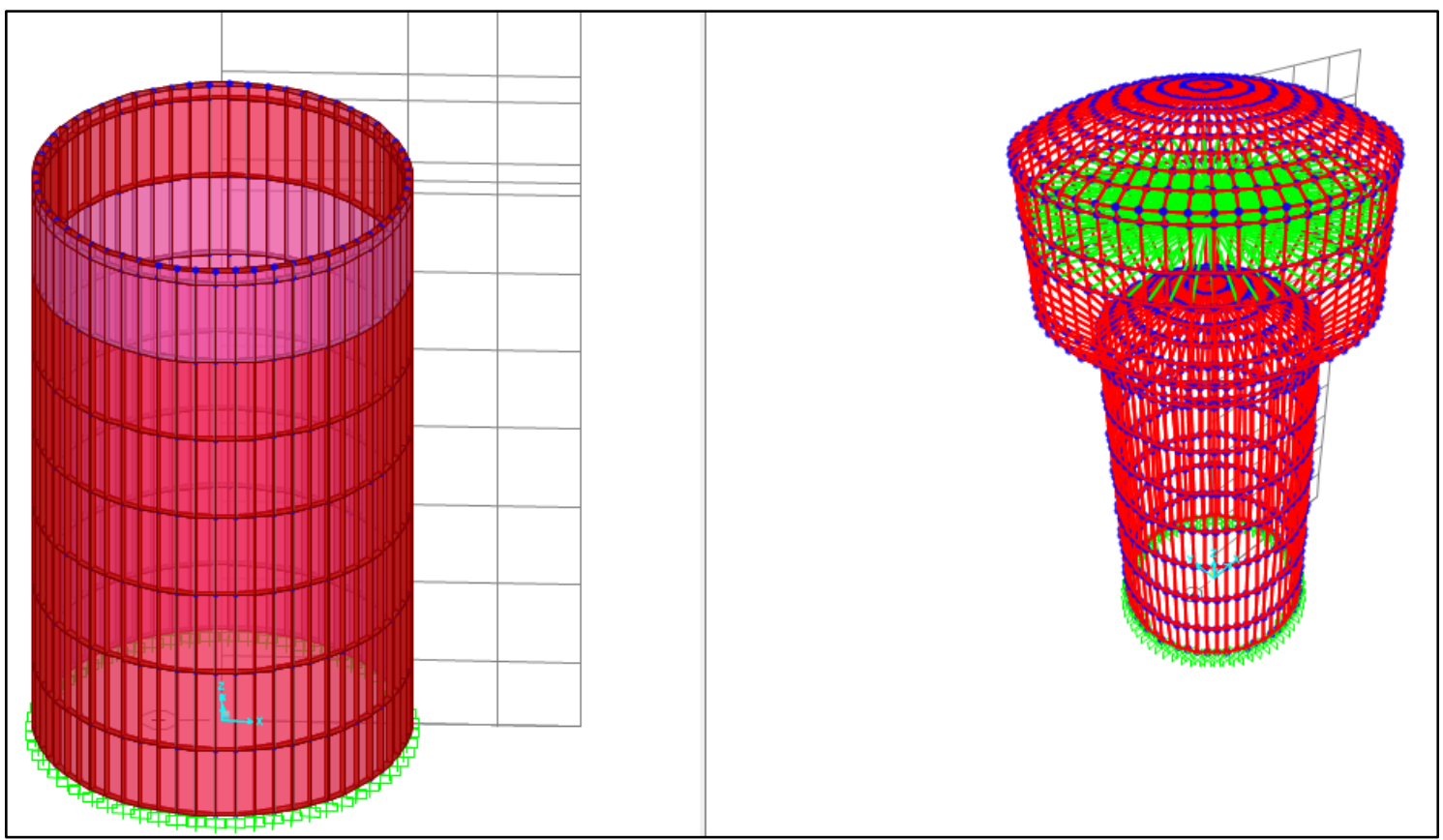

\begin{tabular}{|c|c|c|c|c|}
\hline $\begin{array}{c}\text { Fuente: } \\
\text { Elaboración } \\
\text { Propia } \\
\text { Combinación }\end{array}$ & F1 & F2 & M1 & M2 \\
(Tonf) & (Tonf) & (Tonf-m) & (Tonf-m) \\
\hline ENVOLVENTE & 33.968 & 49.481 & 6.241 & 4.137 \\
\hline
\end{tabular}

Fuerzas y Momentos para el diseño:

$\mathrm{F} 11=33.968 \mathrm{Tnf}$

$\mathrm{F} 22=49.481 \mathrm{Tnf}$

M11= 6.241 Tnf.m

$\mathrm{M} 22=4.137$ Tnf.m

Diseño por tracción y verificación de fisuramiento

$\mathrm{b}=1.00 \mathrm{~m}$ 


$$
\begin{array}{lll}
f^{\prime} c= & 350 & \mathrm{kgf} / \mathrm{cm} 2 \\
\mathrm{fy}= & 4200 & \mathrm{kgf} / \mathrm{cm} 2
\end{array}
$$

$\mathrm{fs}=1400 \mathrm{kgf} / \mathrm{cm} 2 \quad$ Esfuerzo permisible del acero por fisuramiento (ACI $350-06)$

fs $=0.33$ fy

$\mathrm{Ec}=15000 \cdot \sqrt{f^{\prime} c} \quad \mathrm{~kg} / \mathrm{cm} 2$

$\mathrm{Ec}=280624 \mathrm{kgf} / \mathrm{cm} 2$

Es $=2000000 \mathrm{kgf} / \mathrm{cm} 2 \quad$ Módulo de elasticidad del acero

$\mathrm{n}=\quad \mathrm{Es} / \mathrm{Ec}=7.13$

$\varepsilon s h=0.0003$

Coeficiente de fisuramiento del concreto armado

$\mathrm{fct}=0.1 \mathrm{f}^{\prime} \mathrm{c}$

Esfuerzo permisible del concreto por fisuramiento

$\mathrm{fct}=\quad 35 \mathrm{kgf} / \mathrm{cm} 2$

$\mathrm{T}=62669 \mathrm{kgf} \quad$ Fuerza de tensión del elemento

$\mathrm{t}=\quad 12.14 \mathrm{~cm} \quad$ Espesor del elemento

Cálculo del acero por tracción
$\mathrm{As}=\mathrm{Tu} / \varphi \mathrm{fs}$
$\mathrm{Tu}=33968 \mathrm{kgf}$
Fuerza cortante última
$\mathrm{As}=26.96 \mathrm{~cm} 2$ 
As $($ diseño $)=28.50 \mathrm{~cm} 2$

Espaciamiento en 2 capas de 3/4"

$\mathrm{S}\left(3 / 4^{\prime \prime}\right)=20.38 \mathrm{~cm}$

Entonces:

S(3/4”)@20cm

\section{Diseño por Flexión}

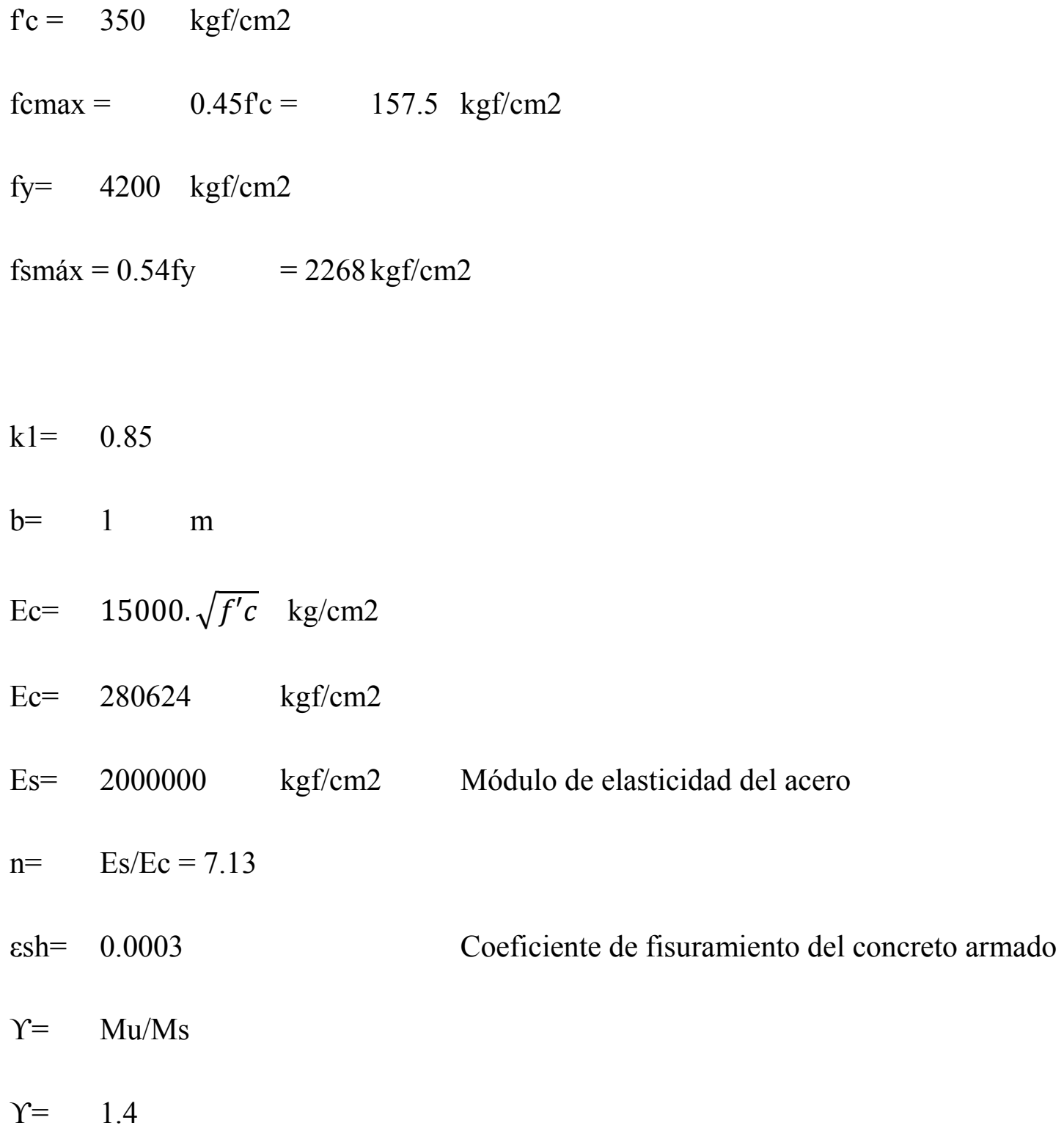


$\varphi=0.9$

Factor de Durabilidad

$$
S d=\max \left(\varphi \cdot \frac{f y}{y} \cdot f s_{\max } ; 1\right)
$$

$$
\mathrm{Sd}=\quad 1.190
$$

Cuantía de Refuerzo

$$
\begin{aligned}
\rho b & =\frac{0,85 \cdot k 1 \cdot f^{\prime} c}{f y} \cdot\left(\frac{M_{11}}{M_{11}+f_{y}}\right) \\
\rho b & =0.0627
\end{aligned}
$$

$\rho \max =\frac{f^{\prime} c_{\max }}{2 \cdot f s_{\max }} \cdot \frac{1}{\left(1+\frac{f s_{\max }}{n \cdot f^{\prime} c_{\max }}\right)}$

$\rho \max =\quad 0.0115$

$\rho \max / \rho b=0.183$

$\operatorname{Kumax}=\rho_{\max } \cdot f y \cdot\left(1-\left(\frac{0,5 \cdot \rho_{\max } \cdot f y}{0,85 \cdot f^{\prime} c}\right)\right.$

Kumax $=\quad 44.363 \mathrm{kgf} / \mathrm{cm} 2$

dmín $=\sqrt{\frac{S d \cdot M u}{\varphi * K u_{\max } \cdot b}}$ 
dmín= $19.69 \mathrm{~cm}$

Espesor Mínimo

$\mathrm{hmin}=\mathrm{dmin}+\mathrm{Cc}+1.00 \mathrm{~cm}$

$\mathrm{hmin}=25.69 \mathrm{~cm}$

El espesor por tracción requerido en el punto anterior es de $\mathrm{h}=25 \mathrm{~cm}$

$\mathrm{d}=\mathrm{h}-\mathrm{Cc}-1.0 \mathrm{~cm}$

$\mathrm{d}=\quad 19 \quad \mathrm{~cm}$

$\mathrm{Ku}=\frac{S d \cdot M u}{\varphi \cdot \mathrm{b} \cdot d^{2}}$

$\mathrm{Ku}=\quad 47.634 \mathrm{kgf} / \mathrm{cm} 2$

$\rho=\frac{0,85 \cdot f^{\prime} c}{f y} \cdot\left(\frac{1-\sqrt{1-4 \cdot 0,59 \cdot K u}}{2 \cdot 0,59}\right)$

$\rho=0.01243$

$A s=\rho \cdot b \cdot d$

$\mathrm{As}=23.62 \mathrm{~cm} 2$

Acero Mínimo

Asmin $=\operatorname{Max}\left(\sqrt[3]{f^{\prime} c .1 p s i} \cdot b \cdot \frac{d}{f y} ; 200 \cdot \frac{(1 p s i)(b . d)}{f y}\right)$ 
$\mathrm{As} 1=1.313892011 \mathrm{~cm} 2$

$\mathrm{As} 2=6.33 \mathrm{~cm} 2$

Asmin $=\quad 6.33 \mathrm{~cm} 2$

Espaciamiento:

$\mathrm{S}(\varphi 3 / 4 ")=12.07 \mathrm{~cm}$

Diseño por Tracción y verificación por fisuramiento

$\mathrm{As}=\mathrm{Tu} / \varphi \mathrm{fs}$

$\mathrm{As}=39.27 \mathrm{~cm} 2$

Espaciamiento en 2 capas de 1"

$\mathrm{S}(\varphi 3 / 4 ")=25.81 \mathrm{~cm}$

Por tanto: (S甲3/4”)@25 cm

\subsubsection{DISEÑO DE FONDO TRONCOCÓNICO}

En la figura 41-III se muestra la vista del fondo tronco cónico en SAP2000.

Figura 38-III: Esfuerzos en Fondo Troncocónico 


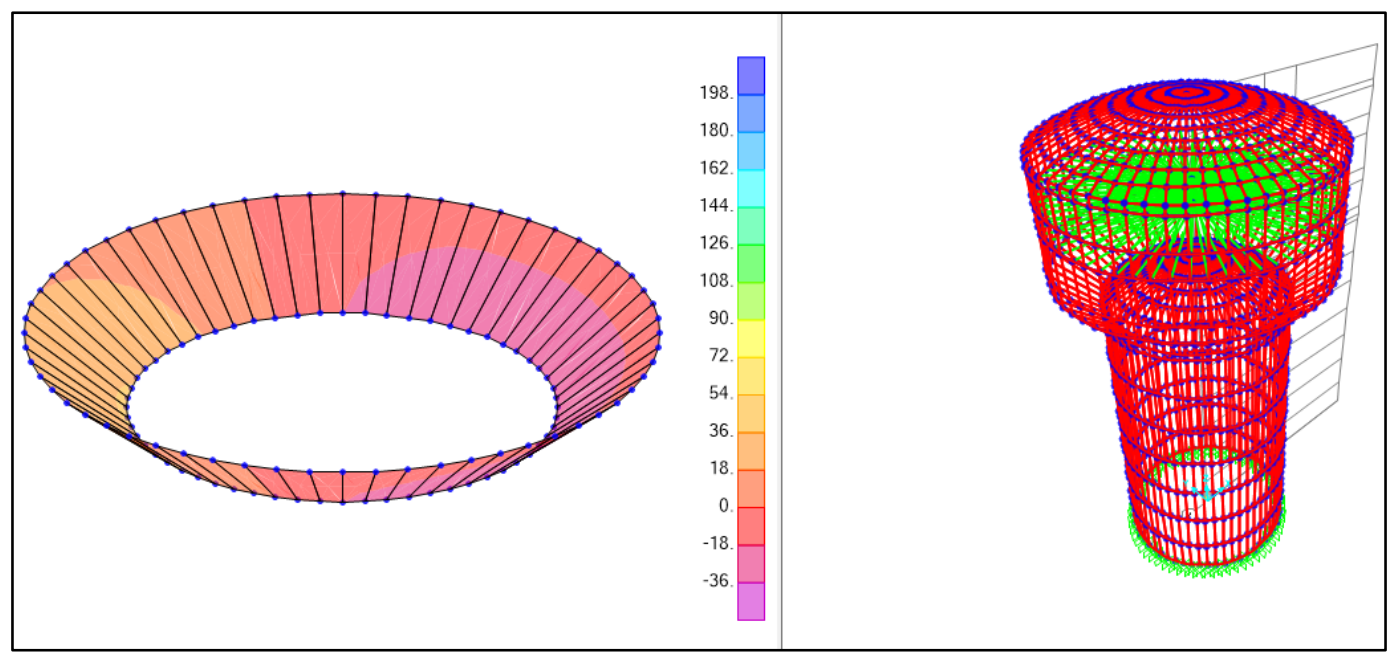

Fuente: Elaboración Propia

En la siguiente tabla se muestran las fuerzas y momentos que actúan sobre el fondo tronco cónico.

Tabla 28: Fuerzas actuantes en el Fondo Troncocónico

\begin{tabular}{|c|c|c|c|c|c|}
\hline Combinación & F1 & F2 & M1 & M2 & M3 \\
\hline & Tonf & Tonf & Tonf-m & Tonf-m & Tonf-m \\
\hline ENVOLVENTE & 912.754 & 1.084 .385 & 6.247 .798 & -3.828 .906 & 1.065 .639 \\
\hline
\end{tabular}

Fuente: Elaboración propia de resultados obtenidos del programa computacional SAP2000.

$\mathrm{F} 11=91.2754 \quad \mathrm{Tnf}$

$\mathrm{F} 22=108.4385$ Tnf

$\mathrm{M} 11=62.47798 \quad$ Tnf.m

$\mathrm{M} 22=38.28906$ Tnf.m

$\mathrm{V} 13=2.203 \mathrm{Tnf}$

$\mathrm{V} 23=5.545 \mathrm{Tnf}$

Diseño por tracción y verificación de fisuramiento

$\mathrm{b}=1.00 \mathrm{~m}$ 


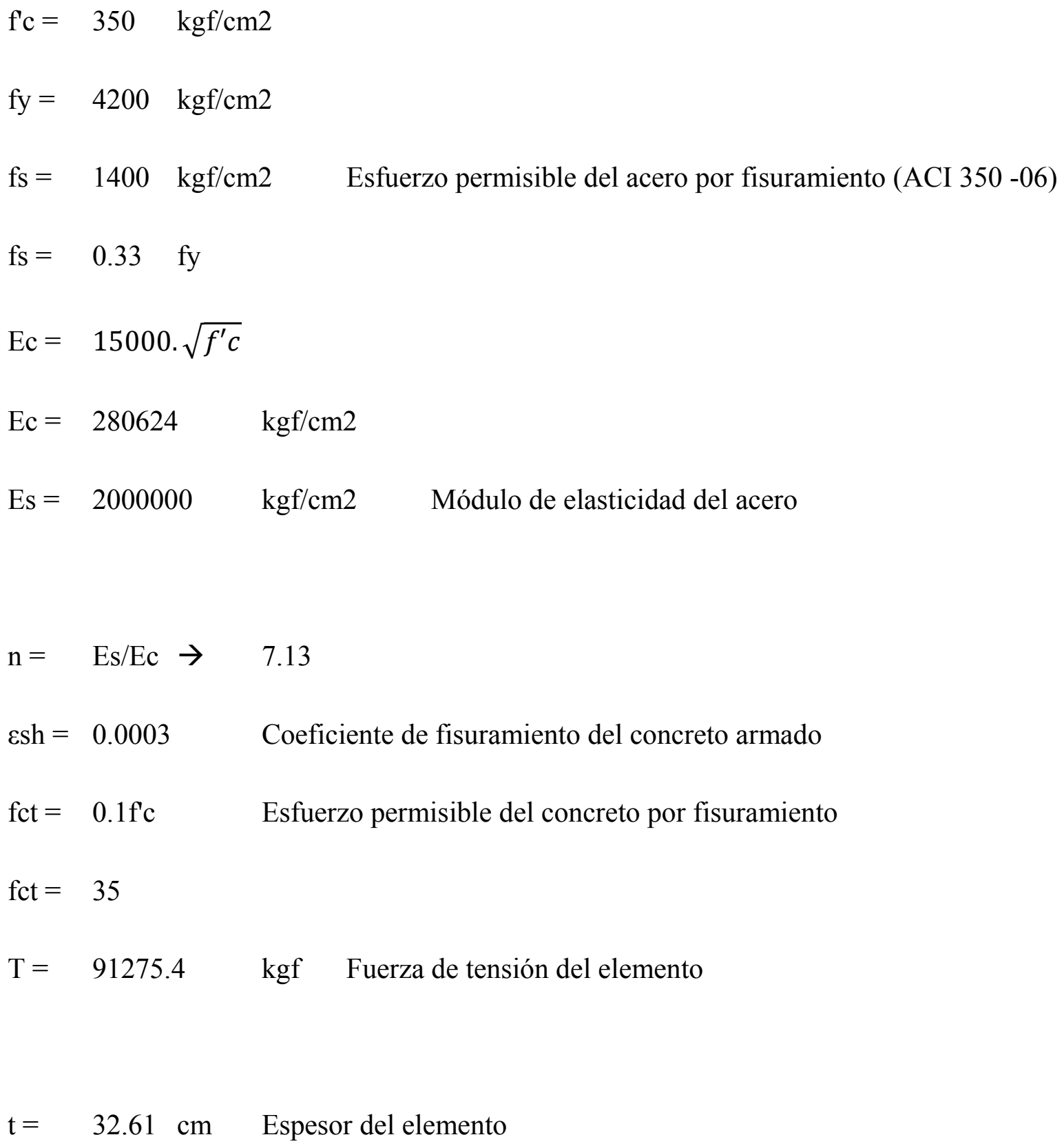


Espaciamiento en 2 capas de 3/4"

$\mathrm{S}\left(3 / 4^{\prime \prime}\right)=7.87 \quad \mathrm{~cm}$

Entonces:

S(3/4")@7.5 cm

Diseño por Flexión

$\mathrm{f}^{\prime} \mathrm{c}=350 \quad \mathrm{kgf} / \mathrm{cm} 2$

$f c \max =\quad 0.45 f^{\prime} \mathrm{c} 157.5 \mathrm{kgf} / \mathrm{cm} 2$

$f y=4200 \quad \mathrm{kgf} / \mathrm{cm} 2$

fsmáx $=\quad 0.54$ fy $2268 \mathrm{kgf} / \mathrm{cm} 2$

$\mathrm{k} 1=0.85$

$b=1 \quad m$

$\mathrm{Ec}=15000 \cdot \sqrt{f^{\prime} c}$

$\mathrm{Ec}=280624 \quad \mathrm{kgf} / \mathrm{cm} 2$

$\mathrm{Es}=2000000 \quad \mathrm{kgf} / \mathrm{cm} 2 \quad$ Módulo de elasticidad del acero

$\mathrm{n}=\quad$ Es/Ec --- $\quad 7.13$

$\varepsilon s h=0.0003 \quad$ Coeficiente de fisuramiento del concreto armado

$\Upsilon=\mathrm{Mu} / \mathrm{Ms}$

$\Upsilon=1.4$ 
$\varphi=0.9$

\section{Factor de Durabilidad}

$\mathrm{Sd}=\operatorname{Max}\left(\varphi \cdot \frac{\mathrm{fy}}{\mathrm{y}} \cdot f s_{\max } ; 1\right)$

$\mathrm{Sd}=1.190$

\section{Cuantía de Refuerzo}

$$
\begin{aligned}
& \rho \mathrm{b}=\frac{0,85 \cdot k 1 \cdot f^{\prime} c}{f y} \cdot\left(\frac{M_{11}}{M_{11}+f_{y}}\right) \\
& \rho b=0.0324
\end{aligned}
$$

$\rho \max =\frac{f \prime c_{\max }}{2 \cdot f s_{\max }} \cdot \frac{1}{\left(1+\frac{f s_{\max }}{n \cdot f^{\prime} c_{\max }}\right)}$

$\rho \max =0.0115$

$\rho \max / \rho b=0.354$

$\operatorname{Kumax}=\rho_{\text {max }} \cdot f y \cdot\left(1-\left(\frac{0,5 \cdot \rho_{\max } \cdot f y}{0,85 \cdot f^{\prime} c}\right)\right.$

Kumax $=44.363 \quad \mathrm{kgf} / \mathrm{cm} 2$ 
dmín $=\sqrt{\frac{S d \cdot M u}{\varphi * K u_{\max } \cdot b}}$

dmín $=10.02 \mathrm{~cm}$

\section{Espesor Mínimo}

$\mathrm{hmin}=\mathrm{dmin}+\mathrm{Cc}+1.00 \mathrm{~cm}$

$\mathrm{hmin}=16.02 \mathrm{~cm}$

El espesor por tracción requerido en el punto anterior es de $\mathrm{h}=30 \mathrm{~cm}$

$\mathrm{d}=\mathrm{h}-\mathrm{Cc}-1.0 \mathrm{~cm}$

$\mathrm{d}=\quad 24 \quad \mathrm{~cm}$

$\mathrm{Ku}=\frac{S d \cdot M u}{\varphi \cdot \mathrm{b} \cdot d^{2}}$

$\mathrm{Ku}=7.734 \mathrm{kgf} / \mathrm{cm} 2$

$\rho=\frac{0,85 \cdot f^{\prime} c}{f y} \cdot\left(\frac{1-\sqrt{1-4 \cdot 0,59 \cdot K u}}{2 \cdot 0,59}\right)$

$\rho=0.00187$

$\mathrm{As}=$ p.b.d

$\mathrm{As}=4.48 \quad \mathrm{~cm} 2$

\section{Acero Mínimo}




$$
\begin{aligned}
& \text { Asmin }=\operatorname{Max}\left(\sqrt[3]{f^{\prime} c \cdot 1 p s i} \cdot b \cdot \frac{d}{f y} ; 200 \cdot \frac{(1 p s i)(b . d)}{f y}\right) \\
& \text { As } 1=1.66 \quad \mathrm{~cm} 2 \\
& \text { As2 }=8.00 \mathrm{~cm} 2
\end{aligned}
$$

Asmin $=\quad 8.00 \quad \mathrm{~cm} 2$

\section{Espaciamiento:}

$\mathrm{S}\left(\varphi 3 / 4^{\prime \prime}\right)=28.28 \mathrm{~cm}$

\section{Diseño por Tracción y verificación por fisuramiento}

$\mathrm{As}=\mathrm{Tu} / \varphi \mathrm{fs}$

$\mathrm{As}=86.06 \mathrm{~cm} 2$

Espaciamiento en 2 capas de 1"

$\mathrm{S}\left(\varphi 1^{\prime \prime}\right)=\quad 11.78 \mathrm{~cm}$

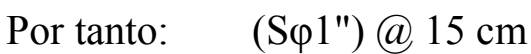

\subsubsection{DISEÑO DE CÚPULA INFERIOR}

En la siguiente tabla se muestran las fuerzas que actúan sobre la cúpula inferior.

Tabla 29-III: Fuerzas que Actúan en la Cúpula Inferior

\begin{tabular}{|c|c|c|r|r|c|}
\hline S22Top & \multicolumn{1}{|c|}{ S22Top } & M11 & M22 & \multicolumn{1}{c|}{ F11 } & \multicolumn{1}{c|}{ F22 } \\
\hline Tonf/m2 & Tonf/m2 & Tonf-m/m & Tonf- $\mathrm{m} / \mathrm{m}$ & Tonf $/ \mathrm{m}$ & Tonf $/ \mathrm{m}$ \\
\hline-359.11 & -238.94 & 256.697 & 321.247 & -91.032 & -62.669 \\
\hline
\end{tabular}

Fuente: Elaboración propia de resultados obtenidos del programa computacional SAP2000. 
Diseño de losa de fondo en la cúpula esférica

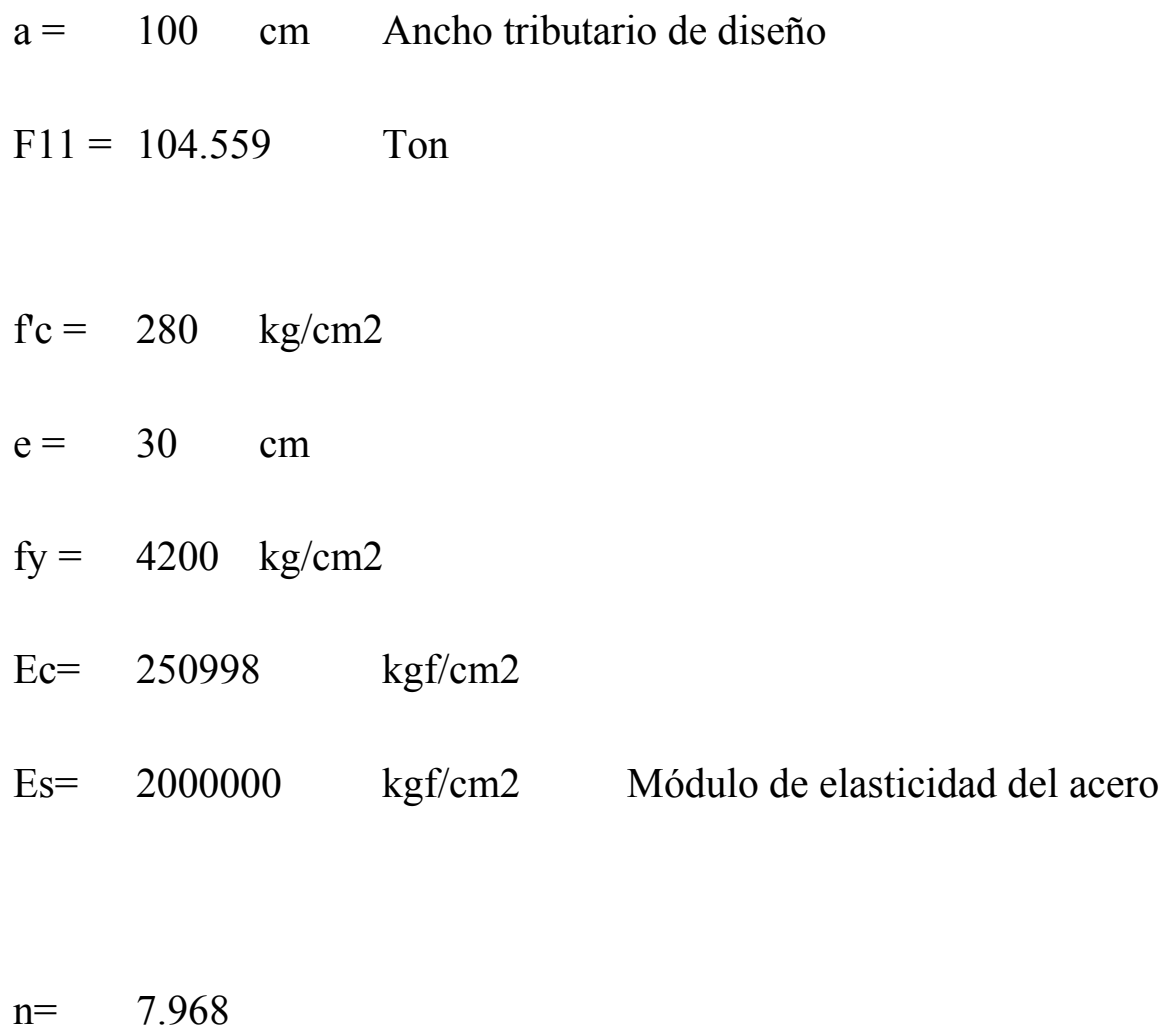

Se verifica que el esfuerzo de compresión no exceda el esfuerzo de concreto:

$\sigma c=2 \cdot \sqrt{f^{\prime} c}$

$\mathrm{f}^{\prime} \mathrm{c}=350 \mathrm{~kg} / \mathrm{cm} 2$

$\sigma c=37.417 \mathrm{kgf} / \mathrm{cm} 2$

$\sigma \mathrm{S} 12=32.659 \mathrm{kgf} / \mathrm{cm} 2$

$\sigma c=37.417 \mathrm{kgf} / \mathrm{cm} 2 \quad>\quad \sigma \mathrm{S} 12=32.659 \mathrm{kgf} / \mathrm{cm} 2 \quad$ Cumple

\section{Diseño de Armadura meridional}

$\mathrm{F} 22=91.032 \mathrm{tnf}$

$\mathrm{b}=1.00 \mathrm{~m}$ 


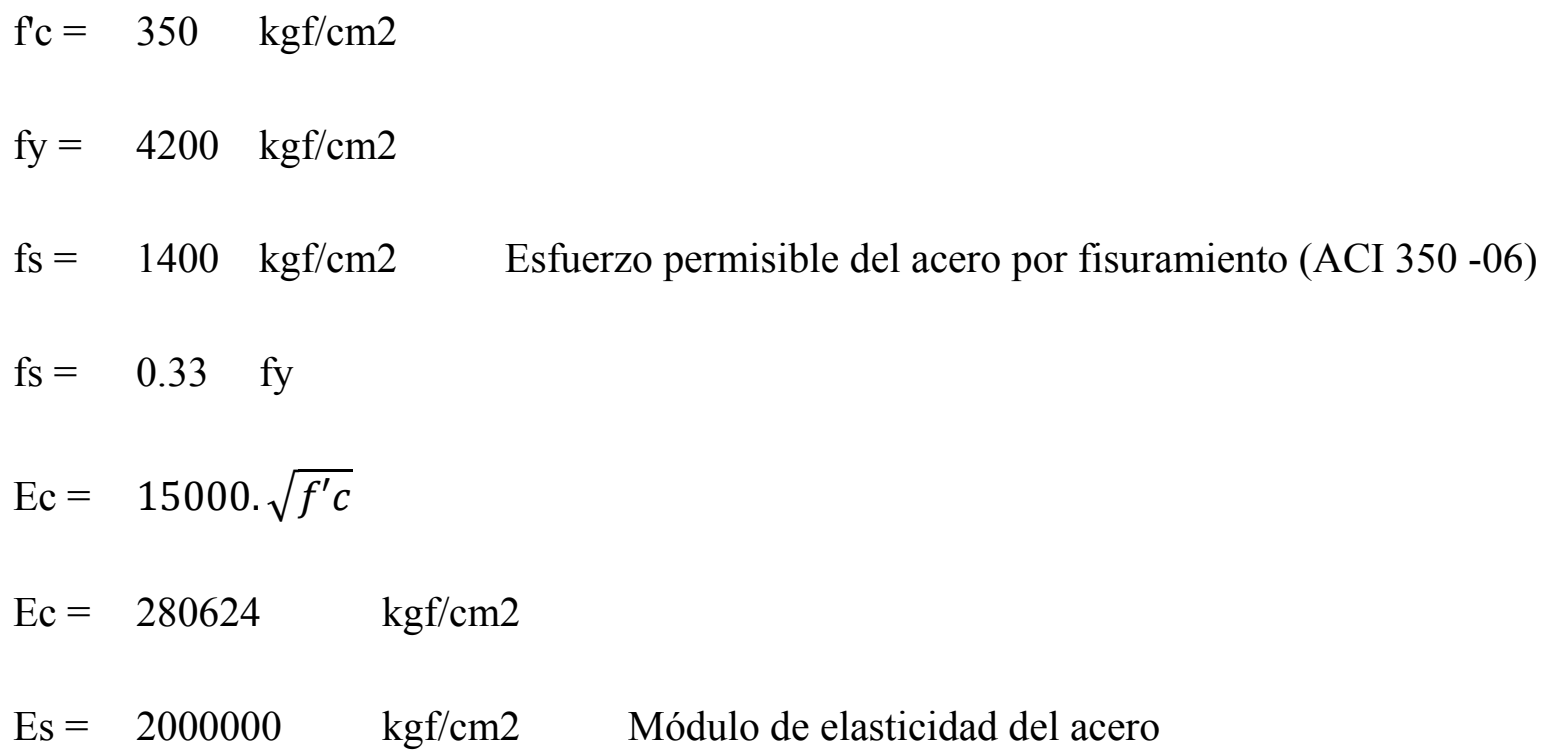

Cálculo del acero por tracción

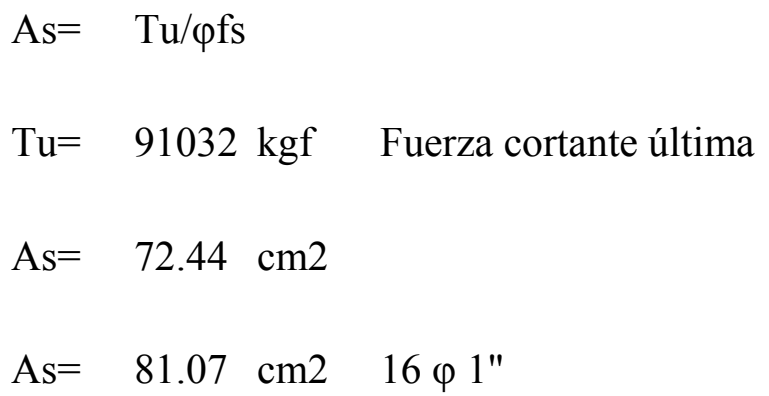


Espaciamiento en 2 capas de 1"

$\mathrm{S}(1 ")=\quad 15 \quad \mathrm{~cm}$

Entonces:

S (1")@15 cm

Diseño de armadura horizontal

$\mathrm{F} 22=62.669 \mathrm{tnf}$

$\mathrm{b}=1.00 \mathrm{~m}$

$\mathrm{f}^{\prime} \mathrm{c}=350 \mathrm{kgf} / \mathrm{cm} 2$

fy $=4200 \mathrm{kgf} / \mathrm{cm} 2$

$\mathrm{fs}=1400 \mathrm{kgf} / \mathrm{cm} 2 \quad$ Esfuerzo permisible del acero por fisuramiento (ACI 350 -06)

$\mathrm{fs}=0.33$ fy

$\mathrm{Ec}=15000 \cdot \sqrt{f^{\prime} c}$

$\mathrm{Ec}=280624 \quad \mathrm{kgf} / \mathrm{cm} 2$

Es $=2000000 \quad \mathrm{kgf} / \mathrm{cm} 2 \quad$ Módulo de elasticidad del acero

$\mathrm{n}=\mathrm{Es} / \mathrm{Ec} \rightarrow \quad 7.13$

$\varepsilon s h=0.0003 \quad$ Coeficiente de fisuramiento del concreto armado

$\mathrm{fct}=0.1 \mathrm{f}^{\prime} \mathrm{c} \quad$ Esfuerzo permisible del concreto por fisuramiento

$\mathrm{fct}=35$

$\mathrm{T}=61669 \mathrm{kgf} \quad$ Fuerza de tensión del elemento 
$\mathrm{t}=29.78 \mathrm{~cm} \quad$ Espesor del elemento

Cálculo del acero por tracción

$\mathrm{As}=\mathrm{Tu} / \varphi \mathrm{fs}$

$\mathrm{Tu}=62669 \mathrm{kgf} \quad$ Fuerza cortante última

$\mathrm{As}=49.74 \mathrm{~cm} 2$

$\mathrm{As}=50.67 \mathrm{~cm} 2 \quad 10 \varphi 1^{\prime \prime}$

Espaciamiento en 2 capas de 1"

$\mathrm{S}(1 ")=\quad 20 \quad \mathrm{~cm}$

Entonces:

S (1")@20 cm

\subsection{DISEÑO DE CIMENTACIÓN}

En este capítulo se diseña la cimentación tipo, la presente tesis da un alcance de la demanda que genera la estructura al suelo que luego se verificará la capacidad según con el Estudio de Mecánica de Suelos. Teniendo en consideración estos parámetros se calcularán las dimensiones de la cimentación. Para el análisis y diseño de esta estructura se toman en cuenta lo siguiente:

- Revisión por volteo

- Revisión por deslizamiento

- Revisión por capacidad portante. 


\subsubsection{Revisión por Volteo.}

La estabilidad por volteo se revisa por el momento producido por las fuerzas horizontales actuantes en la estructura, la cual se debe contrarrestar con los momentos resistentes del suelo, la cual no deberá ocasionar un volcamiento de la estructura.

El factor seguridad por volteo se expresa como:

$$
F S V=\frac{\sum M_{R}}{\sum M_{v}} \geq 1.50
$$

Donde:

$\sum \mathrm{M}_{\mathrm{R}}$ : Suma de momentos de las fuerzas que tienden a volcar la estructura respecto a la base.

$\sum \mathrm{M}_{\mathrm{v}}$ : Suma de momentos de las fuerzas que tienden a resistir el volteo respecto a la base,

\subsubsection{Revisión por Deslizamiento.}

Para la verificación por deslizamiento se debe tratar de equiparar las fuerzas horizontales externas y la fricción que se genera entre la estructura y el terreno de cimentación,

El factor por deslizamiento se expresa como:

$$
F S D=\frac{\sum F_{R}}{\sum F_{D}} \geq 1.50
$$

Donde:

$\sum F_{R}$ : Suma de fuerzas resistentes horizontales.

$\sum F_{D}$ : Suma de las fuerzas actuantes horizontales.

Los valores de coeficientes de fricción se asumen de acuerdo con suelos ideales, la cual serán empleados a partir del estudio de mecánica de suelos.

\subsubsection{Revisión por Carga de Suelo.}

La capacidad portante del suelo se debe plantear como un estudio previo al proyecto y a la construcción, por lo cual se debe hacer un muestreo de las capacidades geotécnicas del suelo 
cerca al área ocupada por la cimentación del tanque elevado. Teniendo en cuenta que la capacidad portante tendrá que ser mayor a la presión ejercida de la estructura a la cimentación.

$$
\delta_{M A X} \leq \delta_{A D M}
$$

Donde:

$\delta$ MAX: Presión máxima ejercida al suelo.

$\delta_{\text {ADM: }}$ Capacidad portante admisible del terreno de fundación. 


\section{CAPÍTULO IV: DISPOTISIVO ANTI-OLEAJE}

\subsection{DISPOSITIVO ANTI-SLOSHING PARA LA ESTRUCTURA}

Para el análisis teórico del tanque elevado se determinó un modelo matemático del sistema ideal la cual incluye propiedades físicas, estos parámetros son la rigidez $(\mathrm{K})$, la masa $(\mathrm{m})$, el amortiguamiento (C). Este último se halló en esta parte de la tesina para ver los efectos de amortiguamiento usando anillos deflectores que modifica el comportamiento de la masa del líquido en el tanque.

La vibración generada por el efecto del sismo genera en este tipo de estructuras un oleaje que se produce en la superficie libre del líquido. Este movimiento de la masa del fluido genera inestabilidades a la estructura y perjudica al correcto funcionamiento. Este movimiento del líquido que se genera debido a efectos sísmicos produce aceleraciones adicionales dentro de la estructura, esta aceleración del líquido dentro de la cuba posee infinitas frecuencias es por esto la forma de caracterizar un modelo teórico entre el fluido y la estructura será de acuerdo a su rigidez, masa y su amortiguamiento. Así mismo, con este modelo que incluye el amortiguamiento de anillos deflectores se pretende minimizar la influencia del oleaje en la estructura. ${ }^{30}$

\subsection{PARAMETROS}

Altura máxima de oleaje

La aceleración horizontal hace que el líquido almacenado comience a moverse en la superficie generando oleaje (sloshing), esto genera un desplazamiento vertical el cual se calcula con lo siguiente:

$\mathrm{D}=18.4$

$$
d_{\text {máx }}=\left(\frac{D}{2}\right) \cdot\left(Z S U \cdot C_{C}\right)
$$

${ }^{30}$ Cfr: Bruno, Scarabino, Bacchi 2012: 47 


$$
\begin{aligned}
& \mathrm{Z}=0.45 \\
& \mathrm{~S}=1 \\
& \mathrm{U}=1.5 \\
& \mathrm{Cc}=0.270119328 \\
& \text { dmáx }=1.68 \mathrm{~m}
\end{aligned}
$$

Amortiguamiento del muro

$$
\zeta=C \cdot v^{\frac{1}{2}} \cdot a^{-\frac{3}{4}} \cdot g^{-\frac{1}{4}}
$$

Donde:

a: Radio de la cuba

g: Aceleración de la gravedad

v: Velocidad cinemática del agua

h: Altura de la cuba

C2: Coeficiente para el amortiguamiento en el tanque

Tabla 30-IV: Valores de Coeficiente Numérico "C" para la Estimación de Amortiguamiento Viscoso en Varios Tanques

\begin{tabular}{|c|c|c|}
\hline Forma Tanque & Coeficiente C & $\mathrm{h} / \mathrm{a}$ \\
\hline Cilíndrico (Fondo Plano) & 0.56 & $>1$ \\
\hline Cilíndrico (Fondo Plano) & 0.79 & $>1$ \\
\hline Cilíndrico (Fondo Plano) & 0.86 & $>1$ \\
\hline Cilíndrico (Fondo Esférico) & $0.79 \mathrm{xC} 2$ & TODOS \\
\hline Esfera & $0.79 \times \mathrm{xC} 3$ & TODOS \\
\hline Cónico & $0.79 \times \mathrm{xC}^{\wedge}-1 / 2 \times \mathrm{xC} 5$ & -- \\
\hline
\end{tabular}

Fuente: "Slosh Suppression”; Nasa Space Vehicle Design Criteria; p15 
$\mathrm{g}=9.81 \mathrm{~m} / \mathrm{s} 2$

$\mathrm{v}=1.139 \times 10 \mathrm{E}-6 \mathrm{~m} 2 / \mathrm{s}$

$\mathrm{h}=\quad 7.4 \mathrm{~m}$

$\mathrm{h} / \mathrm{a}=0.80$

$\mathrm{C}=\quad 0.95$

$\mathrm{C} 2=1.2$

$\zeta=\quad 0.00011$ (ratio de amortiguamiento)

Figura 39 - IV: cálculo del coeficiente C2 en función de la altura del tanque y el radio.

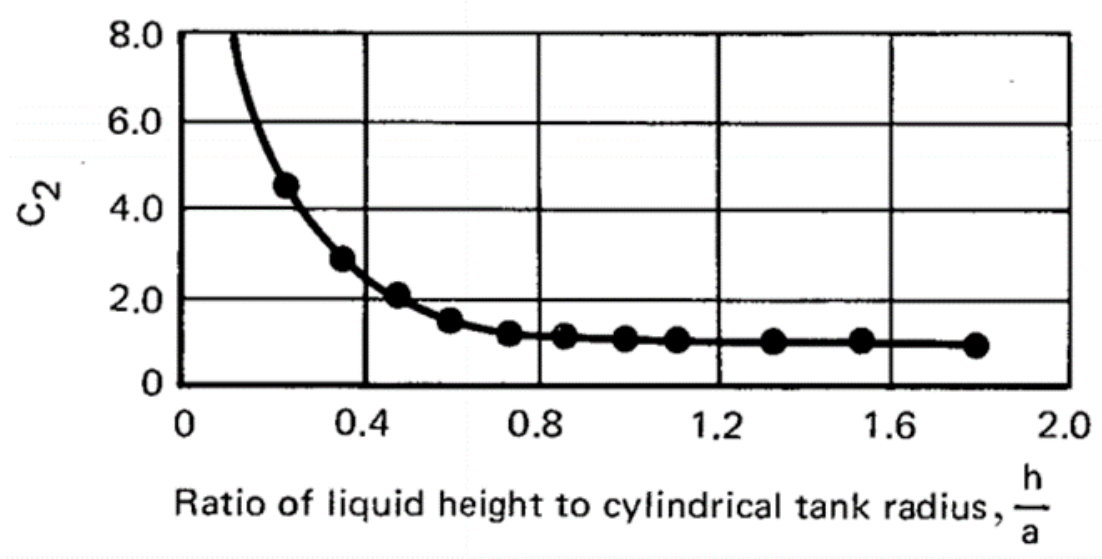

Fuente: "Slosh Suppression"Abramson H. p 16

Anillos Deflectores

Se usarán 8 anillos deflectores en la cuba.

$$
\zeta=\frac{\delta}{2 \pi}=2.83 e^{-4 \cdot 6 \cdot \frac{d}{a}} \cdot\left(\frac{2 W}{a}-\left(\frac{W}{a}\right)^{2}\right)^{\frac{3}{2}} \cdot\left(\frac{n}{a}\right)^{1 / 2}
$$

Donde:

$\mathrm{d}=\quad 6.4 \mathrm{~m}$ (Profundidad de la cuba) 

$\mathrm{w}=\quad 2.50 \mathrm{~m}$ (Profundidad de deflectores)
$\mathrm{n}=\quad 1.68 \mathrm{~m}$ (altura máxima del oleaje)
$\delta=\quad$ factor de amortiguamiento
$\mathrm{a}=\quad 9.2 \mathrm{~m}$ (Radio de tanque)
$\zeta=0.1107$

$$
\zeta=\frac{\delta}{2 \pi}
$$

$$
\delta=0.696
$$

Figura 40-IV: Modelo de rigideces y deflectores que influyen en la masa del fluido

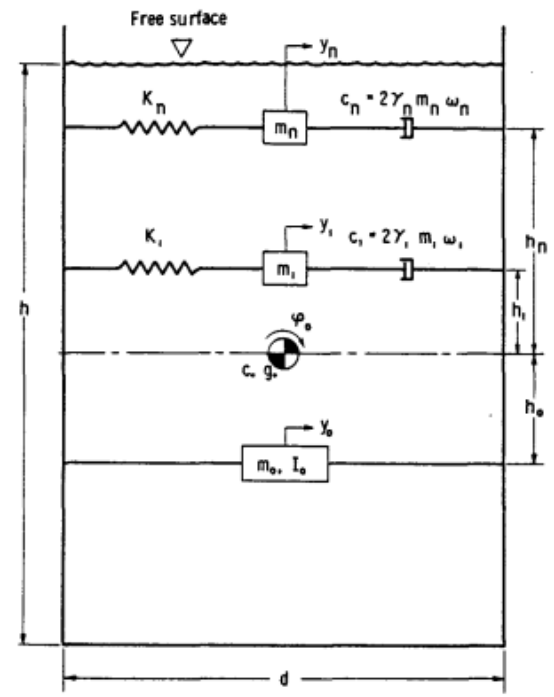

Fuente: "The Dynamic Behavior of Liquids in Moving Containers" Abramson H. p215

$$
C=2 \cdot \gamma \cdot m \cdot \omega
$$

Donde:

$\gamma$ : Factor de amortiguamiento

$\omega:$ Frecuencia de la masa convectiva

$\mathrm{C}=228.5433326$ 


$$
\omega^{2}=k_{2} / m_{2}
$$

$\omega=\quad 1.58 \quad$ Amortiguador Dinámico

$\mathrm{k}=\quad 259.74 \mathrm{Tn} / \mathrm{m}$ Rigidez Convectiva

$\mathrm{m}=\quad 103.92$ Tn.f.s^${ }^{\wedge} / \mathrm{m} \quad$ Masa Convectiva

En la figura 44-IV se muestran los anillos deflectores distribuidos usados para el análisis dinámico.

Figura 41-IV: Anillos deflectores en la cuba.

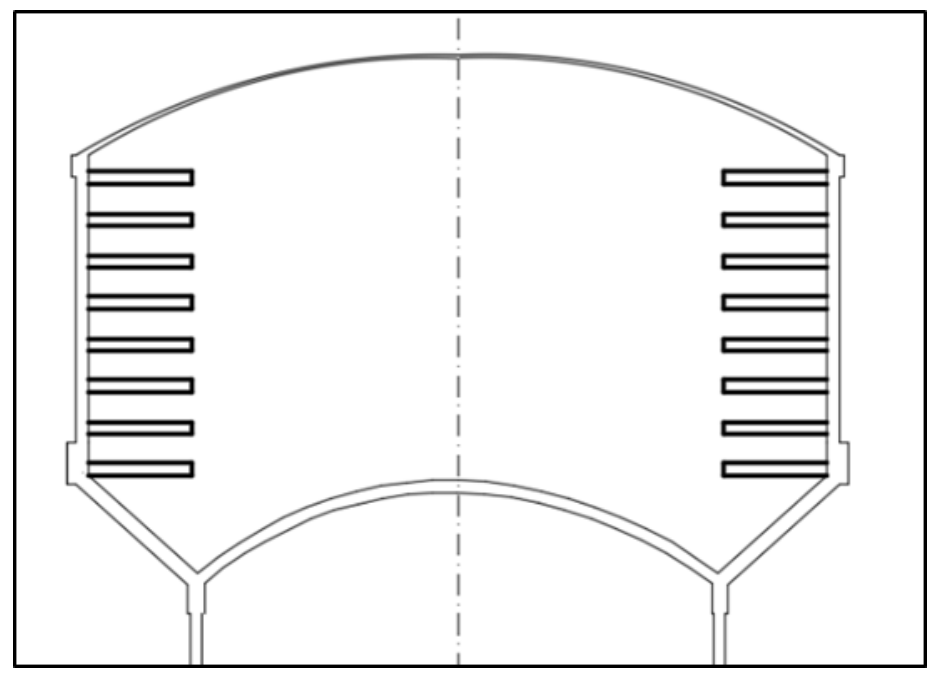

Fuente: Elaboración Propia 


\section{CAPÍTULO V: ANÁLISIS DE RESULTADOS}

\subsection{ANÁLISIS DE RESULTADOS CON EL USO DE ANILLOS DEFLECTORES}

A continuación, se muestra un resumen de los resultados obtenidos con el uso de anillos deflectores en el programa computacional SAP2000, los resultados serán comparados con los resultados del análisis dinámico sin el uso de los anillos deflectores para mostrar el impacto que genera el uso del sistema de anillos deflectores en la estructura.

\subsubsection{COMPARACIÓN DE FUERZAS ACTUANTES EN LA ESTRUCTURA}

Los elementos sometidos directamente a la influencia del líquido son: la cuba, el fondo troncocónico y la viga inferior. En la siguiente tabla se muestran las fuerzas obtenidas del análisis dinámico.

Tabla 31-IV: Comparación de Fuerzas Actuantes en la Estructura

\begin{tabular}{|c|c|c|c|c|}
\hline \multirow{4}{*}{ Elemento } & Fuerzas & $\begin{array}{c}\text { SIN } \\
\text { DEFLECTORES }\end{array}$ & $\begin{array}{c}\text { CON } \\
\text { DEFLECTORES }\end{array}$ & \% Reducido \\
\hline \multirow{4}{*}{$\begin{array}{c}\text { FONDO } \\
\text { TRONCOCÓNICO- } \\
\text { VIGA INFERIOR }\end{array}$} & F1 (Tn) & 103.63 & 67.94 & $34 \%$ \\
\cline { 2 - 5 } & F2 (Tn) & 173.16 & 113.12 & $35 \%$ \\
\cline { 2 - 5 } & M1 (Tn-m) & 97.09 & 65.17 & $33 \%$ \\
\cline { 2 - 5 } & M3 (Tn-m) & 58.44 & 39.18 & $33 \%$ \\
\cline { 2 - 5 } & V13 (Tn-m) & 15.23 & 9.98 & $34 \%$ \\
\cline { 2 - 5 } & V23 (Tn/m) & 11.10 & 7.53 & $32 \%$ \\
\hline \multirow{5}{*}{ CUBA } & F1 (Tn) & 108.70 & 13.27 & $34 \%$ \\
\cline { 2 - 5 } & F2 (Tn) & 184.37 & 10.31 & $35 \%$ \\
\cline { 2 - 5 } & M1 (Tn-m) & 24.01 & 15.27 & $35 \%$ \\
\cline { 2 - 5 } & M2 (Tn-m) & 14.36 & 9.40 & $35 \%$ \\
\cline { 2 - 5 } & M3 (Tn-m) & 8.84 & 5.73 & $35 \%$ \\
\cline { 2 - 5 } & V13 (Tn-m) & 3.52 & 2.29 & $35 \%$ \\
\cline { 2 - 5 } & V23 (Tn/m) & 8.93 & 6.09 & $32 \%$ \\
\hline
\end{tabular}

Fuente: Elaboración propia de resultados obtenidos del programa computacional SAP2000. 
Como se aprecia en la tabla 29-IV con el uso de anillos deflectores se reducen en un porcentaje de alrededor del 30\% las fuerzas que actúan en los elementos estructurales contenedoras de la masa del agua. Los anillos deflectores actúan como amortiguadores para reducir los efectos de la masa convectiva en el tanque.

\subsubsection{COMPARACÍON DE DESPLAZAMIENTOS}

Se realizó el modelamiento de la estructura con el uso de anillos deflectores, los cuales se modelaron como amortiguadores, en el programa computacional SAP2000. Del modelamiento se obtuvieron los desplazamientos. En la siguiente tabla, se comparan los resultados de desplazamientos del análisis dinámico del tanque.

Tabla 32-IV: Comparación de desplazamientos para el análisis dinámico.

\begin{tabular}{|c|c|c|c|}
\hline NIVEL & hi(m) & $\begin{array}{c}\text { Desplazamiento sin } \\
\text { anillos deflectores }\end{array}$ & $\begin{array}{c}\text { Desplazamiento con } \\
\text { anillos deflectores }\end{array}$ \\
\hline VIGA SUP+CUP.SUP & 31.5 & 0.0272 & 0.0197 \\
\hline
\end{tabular}

Fuente: Elaboración propia de resultados obtenidos del programa computacional SAP2000.

De la tabla se concluye que el uso de los amortiguadores reduce el impacto de la masa convectiva, y esto se traduce también en una reducción en los desplazamientos para el análisis dinámico de la estructura. 


\section{CONCLUSIONES}

Se alcanzó un $93.38 \%$ de masa participativa la cual cumple con la norma vigente E 030 que exige valores mayores al 90\% de masa participativa.

Los valores de las cortantes basales dinámica y estática fueron de 1497 Ton y 1660 Ton, respectivamente. De estos valores se obtiene una relación que cumple con $90 \%$ Vest $<$ Vdin cumpliendo de esta manera con lo establecido por la norma E 030.

En el espectro usado para el análisis dinámico, se tomaron en cuenta dos coeficientes de respuesta sísmica $(\mathrm{Ci}$ y $\mathrm{Cc}$ ). El Ci para periodos cortos $(<1 \mathrm{seg})$ y el $\mathrm{Cc}$ para periodos largos ( $>1 \mathrm{seg}$ ), este espectro se adecua este tipo de análisis ya que en el tanque actúan dos tipos de masas, la convectiva e impulsiva.

Se comprobó que el periodo hallado manualmente $(\mathrm{T}=3.98 \mathrm{~s})$ y del del programa SAP2000 $(\mathrm{T}=4.01 \mathrm{~s})$ son semejantes.

La norma peruana no contempla el factor de reducción para este tipo de estructuras, por lo cual se usó el factor de reducción de la norma ACI 350 para tanques elevados, donde se define un valor de $\mathrm{R}=2$ para la masa impulsiva y $\mathrm{R}=1$ para la masa convectiva.

El esfuerzo en las paredes del tanque elevado es menor que el esfuerzo de Von Mises 36.920 $\mathrm{kg} / \mathrm{cm} 2<37.416 \mathrm{~kg} / \mathrm{cm} 2$, la cual indica que el material no fallará de manera frágil.

Se propuso la implementación de anillos deflectores para reducir la incidencia de la masa convectiva en la estructura. La cual genera una reducción de las fuerzas y desplazamientos en la estructura de un $30 \%$ y $20 \%$ respectivamente. 


\section{RECOMENDACIONES}

La norma peruana no contempla factores ni parámetros necesarios para los correctos análisis del tanque elevado, debido a esto se recomienda la implementación de una norma para el diseño de este tipo de estructura no convencionales.

Sería ideal poder simular en laboratorio sísmico y con modelos a escala de tanques elevados para observar la interacción del líquido con la estructura. Es fundamental profundizar el conocimiento para así asegurar la integridad este tipo de estructuras importantes ante un evento sísmico.

Esta investigación aporta una metodología para el análisis y diseño estructural de un tanque elevado de gran capacidad, la cual se deberá complementar empleando los factores dictados por el ACI y ASCE.

Profundizar las investigaciones acerca de dispositivos anti - sloshing, ya que como se ha demostrado mediante la modelación computacional disminuye los esfuerzos y desplazamientos en la estructura. 


\section{LINEAS FUTURAS}

En lo que concierne a las líneas de investigación futura, durante el proceso de elaboración de este trabajo se ha considerado interesantes los temas que se exponen a continuación:

Resultaría muy útil profundizar el estudio del modelo de G.W. Housner para diferentes tipos tanques en las que se puedan utilizare dispositivos anti - sloshing los cuales se pueden idealizar como amortiguadores, ya que esto nos ayuda a disminuir los esfuerzos que se producen en la estructura.

Es importante incluir normas para nuestra realidad con factores y parámetros necesarios para el adecuado diseño de tanques elevados. 


\section{BIBLIOGRAFIA}

Abramson, H. (1969). SLOSH SUPRESSION. abril 12, 2016, de National Aeronautics and Space Administration Sitio web: http://www.dept.aoe.vt.edu/ cdhall/courses/aoe4065/NASADesignSPs/sp8031.pdf

Abramson H. . (1966). The Dynamic Behavior of Liquids in Moving Containers. 5 de abril, 2016, de Institute for the National Aeronautics and Space Administration Sitio web: http://ntrs.nasa.gov/archive/nasa/casi.ntrs.nasa.gov/19670006555.pdf

ACI (2009) Guide for the Analysis, design, and Construction of Elevated Concrete and Composite Steel-Concrete Water Storage Tanks. 371R-08. USA: ACI

ACI Committee 350, “Code Requirements for Environmental Engineering Concrete Structures (ACI 350-01) and Commentary (350R-01),” American Concrete Institute,

Farmington Hills, Mich., 2001.

ASCE. (2005). Minimun Design Loads for Buildings and Other Structures. Reston, Virginia: American Society of Civil Engineers.

Bruno, L.; Scarabino, A.; Bacchi, F. (diciembre 20, 2012). DISEÑO DE DISPOSITIVOS ANTISLOSHING PARA UN VEHICULO ESPACIAL DE COMBUSTIBLE LÍQUIDO. mayo 10, 2016, de Grupo de Fluidodinámica computacional Sitio web: http://anales.fisica.org.ar/journal/index.php/analesafa/article/download/1437/1622

Chacaltana F. Franco, A. Reyes, E.. (2011). Diseño de Reservorio Elevado tipo Fuste. Lima-Perú: Universidad de Ciencias Aplicas (UPC). CRESPO María, RUBINSTEIN Marcelo, CORESTEIN Georgina. (26/08/2015).

Compagnoni M.; Curadelli R. \& Martinez C. (2012). ANÁLISIS DEL COMPORTAMIENTO DINÁMICO DE TANQUES CILÍNDRICOS BAJO EXCITACIÓN SÍSMICA. En Mecánica Computacional Vol XXXI (2219-2230). Salta, Argentina: Asociación Argentina de Mecánica Computacional.

Compagnoni M, Curadelli R., Martinez C. (2013): Evaluación de Modelos de Tanques Rectangulares Contenedores de Líquido Bajo Excitación Sísmica.

(http://www.cimec.org.ar/ojs/index.php/mc/article/viewFile/4467/4397)

(Consulta: 14 de octubre del 2015).

Computers \& Structures, INC.. (2015). CSI Analysis Reference Manual CSI. (www.csiamerica.com)

(Consulta: 06 de Junio del 2016).

De Castro C. (2013). Teorías de Falla Bajo Cargas Estáticas. abril 10, 2016, de Universidad de Salamanca Sitio web: http://dim.usal.es/eps/im/roberto/cmm/Teorasdefallabajocargasestticas.pdf 
Flores, V. (2009): Comportamiento Mecánico de Tanques Atmosféricos de Almacenamiento. (http://tesis.ipn.mx/handle/123456789/5900?show=full)

(Consulta: 07 de Octubre del 2015).

Hickey, H. (2008). Water Supply Systems and Evaluation Methods. U.S: FEMA.

Huamaní, H.. (2015). Diseño estructural de un reservorio elevado tipo fueste de concreto armado. Lima, Perú: Universidad Nacional de Ingeniería.

Jim Orihuela Canchari1, Natividad A. Sánchez Arévalo2. (2016, Diciembre 4). DISEÑO ESTRUCTURAL DE TANQUES RECTANGULARES Y SUS APLICACIONES. Revista Ingenium, 1,13

LIVAOGLU Y DOGANGÜN (2006): Simplified seismic analysis procedures for elevated tanks considering fluid-structure-soil interaction.

(http://www.sciencedirect.com/science/article/pii/S0889974606000041)

(Consulta: 14 de Octubre del 2015).

Martinez E.; Sanchez L. (Abril 2004). Análisis Sísmico de tanques elevador. Tesis, 1, 11 - 30. 26 de Abril 2015, De Repositorio UIS Base de datos.

Munshi, J. (2002). Design of Liquid-Containing Concrete Structures For Earthquake Forces. USA: Construction Technology Laboratories, Inc.

Nieto, C. \& Zhañay, W.. (2011). Cargas de Diseño. En DISEÑO DE UN TANQUE APOYADO DE FERROCEMENTO PARA LA COMUNIDAD DE SANTA ROSA DE CHICHIN, PERTENECIENTE A LA PARROQUIA JADÁN DEL CANTÓN GUALACEO (pp. 19-24). Cuenca-Ecuador: UNIVERSIDAD DE CUENCA.

Organización Panamericana de Salud. (2005). GUÍAS PARA EL DISEÑO DE RESERVORIOS ELEVADOS DE AGUA POTABLE. Octubre 10, 2015, de COSUDE Sitio web: http://www.bvsde.opsoms.org/tecapro/documentos/agua/160esp-disenoreservorioselevados.pdf

REGLAMENTO NACIONAL DE EDIFICACIONES (2006). Norma Técnica Peruana Vigente E 020 Cargas.

REGLAMENTO NACIONAL DE EDIFICACIONES (2016). Norma Técnica Peruana Vigente E 030 Diseño Sismoresistente.

REGLAMENTO NACIONAL DE EDIFICACIONES (2009). Norma Técnica Peruana Vigente E 060 Concreto Armado.

Rivera, J. (1986). Análisis Sísmico de Reservorios Elevados con Estructura Cilíndrica de Soporte. Lima-Perú: Universidad Nacional de ingeniería.

Rivera, J.. (06/2000). DISEÑO ESTRUCTURAL DE OBRAS HIDRÁULICAS. EL INGENIERO CIVIL, 1, 5-14. 
Tavera, H.,Bernal, I.,\& Salas,H.. (2007). El Sismo de Pisco del 15 de Agosto, 2007 (7.9Mw). Julio 18, 2016, de INSTITUTO GEOFISICO DEL PERU Sitio web: https://www.eeri.org/wpcontent/uploads/Pisco150807.pdf

Universidad de Alicante. "SISMOLOGIA E INGENIERIA SISMICA". Universidad de Alicante,

Otazzi, G. (2004): Material de Apoyo para la Enseñanza de los Cursos de Diseño y Comportamiento del Concreto Armado.

(http://tesis.pucp.edu.pe/repositorio/bitstream/handle/123456789/1055/OTTAZZI_PASINO_

GIANFRANCO_MATERIAL_ENSE\%C3\%91ANZA_CONCRETO_ARMADO.pdf?sequen $\mathrm{ce}=1)$

(Consulta: 27 de abril de 2016) 